\title{
Hydrogen Fuel Cells and Storage Technology: Fundamental Research for Optimization of Hydrogen Storage and Utilization
}

\author{
"An R\&D project designed to combine theory \\ and experiment to address specific fundamental aspects \\ of hydrogen storage and utilization“
}

March 29, 2011

Funded by the U.S. Department of Energy Solar Technologies Program

DE-FG36-05G085028

Award Recipient:

UNLV RESEARCH FOUNDATION

8311 W. Sunset Road, Suite 200

Las Vegas, NV 89113

702-895-2833

loni.benard@unlv.edu 


\section{Final Report}

Project Title:

Project Period:

Date of Report:

Recipient:

Award Number:

Working Partner:

Cost-Sharing Partners: UNLV

DOE Manager:

Collaborators:

Technical Contact:

Dr. Robert F.D. Perret

(702) 630-1542

ntsllc@rperret.com

March 29, 2011

DE-FG36-05GO85028
Hydrogen Fuel Cells and Storage Technology: Fundamental Research for Optimization of Hydrogen Storage and Utilization

September 15, 2005 to December 31, 2010

UNLV Research Foundation

Jesse Adams, DOE-GO Project Manager

UTC Power, Inc., Windsor, CT

Rice University, Houston, TX

Lawrence Livermore National Laboratory, Livermore, CA

Lawrence Berkeley National Laboratory, Berkeley, CA

Principal Investigators: Clemens Heske, UNLV

Balakrishnan Nudavalath, UNLV

Oliver Hemmers, UNLV

Andrew L. Cornelius, UNLV

David W. Hatchett, UNLV

Chulsung Bae, UNLV

Tao Pang, UNLV

Eunja Kim, UNLV

Co-PIs:

D. Lindle, UNLV

W. Stolte, UNLV

K. Lipinska-Kalita, UNLV 


\section{TABLE OF CONTENTS}

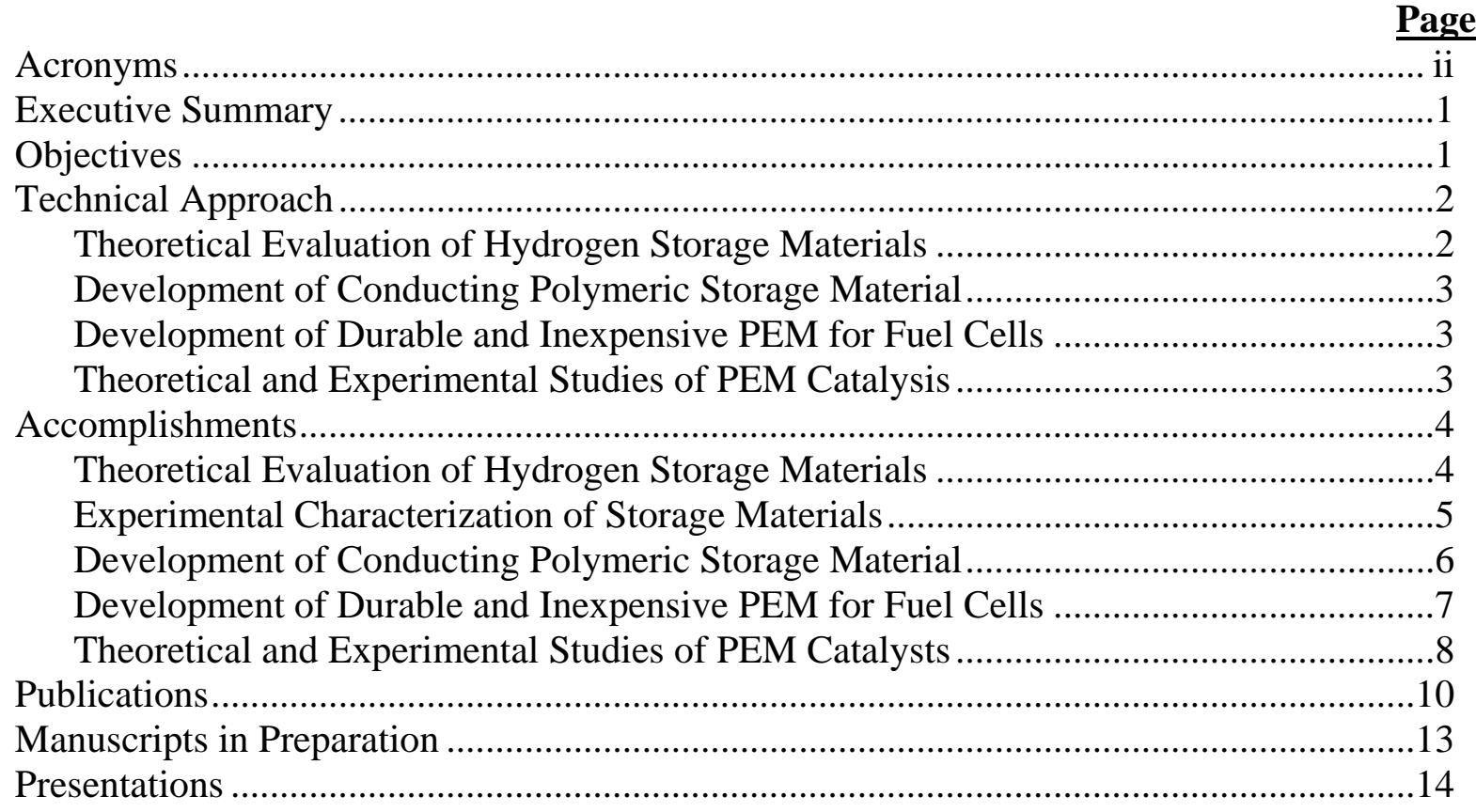

\section{ATTACHMENTS}

A Local Spectroscopy of Nanomaterials for Hydrogen Storage (C. Heske) ................19

B X-Ray Spectroscopy Studies for Hydrogen Storage - Study of Interactions in the System: Carbon Nanotubes - Transition Metals Hydrogen” (O. Hemmers, D. Lindle, W. Stolte, K. Lipinska-Kalita).......................63

C Metal Hydrides (A. Cornelius) .....................................................................72

D Chemical Synthesis Using either Pd(iv) or Pd(ii) and the Monomer/ Oligomer, Electrochemical Synthesis of PANI followed by Reduction of Pd(iv) or Pd(ii) (D. Hatchett) ........................................................................ 73

E Hydrogen Storage and Fuel Cell Membranes (B. Naduvalath)..............................76

F Atomic and Electronic Structure of Carbon-Based Nanomaterials for Hydrogen-Storage Application (C. Bae) ......................................................... 81

G Quantum Monte Carlo Simulator of Hydrogen in Host Matrix (T. Pang) ................87

H Carbon 3D-Nanoframework, Single-Wall Carbon Nonotubes (E. Kim) ..................88 


\section{ACRONYMS}

$\begin{array}{ll}\text { ALS } & \text { Advanced Light Source } \\ \text { DFT } & \text { Density Functional Theory } \\ \text { GGA } & \text { Generalized Gradient Approximation } \\ \text { IEC } & \text { Ion Exchange Capacity } \\ \text { FCAST } & \text { Hydrogen Fuel Cells and Storage Technology } \\ \text { LBNL } & \text { Lawrence Berkeley National Laboratory } \\ \text { LDA } & \text { Local Density Approximation } \\ \text { LLNL } & \text { Lawrence Livermore National Laboratory } \\ \text { MD } & \text { Molecular Dynamics } \\ \text { PANI } & \text { Polyaniline } \\ \text { PEM } & \text { Proton Exchange Membrane } \\ \text { SEM } & \text { Scanning Electron Microscopy } \\ \text { STA } & \text { Sulfonated Terephthalic Acid } \\ \text { STS } & \text { Scanning Tunneling Spectroscopy } \\ \text { SWCNT } & \text { Single Wall Carbon Nanotube } \\ \text { SWRI } & \text { Southwest Research Institute } \\ \text { TA } & \text { Terephthalic Acid } \\ \text { UNLV } & \text { University of Nevada, Las Vegas } \\ \text { UPS } & \text { Ultraviolet Photoelectron Spectroscopy } \\ \text { UTC } & \text { United Technologies Corporation } \\ \text { XAES } & \text { X-Ray Auger Electron Spectroscopy } \\ \text { XAS } & \text { X-Ray Absorption Spectroscopy } \\ \text { XES } & \text { X-Ray Emission Spectroscopy } \\ \text { XPS } & \text { X-Ray Photoelectron Spectroscopy } \\ \end{array}$




\section{EXECUTIVE SUMMARY}

Design and development of improved low-cost hydrogen fuel cell catalytic materials and high-capacity hydrogenn storage media are paramount to enabling the hydrogen economy. Presently, effective and durable catalysts are mostly precious metals in pure or alloyed form and their high cost inhibits fuel cell applications. Similarly, materials that meet on-board hydrogen storage targets within total mass and volumetric constraints are yet to be found. Both hydrogen storage performance and cost-effective fuel cell designs are intimately linked to the electronic structure, morphology and cost of the chosen materials. The FCAST Project combined theoretical and experimental studies of electronic structure, chemical bonding, and hydrogen adsorption/desorption characteristics of a number of different nanomaterials and metal clusters to develop better fundamental understanding of hydrogen storage in solid state matrices.

Additional experimental studies quantified the hydrogen storage properties of synthesized polyaniline(PANI)/Pd composites. Such conducting polymers are especially interesting because of their high intrinsic electron density and the ability to dope the materials with protons, anions, and metal species. Earlier work produced contradictory results: one study reported $7 \%$ to $8 \%$ hydrogen uptake while a second study reported zero hydrogen uptake.

Cost and durability of fuel cell systems are crucial factors in their affordability. Limits on operating temperature, loss of catalytic reactivity and degradation of proton exchange membranes are factors that affect system durability and contribute to operational costs. More cost effective fuel cell components were sought through studies of the physical and chemical nature of catalyst performance, characterization of oxidation and reduction processes on system surfaces. Additional development effort resulted in a new hydrocarbon-based high-performance sulfonated proton exchange membrane (PEM) that can be manufactured at low cost and accompanied by improved mechanical and thermal stability.

\section{OBJECTIVES}

1. Coordinate theory and experiment to address specific fundamental aspects of hydrogen storage and utilization.

2. Quantify:

a. Electronic structure, density of states and pseudo-potential functions for defined storage media,

b. The mechanisms of hydrogen adsorption/desorption in potential storage materials,

c. Catalysis of hydrogen adsorption and dissociation on platinum/platinum alloy surfaces. 
3. Optimize:

a. Specific capacity of carbon and boron-nitride storage matrices of different structures and compositions,

b. Operating temperatures and durability of hydrogen fuel cell catalytic surfaces.

2. Demonstrate specific capacity storage performance and catalytic surface degradation mechanisms for selected configured materials.

\section{TECHNICAL APPROACH}

\section{Theoretical Evaluation of Hydrogen Storage Materials}

Highly fundamental studies of hydrogen confinement and equation-of-state of enriched hydrogen compounds were accompanied by development of atomic and molecular configurations of selected material compositions specifically to provide a basis for calculating hydrogen storage reaction barriers and energies of hydrogen adsorption and dissociation. Density functional theory (DFT) calculations were applied to quantify configuration stability and hydrogen association and dissociation with selected configurations was evaluated. Carbon nanotubes and fullerenes were assessed as reference materials for exploring the effects of incorporating enhancing impurities like $\mathrm{Ti}$ and $\mathrm{Li}$. More complex nanostructures were conceived and energetics of hydrogen adsorption and desorption were explored computationally.

\section{Experimental Characterization of Storage Materials}

An atomic hydrogen source was constructed to provide hydrogenated samples of carbon nanotubes and Fullerenes to characterize the electronic structures of the starting materials and changes accompanying hydrogenation. Capabilities at the University of Nevada, Las Vegas (UNLV) and at the Advanced Light Source (ALS) at the Lawrence Berkeley National Laboratory (LBNL) were marshaled to provide X-ray Emission Spectroscopy (XES), X-ray Absorption Spectroscopy (XAS), X-ray Photoelectron Spectroscopy (XPS), X-ray-excited Auger Electron Spectroscopy (XAES), Ultraviolet-excited Photoelectron Spectroscopy (UPS), Scanning Electron Microscopy (SEM) and Scanning Tunneling Spectroscopy (STS) of synthesized samples to quantify morphology and electronic structure of samples. Deposition of metals ( $\mathrm{Ti}$ and $\mathrm{Li}$ ) on carbon nanomaterials (SWCNTs and $\mathrm{C}_{60}$ ) under ultra-high-vacuum conditions was undertaken and the chemical and electronic structures were evaluated using laterally area-integrating spectroscopic techniques (XPS, XAES, and UPS). These capabilities were used both to validate equations of state of hydrogen-enriched compounds and to understand the impact of the metal deposits on the surface of carbon nanostructures and the oxidation behavior of the metals and the consequences for their application in hydrogen storage. 


\section{Development of Conducting Polymeric Storage Material}

Earlier contradictory reports of conductive polymer hydrogen storage effectiveness were addressed by synthesizing and characterizing Pd-incorporated polyanilin polymers. Two synthesis routes were explored:

1. Chemical synthesis using either Pd(iv) or Pd(ii) and the monomer/oligomer.

2. Electrochemical synthesis of PANI followed by reduction of $\mathrm{Pd}(\mathrm{iv})$ or $\mathrm{Pd}(\mathrm{ii})$.

Hydrogen storage properties of the materials and independent measurement of the hydrogen sorption of the most promising material was obtained.

\section{Development of Durable and Inexpensive PEM for Fuel Cells}

Aromatic polyamides are known to have excellent chemical stability and mechanical properties that are critical features for an effective polymer electrolyte membrane (PEM) in fuel cell applications. Initially prepared sulfonated aromatic poly(ether amide)s showed promising proton conductivity, comparable to that of Nafion at $80{ }^{\circ} \mathrm{C}$ and $100 \%$ relative humidity, but poor stability was found when ion exchange capacity (IEC) was increased to improved the proton conductivity further. To improve proton conductivity of the membrane further without excessive water uptake, synthesis techniques were developed to incorporate fluorinated moiety along the polymer chains.

\section{Theoretical and Experimental Studies of PEM Catalysis}

Pt-based fuel cell catalysts were prepared at UTC Power, Inc. and at UNLV. The samples were measured using synchrotron- and lab-based soft x-ray and UV spectroscopies to understand their chemical properties, to investigate the presence (or absence) of a core-shell structure, and to gain insight into their electronic properties.

First principles calculations of the electronic structure of small clusters of Pt-Co alloy were performed to understand the effect of adsorption of $\mathrm{H}_{2}, \mathrm{O}_{2}$ and $\mathrm{CO}$ molecules on different binding sites. Sites like Co bonds, Pt bonds, joint Co-Pt bonds, Pt-Pt bond and Pt-Co-Pt face-bonding on an optimized tetrahedron $\mathrm{Pt}_{3} \mathrm{Co}_{\mathrm{C}}$ cluster have been studied. Additional work addressed the role of substrate in cluster configuration and favored bonding mechanisms. 


\section{ACCOMPLISHMENTS}

Results and accomplishments will be summarized below in the five categories described above. Please refer to the Appendices for more detailed information.

\section{Theoretical Evaluation of Hydrogen Storage Materials}

Fundamental quantum Monte Carlo simulations of hydrogen in a host matrix were completed. Spheroidal models of the host matrix were modeled and simulations performed for a single spheroid configuration and a "double-sphere" configuration with volume constrained to that of the single optimized spheroid with the same bond length. The results showed that a hydrogen molecule has higher energy in a double-sphere box suggesting that a hydrogen molecule fixed in a matrix with a cavity similar to a doublesphere box is more readily recovered. Further work would be required to apply these simulations to configured specific storage matrices. It could be speculated that matrix confinement parameters could be designed with incorporated impurities to meet hydrogen extraction/recovery targets while retaining matrix integrity. Details of these calculations can be found in scientific publications cited at the end of this report.

Based on first-principles calculations, a novel class of three-dimensional storage materials were conceived that consisted of small diameter single-walled carbon nanotubes (SWCNTs) functionalized by organic ligands. Density functional theory calculations were used to determine the stable structures and properties of nanoframeworks consisting of $(5,0)$ and $(3,3)$ SWCNTs constrained by phenyl spacers. Valence and conduction properties, as well as normal modes, of pristine nanotubes were found to change significantly upon functionalization. These changes could serve to demonstrate successful synthesis of the proposed framework structures. $A b$ initio molecular dynamics simulations showed that such systems are thermodynamically stable for hydrogen storage. It is anticipated that synthesis techniques could be developed to produce functionalized carbon nanotubes for purposes of validation and further modification for improved performance.

Hydrogen storage efficacy was studied for model organometallic compounds consisting of $\mathrm{Sc}, \mathrm{Ti}$, and $\mathrm{V}$ transition metal atoms bound to $\mathrm{C}_{m} \mathrm{H}_{m}$ rings $(m=4-6)$ using density functional theory (DFT). This work was published in J. Chem. Phys. (2007) and it has so far been cited 22 times, including Physical Review Letters and Applied Physics Letters. A related work performed in collaborations with Prof. Boris Yakobson at Rice University and Dr. Hansong Cheng at Airproducts and Chemicals, Inc., proposed a novel class of 3D materials consisting of ultra-small diameter single-walled carbon nanotubes covalently functionalized by organic ligands as potential hydrogen storage media. Ab initio CarParrinello molecular dynamics (MD) simulations showed that the proposed nanoframeworks are energetically and thermodynamically stable up to at least $600 \mathrm{~K}$. The proposed structure, published in Chem. Phys. Lett. (2007), was experimentally validated in a recent work published by Tour and coworkers at Rice university and NREL (Leonard et al., J. Am. Chem. Soc. 131, 723 (2009)). They demonstrated the utility of similar nanoengineered carbon scaffolds for hydrogen storage. Based on initial measurements, nanoscaffolds with surface areas of $2000 \mathrm{~m}^{2} / \mathrm{g}$ are capable of a hydrogen 
uptake of $7.4 \mathrm{wt} \%$ at 2 bar. A related study investigated the successive adsorption of hydrogen atoms on boron nitride graphitic sheets. This work showed that hydrogen adsorption on $\mathrm{BN}$ surfaces is stabilized by the formation of contiguous $\mathrm{H}-\mathrm{H}$ orthodimer structures. Results of this work was featured on the cover of Phys. Chem. Chem. Phys. \{Phys. Chem. Chem. Phys., 10, 5184-5187 (2008)\}. A collaborative work with FCAST team member and organic chemistry colleague Dr. Chulsung Bae investigated the mechanism of proton transport in new polymer-electrolyte membranes based on sulfonated aromatic polyarylenes synthesized by Dr. Bae's group for high temperature fuel cell applications. This work (purely computational) was featured on the cover of $\mathrm{J}$. Phys. Chem. B. \{J. Phys. Chem. B 112, 3283-3286 (2008)\}.

Experimental observations combined with $a b$ initio calculations were used to study reversible hydrogenation of single-wall carbon nanotubes using high boiling point polyamines as hydrogenation reagents. The nature of the adsorption bond and preferential adsorption geometries were characterized for different hydrogenation levels. The barrier for sigmatropic rearrangement of chemisorbed hydrogen atoms was found to be about 1 $\mathrm{eV}$, thus facilitating surface diffusion and formation of energetically favored, axially aligned adsorbate chains. Chemisorbed hydrogen modified the structure and stability of nanotubes significantly and increased the inter-tube distance, thus explaining improved dispersability in solvents like methanol, ethanol, chloroform, and benzene.

The recently demonstrated hydrogenation of the $\mathrm{C}_{60}$ fullerene by diethylenetriamine reagent was studied using $a b$ initio density functional calculations. Results indicated that the optimal monoaddition reaction is exothermic, with approximately $0.5 \mathrm{eV}$ activation barrier associated with the simultaneous docking of the polyamine functional group and $\mathrm{H}$ transfer to $\mathrm{C}_{60}$. The calculated vibrational frequencies provide to experimentally confirmation of the presence of hydroamination, a necessary prerequisite for successful hydrogenation of $\mathrm{C}_{60}$ by this technique.

\section{Experimental Characterization of Storage Materials}

Complex metal hydrides $\left(\mathrm{NaBH}_{4}, \mathrm{NaAlH}_{4}, \mathrm{Li}_{3} \mathrm{AlH}_{6}, \mathrm{RbBH}_{4}\right.$ and $\left.\mathrm{KBH}_{4}\right)$ were studied using collaborative theory and experiment. High pressure X-ray diffraction and high temperature X-ray diffraction measurements of structural phase changes were made at the Advanced Photon Source, ANL. These observations allowed validation of theoretical calculations for alkali-borohydrides such as $\mathrm{NaBH}_{4}$ and $\mathrm{KBH}_{4}$. These undergo transitions from the cubic phase to the tetragonal phase, followed by another transition to the orthorhombic phase, while $\mathrm{NaAlH}_{4}$ undergoes a structural transition from tetragonal to monoclinic phases under pressure. High pressure phase transformations were observed by x-ray diffraction and Raman scattering measurements and confirmed by ab initio calculations using the local density approximation (LDA) and the generalized gradient approximation (GGA). Structural studies at high pressure are necessary and important to understand the synthesized new compounds and the use of ball-milling to enhance the dehydrogenation process.

Surfaces of carbon nanomaterials (single-walled carbon nanotubes - SWNT, $\mathrm{C}_{60}$, and graphite) were studied using a variety of laterally integrating and locally resolving 
experimental techniques, covering both spectroscopic and microscopic aspects. Our studies focused on the electronic surface structure, surface contamination, and, in the case of SWNTs, the identification of chirality and the associated metallic or semiconducting character. Utilizing a custom-built hydrogen source, which allows the exposure of carbon nanomaterials to atomic and molecular hydrogen beams, we studied the hydrogen sorption behavior of these carbon nanomaterials, in particular SWNTs (the results were presented at the Fall 2008 Materials Research Society meeting in Boston and were awarded one of the prestigious MRS poster awards).

In a detailed study of the interaction between metal adsorbates (in particular Ti) and carbon nanomaterials, we focused on an understanding of the hydrogenation processes and their competition with oxidation processes. In laterally integrating $\mathrm{x}$-ray photoelectron spectroscopy (XPS) studies, we could demonstrate a significant $\mathrm{Ti}$ oxidation behavior and demonstrate that such processes will make it impossible to use metallic Ti adatoms as a hydrogen storage enhancement, unless it is possible to reverse the Ti oxidation during (or immediately prior to) the loading cycle. We could show that Li co-adsorption, which is easily performed even on an industrial scale, offers such a solution to the Ti-oxidation issue (J. Am. Chem. Soc. 132, 5789-5792 (2010)).

Furthermore, we have established and employed the capabilities to prepare and image individual SWNTs with the purpose of determining their chirality and local electronic structure. Moreover, we have established the capabilities to monitor such individual SWNTs under in-situ metal decoration, e.g., with Ti and/or Li atoms, and the additional in-situ exposure to atomic and/or molecular hydrogen beams using the above-mentioned custom-built hydrogen source. First experiments confirm our capabilities to monitor individual SWNTs, to deposit small Ti clusters, and to determine the local electronic structure near the metal-SWNT interfaces. Also, initial hydrogenation steps could be performed during this project, and further work will be performed if suitable funding can be secured.

\section{Development of Conducting Polymeric Storage Material}

Polymer was synthesized chemically and electrochemically, incorporating $\operatorname{Pd}($ ii) and Pd(iv) species in both materials. A total of twelve different materials were produced. Contrary to many reports in the open literature, these materials do not require liquid nitrogen temperatures to observe hydrogen sorption. We have evaluated the reproducibility of the sorption/desorption of hydrogen for all of the materials produced and have identified the two most promising PANI/Pd composites. The unsorbed hydrogen elutes in less than one minute with the sorbed hydrogen elution initiated at $\sim 3.5$ minutes. The elution of sorbed hydrogen is complete within a minute. In addition, variations in exposure times did not result in large differences in the quantity of sorbed hydrogen, indicating fast adsorption kinetics. Observations showed that temperature influences the desorption of hydrogen from the composites materials. The percent of desorbed hydrogen measured at $200{ }^{\circ} \mathrm{C}$ reached a maximum value of $1.7 \%$ by weight. A value of $0.5 \%$ was obtained for the same composite material at a desorption temperature of $160^{\circ} \mathrm{C}$. 
For comparison, the hydrogen sorption by the Aniline/Pd(ii) composite was independently measured at the Southwest Research Institute (SWRI). The institute is designated as the independent measurement facility for hydrogen sorption by the Department of Energy.

The SWRI data showed maximum hydrogen sorption of $0.8 \%$ for the composite held at room temperature, as a function of equilibrium pressure. The desorption temperature was $175^{\circ} \mathrm{C}$. The temperature is intermediate between the $160^{\circ} \mathrm{C}$ and $200^{\circ} \mathrm{C}$ reported above, and consequently the SWRI-reported hydrogen desorption values were between those values reported above. The comparison verifies the sorption values obtained in our laboratory for the composite material. More importantly, the percent hydrogen sorption observed for the composite is high for materials at room temperature based on the literature to date.

The data obtained from these studies suggest a very interesting behavior of the material under cyclic testing. While the composite material provided for independent testing showed no hydrogen sorption on the first trial, each subsequent trial showed reproducible hydrogen sorption. This implies that the material undergoes a conditioning process during the first trial. In addition, the adsorption/desorption isotherms for the trials showing hydrogen sorption did not exhibit any appreciable hysteresis, indicating that the adsorption/desorption is reversible.

\section{Development of Durable and Inexpensive PEM for Fuel Cells}

A new type of sulfonated aromatic polyarylenes as candidate building blocks for proton exchange membranes was conceived. Density functional theory calculations and $a b$ initio molecular dynamics simulations suggested that desulfonation is limited at high temperatures, owing to the strong aryl- $\mathrm{SO}_{3} \mathrm{H}$ bond induced by the electron-deficient aromatic ring, and that the proposed polymers exhibit good thermomechanical stability due to the robust aromatic main-chain repeating unit. Simulations also emphasize the importance of the Grotthuss-type mechanism, with interconversion between Eigen $\left(\mathrm{H}_{9} \mathrm{O}_{4}{ }^{+}\right)$and $\mathrm{Zundel}$ cations $\left(\mathrm{H}_{5} \mathrm{O}_{2}{ }^{+}\right)$as limiting structures for the hydrated proton transport in the vicinity of the sulfonic acid groups.

Aromatic polyamides are known to have excellent chemical stability and mechanical properties. Initially prepared sulfonated aromatic poly(ether amide)s showed promising proton conductivity, comparable to that of Nafion at $80{ }^{\circ} \mathrm{C}$ and $100 \%$ relative humidity, but poor stability was found when ion exchange capacity (IEC) was increased to improve the proton conductivity further. To achieve this goal without excessive water uptake, synthesis techniques were developed to incorporate fluorinated moiety along the polymer chains. Polycondensation reactions of various diamines, terephthalic acid (TA), and sulfonated terephthalic acid (STA) produced high-molecular-weight sulfonated aromatic poly(ether amide)s. By changing the ratio of TA and STA, sulfonated polymers with different degrees of sulfonation could be obtained. Based on intrinsic viscosity measurement results, all sulfonated polymers were found to have high molecular weights (intrinsic viscosity $>1.0 \mathrm{dL} / \mathrm{g}$ ). Synthesized membrane properties demonstrated ion exchange capacities (IEC) in the range of other hydrocarbon-based proton exchange 
membranes, along with relatively lower water uptake values compared to membranes with similar IEC. High molar STA membranes were synthesized to improve proton conductivity but these highly sulfonated polymer samples became unstable in water. In response, fluorine substituted sulfonated poly(ether amide)s were prepared. Fluorine substitution in the sulfonated polymers resulted in a significant reduction of water uptake compared to that of non-fluorinated sulfonated polymers, allowing higher concentration of sulfonic acid moiety in the polymer. The fluorine-substituted series ionomers showed higher conductivity than the non-fluorinated polymer and Nafion when measured at $100 \%$ relative humidity and varying temperatures.

\section{Theoretical and Experimental Studies of PEM Catalysts}

In our theory efforts, the electronic structures and stability of small Ti clusters was studied to establish their reactivity. The sequential growth of titanium clusters up to 15 atoms, and for the first time, the dissociative chemisorption of $\mathrm{H}_{2}$ on the corresponding minimum energy clusters were investigated using DFT. The studies showed a strong correlation between the chemisorption energy and cluster size and were published in $J$. Phys. Chem. C (2007).

Theoretical studies of chemisorption have generally been limited to a single adsorbate molecule interacting with a metal surface or cluster. The effect of hydrogen saturation on the electronic structures of small Ti clusters was studied for more realistic models of titanium catalytic and hydrogen storage systems. Calculations on $\mathrm{Ti}_{4}, \mathrm{Ti}_{7}, \mathrm{Ti}_{13}$, and the larger $\mathrm{Ti}_{55}$ cluster revealed that at high hydrogen concentrations, hydrogen multicenter bonds become prevalent. A key result is that the hydrogen multicenter bonds are characterized by vibrational frequencies in the $1100-1500 \mathrm{~cm}^{-1}$ range, and that adsorbed hydrogen could be easily released by selective excitation of vibrational modes. Due to its high stability, most of the studies focused on different conformers of $\mathrm{Ti}_{13}$, but hydrogen saturation $\mathrm{Sc}_{13}$ and $\mathrm{Zr}_{13}$ clusters was also investigated. These studies led to a series of papers: J. Phys. Chem. A (2008), J. Chem. Phys. (2008, 2009), and Phys. Rev. B (2009).

In a parallel work, small clusters of Pd and Pt as well as Pt-Co alloy systems were studied in collaboration with Dr. Hansong Cheng to explore how alloying and doping affect reactivity of small transition metal clusters. The growth of small Pt clusters on $\alpha-\mathrm{Al}_{2} \mathrm{O}_{3}$ surfaces was applied to investigate the nature of cluster-substrate interaction. Though electronic structures of subnanoclusters of $\mathrm{Pt}$ and $\mathrm{Pd}$ have been extensively investigated, their interaction with support materials has not been well understood. The works on $\mathrm{Pd}$ clusters and growth of Pt clusters on $\mathrm{Al}_{2} \mathrm{O}_{3}$ support were published in 2007. The work on $\mathrm{H}_{2}, \mathrm{O}_{2}$, and $\mathrm{CO}$ chemisorption on $\mathrm{Pt}_{4}$ and $\mathrm{Pt}_{3} \mathrm{Co}$ clusters was published in J. Chem. Phys. in 2008.

In our experimental studies of PEM fuel cell catalysts, we teamed up with UTC Power, Inc., to conduct a detailed analysis of the electronic and chemical surface properties of nanomaterial fuel cell catalysts from UTC. For this purpose, we investigated a large variety of Pt-Co, Pt-Ir, and Pt-Ir-Co nanocatalysts and reference materials for fuel cell applications with photoelectron spectroscopy (at UNLV) and x-ray absorption and x-ray emission spectroscopy (at the Advanced Light Source in Berkeley). Primary emphasis 
was placed on an analysis of the emergence of potential core-shell structures as a function of sample treatments (e.g., annealing procedures and acid leach processes). Detailed insights into the chemical surface structure and the electronic properties could be gained and directly fed back to the preparation processes at UTC.

In detail, our experiments could identify experimental annealing conditions that lead to the formation of electronic structures that are different from mere superpositions of the electronic structures of the constituent metals. Some evidence could be found for a Pt-Co core shell structure formation, but in-situ performed acid leach tests could not confirm that this core-shell structure can be achieved reproducibly. Annealing studies indicated optimal preparation conditions for custom-tailored valence structures that can aid in the catalytic activity of the nanoparticles. They furthermore shed light on the oxidation and hydroxide-formation at nanoparticle surfaces and on the complex compositional phase diagram of such ternary (Pt-Co-Ir) nanoparticles as a function of annealing temperature. 


\section{PUBLICATIONS}

Kumar, R.S. and A.L. Cornelius, "Structural Transitions in $\mathrm{NaBH}_{4}$ Under Pressure." Appl. Phys. Lett. 87, 261916 (2005).

Lee, T.G., N. Balakrishnan, R.C. Forrey, P.C. Stancil, D R. Schultz, and G.J. Ferland, "State-to-State Rotational Transitions in $\mathrm{H}_{2}+\mathrm{H}_{2}$ Collisions at Low Temperatures, J. Chem. Phys. 125, 114302 (2006).

Ma H. and T. Pang, "Path-integral Quantum Monte Carlo Study of a Mixture of BoseEinstein Condensates," Physics Letters A 351, $92-96$ (2006).

Pang, T. "An Introduction to Computational Physics, $2^{\text {nd }}$ Edition" (Cambridge University Press, Cambridge, UK, 2006), 402 pp.

Weck, P.F., N. Balakrishnan, J. Brandao, C. Rosa, and W. Wang, "Dynamics of the $\mathrm{O}\left({ }^{3} \mathrm{P}\right)+\mathrm{H}_{2}$ reaction at low temperatures: Comparison of quasiclassical trajectory with quantum scattering calculations," J. Chem. Phys. 124, 074308 (2006).

Dhilip Kumar, T. J., P. F. Weck, and N. Balakrishnan, "Evolution of small Ti clusters and the dissociative chemisorption of $\mathrm{H}_{2}$ on Ti”, J. Phys. Chem. C 111, 7494-7500 (2007).

Kim, E., R. Kumar, P.F. Weck, A.L. Cornelius, M. Nicol, S.C. Vogel, J. Zhang, M. Hartl, L. Daemen, and Y. Zhao, "Pressure-driven phase transitions in $\mathrm{NaBH}_{4}$ : Theory and experiments." J. Phys. Chem. B 111, 11873 (2007).

Kim, E., T. Pang, W. Utsumi, V. L. Solozhenko, and Y. Zhao, " Cubic phases of $\mathrm{BC}_{2} \mathrm{~N}$ : A first-principles study ", Phys. Rev. B. 75, 184115 (2007).

Kumar, R. S., A.L. Cornelius, M.G. Pravica, M.F. Nicol, M.Y. Hu, and P.C. Chow, "Bonding changes in single wall carbon nanotubes (SWCNT) on $\mathrm{Ti}$ and $\mathrm{TiH}_{2}$ addition probed by X-ray Raman scattering." Diamond and Related Mater. 16, 1136 (2007).

Kumar, Ravhi S., Eunja Kim, Oliver Tschauner, Andrew L. Cornelius, Martin P. Sulic, and Craig M. Jensen, "Pressure-induced structural phase transition in $\mathrm{NaAlH}_{4}$." Phys. Rev. B 75, 174110 (2007).

Weck, P. F., T. J. Dhilip, R. Kumar, E. Kim, and N. Balakrishnan, " Computational study of hydrogen storage in organometallic compounds ", J. Chem. Phys., 126, 094703 (2007).

Weck, P. F., E. Kim, N. Balakrishnan, H. Cheng, B. Yakobson " Designing carbon nanoframeworks tailored for hydrogen storage ", Chem. Phys. Lett. 439, 354 (2007).

Weck, P. F., E. Kim, N. Balakrishnan, F. Poineau, C. B. Yeamans, K. R. Czerwinski, " First-principles study of single-crystal uranium mono- and dinitride ", Chem. Phys. Lett., 443, 82 (2007). 
Zhou, C., J. Wu, T. J. Dhilip Kumar, N. Balakrishnan, R. C. Forrey, and H. Cheng, “The Growth Pathway of Pt Clusters on $\alpha-\mathrm{Al}_{2} \mathrm{O}_{3}$ (0001) Surface", J. Phys. Chem. C 111, 13786-13793 (2007).

Zhou, C., C. Luo, J. Wu, T. J. Dhilip Kumar, N. Balakrishnan, R. C. Forrey, and H. Hansong, "First principles study of small palladium cluster growth and isomerization", Int. J. Quant. Chem. 107, 1632-1641 (2007).

Dhilip Kumar, T. J., P. Tarakeshwar, and N. Balakrishnan, "Structural, energetic and electronic properties of hydrogenated titanium clusters", J. Chem. Phys. 128, 194714 (2008).

Dhilip Kumar, T. J., C. Zhou, H. Cheng, R. C. Forrey, and N. Balakrishnan, "Effect of Co doping on the catalytic activity of small Pt clusters", J. Chem. Phys. 128, 124704 (2008).

Miller, G., J. Kintigh, E. Kim, P. F. Weck, S. Berber, and D. Tomanek, " Hydrogenation of Single-Wall Carbon Nanotubes Using Polyamine Reagents: Combined Experimental and Theoretical Study" ", J. Am. Chem. Soc. 130, 2296 (2008).

Kalita, P.E., A.L. Cornelius, K.E. Lipinska-Kalita, Cedric L. Gobin, H. Peter Liermann, "In situ observations of temperature- and pressure-induced phase transitions in $\mathrm{TiH}_{2}$ : Angle-dispersive and synchrotron energy-dispersive X-ray diffraction studies." J. Phys. Chem. Solid 69, 2240 (2008).

Kim, E., P. Weck, B. Naduvalath, and C. Bae, "Nanoscale Building Blocks for the Development of Novel Proton-exchange Membrane Fuel Cells" J. Phys. Chem. B (Letter), 112, 3283 (2008). [March 20, 2008 Cover Page].

Kim, E., P. Weck, S. Berber, and D. Tomanek, "Mechanism of fullerene hydrogenation by polyamines: $a b$ initio density functional calculations", Phys. Rev. B 78, 113404 (2008).

Kumar, R., E. Kim, and A.L. Cornelius, "Structural Phase Transitions in the Potential Hydrogen Storage Compound $\mathrm{KBH}_{4}$ under Compression." J. Phys. Chem. C 112 (2008) 8452.

Kumar, Ravhi S., Xuezhi Ke, Andrew L. Cornelius, and Changfeng Chen, "Effect of Pressure and Temperature on Structural Stability of Potential Hydrogen Storage Compound $\mathrm{Li}_{3} \mathrm{AlH}_{6} . "$ Chem. Phys. Lett. 460, 442 (2008).

Kumar, Ravhi S., and Andrew L. Cornelius, "Structural phase transitions in the potential hydrogen storage compound $\mathrm{RbBH}_{4}$ under compression." J.Alloys and Comp. 476, 5 (2008). 
Tarakeshwar, P., T. J. Dhilip Kumar, and N. Balakrishnan, "Nature of hydrogen interaction and saturation on small Titanium clusters", J. Phys. Chem. C 112, 2846-2854 (2008).

Tkachev, S., M. Pravica, S. E. Romano, and P. Weck, "High pressure studies of 1,3,5,7cyclooctatetraene: Experiment and Theory", J. Phys. Chem. A., 112, 11501 (2008).

Weck, P., E. Kim, S. Lepp, B. Naduvalath, and H. Sadeghpour, "Dimer-Induced Stabilization of H Adsorbate Cluster on BN(0001) Surface", Phys. Chem. Chem. Phys. 10 (Communication), 5184 (2008). [September 14, 2008 Cover Page].

Dhilip Kumar, T. J., P. Tarakeshwar, and N. Balakrishnan, "Geometric and Electronic Structures of Hydrogenated Transition Metal ( $\mathrm{M}=\mathrm{Sc}, \mathrm{Ti}, \mathrm{Zr})$ clusters", Phys. Rev. B 79, 205415 (2009).

Jo, T. S., C. H. Ozawa, B. R. Eagar, L. V. Brownell, D. Han, C. Bae, "Synthesis of Sulfonated Aromatic Poly(ether amide)s and Their Application to Proton Exchange Membrane Fuel Cells", J. Polym. Sci., Part A: Polym. Chem. 2009, 47, 485-496.

Lee, S., E. Kim and C. Kim, "Unzipping of single-walled carbon nanotubes: A tightbinding molecular-dynamics study", J. Kor. Phys. Soc., Vol. 54, 682 (2009).

Tarakeshwar, P., T. J. Dhilip Kumar, and N. Balakrishnan, "Hydrogen multicenter bonds and reversible hydrogen storage", J. Chem. Phys. 130, 114301 (2009).

Tran I., R. Felix, M. Bär, L. Weinhardt, Y. Zhang, and Heske, C., "Oxidation of Titanium-decorated Single-Walled Carbon Nanotubes and subsequent reduction by Lithium", J. Am. Chem. Soc. 132, 5789-5792 (2010). 


\section{MANUSCRIPTS IN PREPARATION}

Zhang, Y. et al., "Electronic structure of Pt-based nanocatalysts", in preparation for J. Phys. Chem.

Tran, I. et al., "Oxidation behavior of metal adatoms on carbon nanomaterials", in preparation for J. Phys. Chem.

Tran, I. et al., "Local atomic and electronic surface structure of carbon nanomaterials, metal adatoms, and adsorbed molecular species", in preparation for Surface Science.

Felix, R. et al., "Temperature Dependence of Tungsten-mediated Hydrogen Dissociation monitored by Hydrogen Chemisorption on Single-Walled Carbon Nanotubes", in preparation for J. Vacuum Science and Technology.

Mai, H., P. Tarakeshwar and N. Balakrishnan, "Charge and Adsorbate Saturation Effects on the Energies, Geometries, and Transition States of Doped Platinum Clusters".

Tarakeshwar, P. and N. Balakrishnan, "Low-cost alloy clusters for nanocatalysis". 


\section{PRESENTATIONS}

Giefers H., C. Gobin, B. Keifer, E. Kim, Y. Lee, M. Nicol, J. Orwig, M. Pravica, O. Tschauner, B. Yulga, 'X-ray diddraction and spectroscopy at high pressures: Recent studies', SRMS-5 Conference, Chicago, July 30 - August 2, 2006.

Kim E., "Instability of carbon nanotubes: Molecular-dynamics Simulations", International Symposium on Materials Issues in Hydrogen Production and Storage, Santa Barbara, CA, August 20-25, 2006.

Weck P.F., T.J. Dhilip Kumar, E. Kim, and N. Balakrishnan, "Computational study of hydrogen storage in organometallic compounds", International Symposium on Materials Issues in Hydrogen Production and Storage, Santa Barbara, CA, August 20-25, 2006.

Kim E., "Theoretical study of solid sodium borohydrides under Pressure", MRS Fall meeting, Boston, MA, November 26 - 28, 2006.

Kim E., D. Stucke, and T. Pang, "Oxidation of Pt (100) surface: Ab initio studies", 2007 APS March Meeting, Denver, CO, March 5-9, 2007.

T. J. Dhilip Kumar, P. F. Weck, and Balakrishnan Naduvalath, Evolution of small Ti clusters and the dissociative chemisorption of $\mathrm{H}_{2}$ on Ti, APS March Meeting, March 5-9, 2007, Denver, CO.

T. J. Dhilip Kumar, C. Zhou, and Balakrishnan Naduvalath, First principles study of adsorption and dissociation of $\mathrm{H}_{2}, \mathrm{O}_{2}$, and $\mathrm{CO}$ on $\alpha-\mathrm{Al}_{2} \mathrm{O}_{3}$ (0001) supported Pt-Co alloy, APS March Meeting, March 5-9, 2007, Denver, CO.

P. F. Weck, E. Kim, N. Balakrishnan , H. Cheng, "Design of tailored carbon nanostructures for hydrogen storage", 2007 APS March Meeting, Denver, CO, March 59, 2007.

Balakrishnan Naduvalath, Molecular adsorption and dissociation on transition metal clusters and alloys, presentation to representatives from United Technology Corporation, March 28, 2007, UNLV.

Kim E., R. Kumar, A. Cornelius, N. Micol, S. C. Vogel, J. Zhang, M. Hartl, A. C. Stowe, L. Daemen, and Y. Zhao, "Pressure-induced structural transitions in $\mathrm{NaBH}_{4}$ ", SMEC 2007 meeting, Miami, FL, April 15-20, 2007.

T. J. Dhilip Kumar, C. Zhou, and Balakrishnan Naduvalath, R. C. Forrey and H. Cheng, First principles study of adsorption and dissociation of $\mathrm{H}_{2}, \mathrm{O}_{2}$, and $\mathrm{CO}$ on $\mathrm{Pt}_{4}$ and $\mathrm{Pt}_{3} \mathrm{Co}$ clusters, DAMOP Meeting, June 5-9, 2007, Calgary, Canada.

Kim E., R. Kumar, A. Cornelius, M. Nicol, S. Vogel, J. Zhang, M. Hartl, A. Stowe, L. Daemen, and Y. Zhao, "Structural characterization of sodium borohydrides: Theory and 
Experiments", International Symposium on Materials Issues in a Hydrogen Economy, November 12-15, 2007, Richmond, VA.

R. Kumar, E. Kim, and A. Cornelius, "Structural changes in RbBH4 under pressure investigated by Synchrotron powder X-ray diffraction and theoretical studies", International Symposium on Materials Issues in a Hydrogen Economy, November 12-15, 2007, Richmond, VA.

P. Weck, E. Kim, N. Balakrishnan, H. Cheng, and B. Yakobson, "Lithium-decorated carbon nanoframeworks tailored for hydrogen storage", International Symposium on Materials Issues in a Hydrogen Economy, November 12-15, 2007, Richmond, VA.

T. J. Dhilip Kumar, P. Tarakeshwar, and N. Balakrishnan, Sequential Dissociative Chemisorption of $\mathrm{H}_{2}$ on $\mathrm{Ti}_{13}$ Cluster, APS March Meeting, March 10-14, 2008, New Orleans, Louisiana.

P. Tarakeshwar, T. J. Dhilip Kumar, and N. Balakrishnan, Hydrogen Multicenter Bonds on Small Metal Clusters, APS March Meeting, March 10-14, 2008, New Orleans, Louisiana.

P. F. Weck, E. Kim, C. Bae, and N. Balakrishnan, Nanoscale building blocks for the development of novel proton-exchange membranes fuel cells: A first-principles study, APS March Meeting, March 10-14, 2008, New Orleans, Louisiana.

Kim E., and T. Pang, "First-principles study of the oxygen-reduction reaction on the Pt(100) surface," American Physical Society Annual Meeting, New Orleans, Louisiana, March 10-14, 2008.

C. Bae, "New Functional Polymers for Alternative Energy Application" UNLV Renewable Energy Symposium, Las Vegas, NV, August 20, 2008.

Tarakeshwar Pilarisetty, Dhilip Kumar, Thogluva Janardhanan, and N. Balakrishnan, Hydrogen storage and catalytic properties of transition metal clusters: Bonding changes and saturation effects, 236th American Chemical Society National Meeting, Philadelphia, PA, August 17-21, 2008.

T. S. Jo, C. Bae "Synthesis of Ionic Conducting Polymers for Fuel Cell Applications and Biodiesel Productions" 42 ${ }^{\text {nd }}$ Western Regional Meeting of the American Chemical Society, Las Vegas, NV, September 23-27, 2008.

T. J. Dhilip Kumar, P. Tarakeshwar, and N. Balakrishnan, Geometric and Electronic Structures of Ti-Al and Hydrogenated Ti-Al Nanoclusters, American Chemical Society Western Regional Meeting, Las Vegas, NV, September 24-28, 2008. 
T. J. Dhilip Kumar, P. Tarakeshwar, and N. Balakrishnan, The Effect of Alloying on CO Tolerance of Pt Nanoparticles, American Chemical Society Western Regional Meeting, Las Vegas, NV, September 24-28, 2008.

Heske, C., "Hydrogen Fuel Cells and Storage Technology", presentation to the FreedomCAR Initiative, November 10, 2005.

Heske, C., "X-ray Spectroscopy of Buried Things: Interfaces, Liquids and Dirty Powders", Stanford Synchrotron Radiation Laboratory, February 27, 2006.

Heske, C., "Hydrogen Fuel Cells and Storage Technology", Ford Motor Company, March 29, 2006.

Heske, C., "Hydrogen Fuel Cells and Storage Technology Project at UNLV", DOE Hydrogen Review Meeting, Arlington, VA, May 17, 2006.

Heske, C., "Surface and Interface Analysis at UNLV", United Technologies Company Fuel Cells, Hartford, CT, June 23, 2006.

Heske, C., "UNLV Research Foundation - overview of hydrogen research projects", United Technologies Research Center, Hartford, CT, June 23, 2006.

Heske, C., "Hydrogen Fuel Cells and Storage Technology Project at UNLV", United Technologies Research Center, Hartford, CT, June 23, 2006.

Heske, C., "Surface and interface analysis in Las Vegas", Seminar Experimentelle Physik II, University of Würzburg, Germany, July 20, 2006.

Heske, C., "How to reveal the chemical and electronic properties of interfaces, buried layers, and liquids with soft x-ray spectroscopy", Materials Science and Engineering Department, Stanford University, August 31, 2006.

Weinhardt, L., FCAST Project Meeting, November 16, 2006.

Heske, C., "The surface and interface analysis "tool chest" at UNLV", Group seminar Prof. T. Moustakas, Boston University, November 17, 2006.

Heske, C., "How to reveal the chemical and electronic properties of interfaces, buried layers, and liquids with soft x-ray spectroscopy", Physics Department, Boston University, December 8, 2006.

Heske, C., "Hydrogen Fuel Cells and Storage Technology", FCAST Project Presentation (Storage only) to the Technical Team Meeting, FreedomCAR, Detroit, December 19, 2006. 
C. Heske and B. Naduvalath, DOE Hydrogen Review Meeting, Arlington, VA, May 2007.

Hemmers, O., K. Lipinska-Kalita, D. Lindle, I. Demchenko and W.C. Stolte, Poster presented at UNLV Renewable Energy Symposium, Las Vegas August 2007: "Synchrotron X-Ray Spectroscopy Studies for H2 Storage."

Heske, C., "Understanding and Optimizing Surfaces and Interfaces in Energy Conversion Devices", 2007 Annual Energy Symposium, UNLV, August 16, 2007 (invited oral).

Hemmers, O., K. Lipinska-Kalita, D.W. Lindle I. Demchenko, and W.C. Stolte Poster presented at Users' Association Annual Meeting, Berkeley, CA, October 4-6, 2007, "Synchrotron X-ray spectroscopy studies for H2 storage."

Demchenko, I.N., W.C. Stolte, N. Birknern, R. Minikayev, A. Wongjamras, and M. Chernyshova, Poster presented at ALS Users' Association Annual Meeting, Berkeley, CA, October 4-6, 2007, "Transmission versus fluorescence mode collection in XAS: Ti K-edge of TiN."

Tran, I.C., R. Felix, L. Weinhardt, M. Bär, and Heske, C., "Ti-coated Single-walled Carbon Nanotubes for Hydrogen Storage: A Spectroscopic and Microscopic Study", 2007 Fall MRS Meeting, Boston, MA, November 2007 (poster).

Heske, C., "Soft x-rays and the electronic structure of buried things - interfaces, liquids, and below dirty surfaces", Materials Science Institute, University of Oregon, Eugene, February 26, 2008.

Heske, C., Presentation during the site visit of Jesse Adams, DOE Golden Field Office, on March 14, 2008.

Heske C., and N. Balakrishnan, "Hydrogen Fuel Cells and Storage Technology Project", Presentation to the DOE Hydrogen Storage Tech Team, FreedomCAR, Detroit, April 17, 2008.

Heske C., and N. Balakrishnan, "Hydrogen Fuel Cells and Storage Technology Project", Poster presentation at the DOE Program Review Meeting, Arlington, June 9, 2008.

Zhang, Y., L. Weinhardt, T. Hofmann, M. Bär, Heske, C., and T.T. Aindow, "The chemical and electronic structure of platinum-based nanoparticle catalysts for PEM fuel cells", American Chemical Society Western Regional Meeting, Las Vegas, September 24, 2008 (invited oral).

Felix, R., Ich Tran, Lothar Weinhardt, Marcus Bär, Timo Hofmann, Yufeng Zhang, and Clemens Heske, "Study of the Interaction between Hydrogen and Carbon-based 
Nanomaterials", 2008 MRS Fall Meeting, Boston, MA, November 2008 (poster, won best poster award).

Tran I., "Microscopic and Spectroscopic Studies of Metal Deposition on Carbon-based Materials", Ph.D. thesis defense presentation, Department of Chemistry, UNLV, November 21, 2008.

Tran I., R. Felix, Y. Zhang, M. Bär, Heske, C., and L. Weinhardt, "Microscopic and Spectroscopic Studies of Metal Deposition on Carbon Nanomaterials", New Diamond and Nano Carbons Conference (NDNC 2009), June 7-11, 2009 Traverse City, MI (poster).

Heske, C., "Using soft x-rays to look at surfaces and interfaces for energy conversion and storage", National Renewable Energy Lab (NREL), June 21, 2010.

Heske, C., "Wie man mit Röntgenspektroskopie energierelevante Materialien und Systeme verstehen und verbessern kann“, Organisch-Chemisches Kolloquium im Sommersemester 2010, Karlsruhe Institute of Technology, July 2, 2010.

Heske, C., "Using soft x-rays to understand and improve materials for energy conversion devices", Department of Chemistry, University of California Santa Cruz, September 29, 2010 .

Heske, C., "Using soft x-rays to optimize materials for energy conversion devices", Department of Physics, Renmin University, Beijing, China, October 10, 2010.

Heske, C., "Using soft x-rays to understand and optimize materials for energy conversion", Department of Physics, Hanoi University of Science and Technology, Hanoi, Vietnam, November 1, 2010. 
ATTACHMENT A

Final Report, Clemens Heske, Department of Chemistry, University of Nevada, Las Vegas

This report covers the activities of the Heske Research Group as part of the UNLV RF FCAST (Fuel Cell And Storage Technology) project.

During the initial project year, these activities were part of Task 2 (Experiments, until 6/06). In the second project period, our activities contributed to Tasks 1 and 5 (7/06 - 6/09). In the final phase (7/09 - 12/10), Task 1 was continued as Task 2.2, and Task 2 was continued as Task 2.1.

\section{Project Title: Local spectroscopy of nanomaterials for hydrogen storage}

Project Period: 09/15/2005 - 12/31/2010

Date of Report: March 14, 2011

Contact: Clemens Heske, Department of Chemistry, UNLV

Project Objective: To study the electronic structure of nanomaterials for hydrogen storage in order to understand the detailed mechanisms of hydrogen adsorption and desorption (Task 1/Task 2.2). To collaborate with UTC to aid in the development of efficient catalysts for fuel cell applications (Task 5/Task 2.1).

\section{Executive Summary:}

Using and developing a tool chest of microscopy and spectroscopy techniques, this project has shed light on the chemical and electronic surface properties of candidate materials for hydrogen storage and fuel cell catalyst applications. In the case of hydrogen storage, detailed interactions between carbon nanomaterials, metal adatoms, and gaseous species (including atomic and molecular hydrogen) could be identified and mitigating procedures and parameters could be proposed. The studies on fuel cell catalyst nanoparticles, conducted in close collaboration with our project partner UTC, illuminated some of the complicated surface segregation phenomena observed after annealing and acid treatment steps. The results, once fully published, will contribute significant insights into the complicated aspects of nanomaterial surfaces for hydrogen storage and fuel cell applications which will need to be understood and controlled for insightbased optimization approaches. 
Results of Task 1/Task 2.2: Atomic and electronic structure of carbon-based nanomaterials for hydrogen-storage application

\section{Scanning probe microscopy}

To study the local atomic and electronic structure of carbon-based nanomaterials and their suitability as candidate materials for hydrogen storage applications, we began our project with the specification, procurement, and installation of a scanning probe microscope (Omicron, VTAFM XA, as seen in Fig. 1, bottom left corner). Significant efforts were devoted to commissioning of this instrument, including trouble-shooting of defective electronic components.

As shown in Fig. 2, atomic resolution was achieved in AFM and STM images of an HOPG surface at room temperature. Atomically resolved STM images were also achieved at low temperatures ( $40 \mathrm{~K}$ ), thus completing the demonstration of all capabilities of the instrument. For the experimental efforts in Task 1, we focused on finding and developing techniques to prepare suitable nanomaterial (nanotubes, C60, nanofibers) samples for the different experimental techniques (e.g., single separated nanotubes on a flat surface for SPM and thin homogeneous films for the laterally integrating spectroscopies). Furthermore, we collected first Raman and synchrotron-based photoemission data on commercial nanotube material, giving information about size and quality of the nanotube samples.

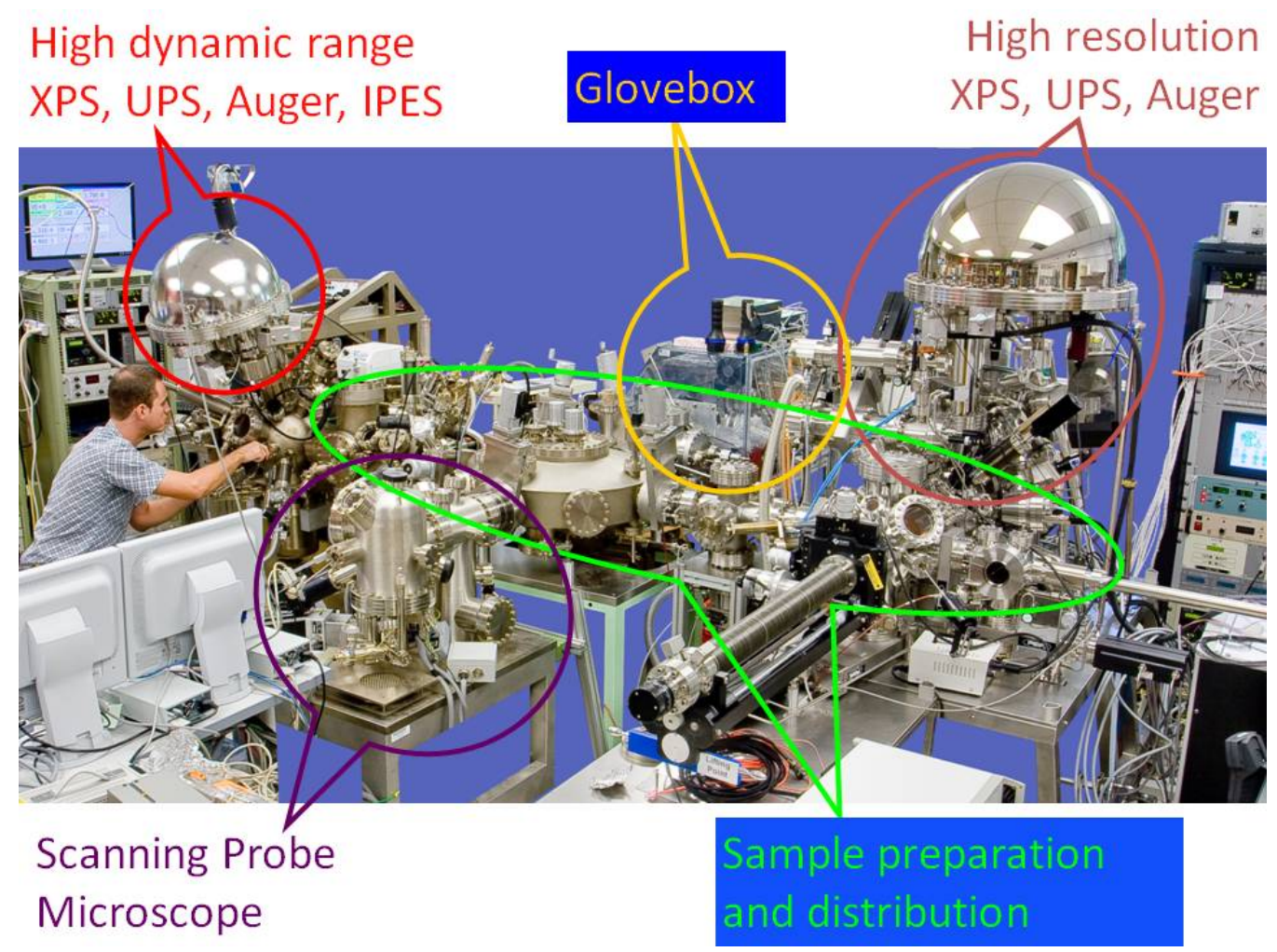

Fig. 1: Surface and Interface-Spectroscopy system at UNLV. Bottom left: Scanning Probe Microscope. 


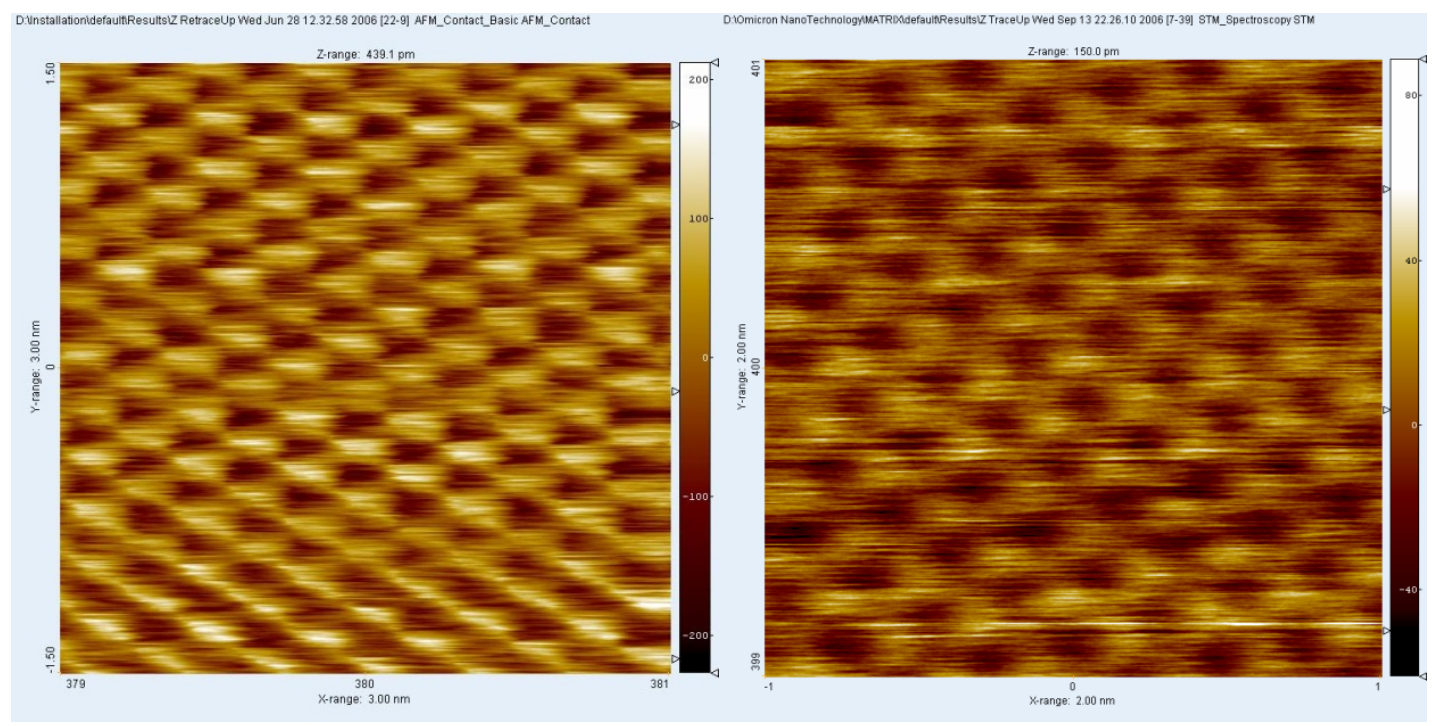

Fig. 2: AFM contact image (left) and STM image (right) of an HOPG surface, showing atomic resolution. The size of the AFM image is $3 \times 3 \mathrm{~nm}^{2}$, the size of the STM image is $2 \times 2 \mathrm{~nm}^{2}$.

To develop suitable techniques to prepare nanotube samples for characterization in the scanning probe microscope, we next focused on electrodepositing nanotubes on a highly-ordered pyrolytic graphite (HOPG) surface. For this approach a simple experimental set-up was constructed, as shown in Fig. 3. Nanotubes dispersed in water are brought between the HOPG substrate and a stainless steel plate. The nanotubes are then deposited by applying a voltage between the HOPG substrate and the steel plate.
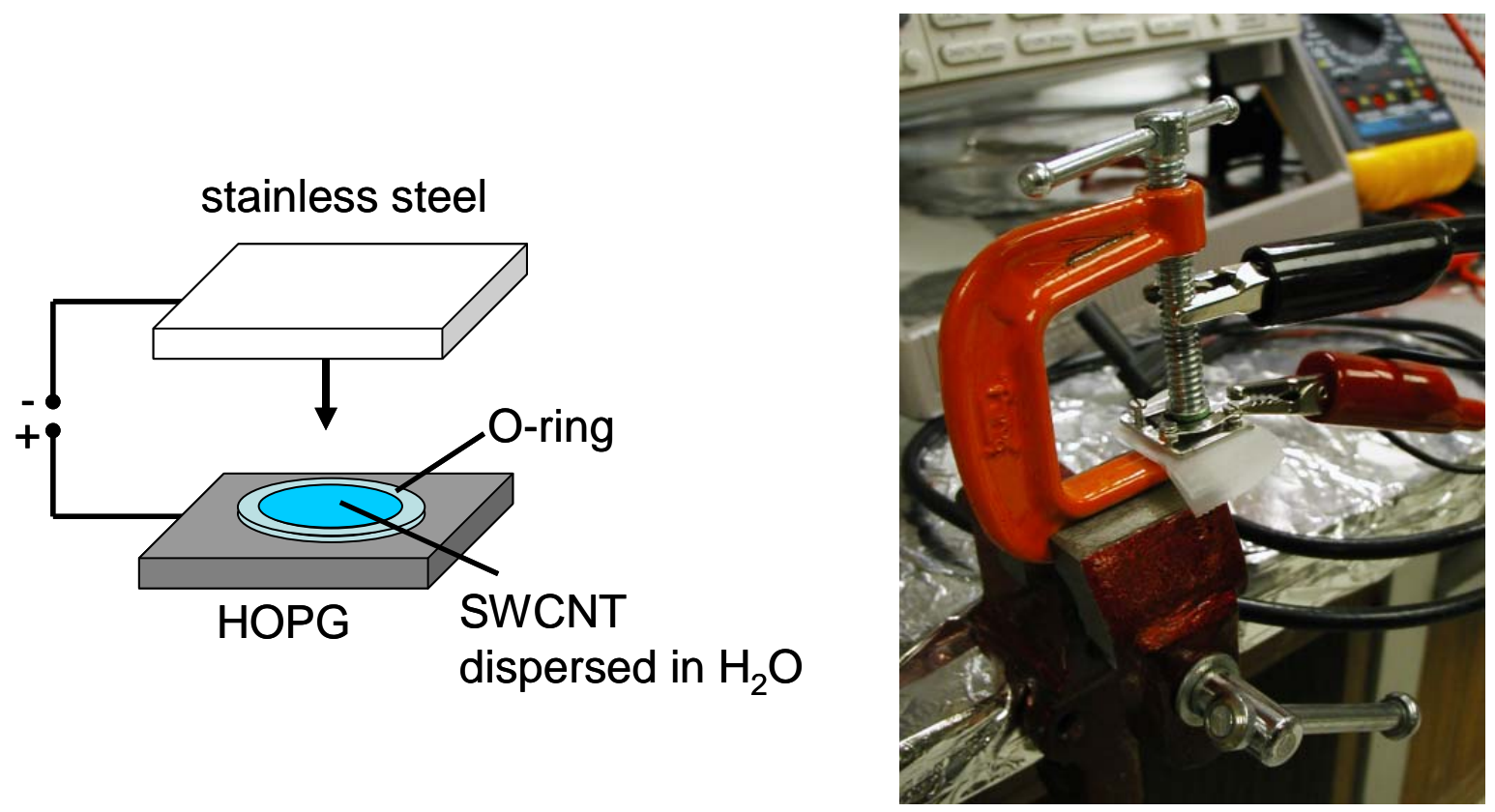

Fig. 3: Set-up for electro-deposition of carbon nanotubes. 

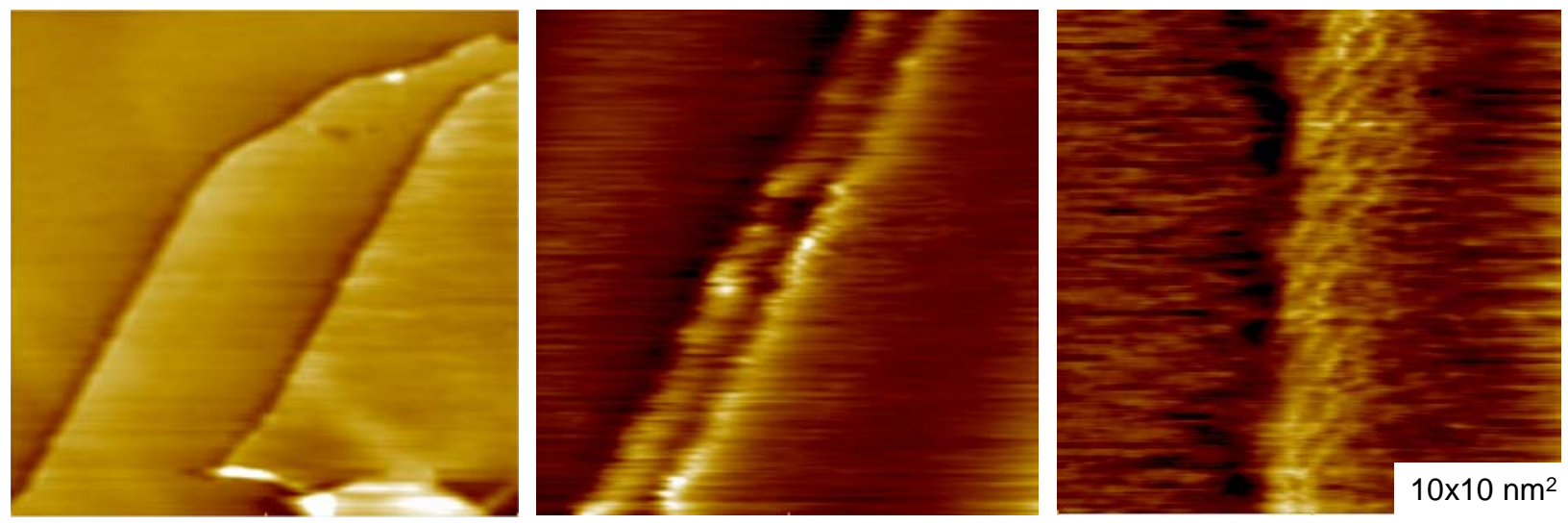

Fig. 4: Scanning Tunneling Microscopy pictures of SWCNT electrodeposited on an HOPG surface.

We investigated different samples prepared by electro-deposition with our scanning probe microscope. When depositing with the substrate on the bottom of the set-up (as shown in Fig. 3) we find a lot of bundled SWCNT on the graphite surface. This is presumably due to sedimentation of nanotubes caused by gravity during the deposition process. To avoid this effect, we have prepared another sample with an inverted set-up, i.e., with the HOPG on top and the stainless steel plate on the bottom. We find that this approach yields a low coverage of SWCNT on the HOPG surface as shown in Fig. 4, which is ideal to investigate isolated SWCNT. When "zooming in" we find that (in some cases) small bundles (e.g. two-bundled tubes, see center picture of Fig. 4) are formed. We were able to achieve atomic resolution on an isolated SWCNT as shown in the right picture in Fig. 4.

To understand the growth mode of a Ti-submonolayer we have deposited Ti on a Au/Si substrate (which serves as the substrate for our scanning probe experiments on individual SWCNTs). As an example, Fig. 5 shows an STM image of Ti nanoclusters that form after Ti deposition onto the $\mathrm{Au} / \mathrm{Si}$ substrate. Fig. 6 shows a similar experiment (i.e., Ti submonolayer deposition) on a Au/Si substrate with individual SWCNTs. While individual SWCNTs can be imaged with atomic resolution (see Fig. 4), the adsorption of Ti leads to a "blurring" of the lateral corrugation of the surface electronic structure and hence no atomically resolved images of the SWCNT could be obtained in this experimental run.

We have furthermore studied the morphology and electronic structure of a Au surface after Li deposition ( $\mathrm{Li} / \mathrm{Au}$ ). This system is compared to the Au surface after Ti deposition (Ti/Au) and used as a reference for investigating the $\mathrm{Li}$ behavior on carbon-based nanomaterials and its impact on Hydrogen storage applications. A layer of Au was deposited on a Si wafer and exposed to a Li dispenser element getter source for 2 minutes, resulting in a $2.8 \mathrm{~nm}$ thick Li film. Fig. 7 shows a scanning tunneling microscopy (STM) image of the Li/Au sample, and further aspects of this deposition series will be discussed further below (in conjunction with the corresponding XPS data). 


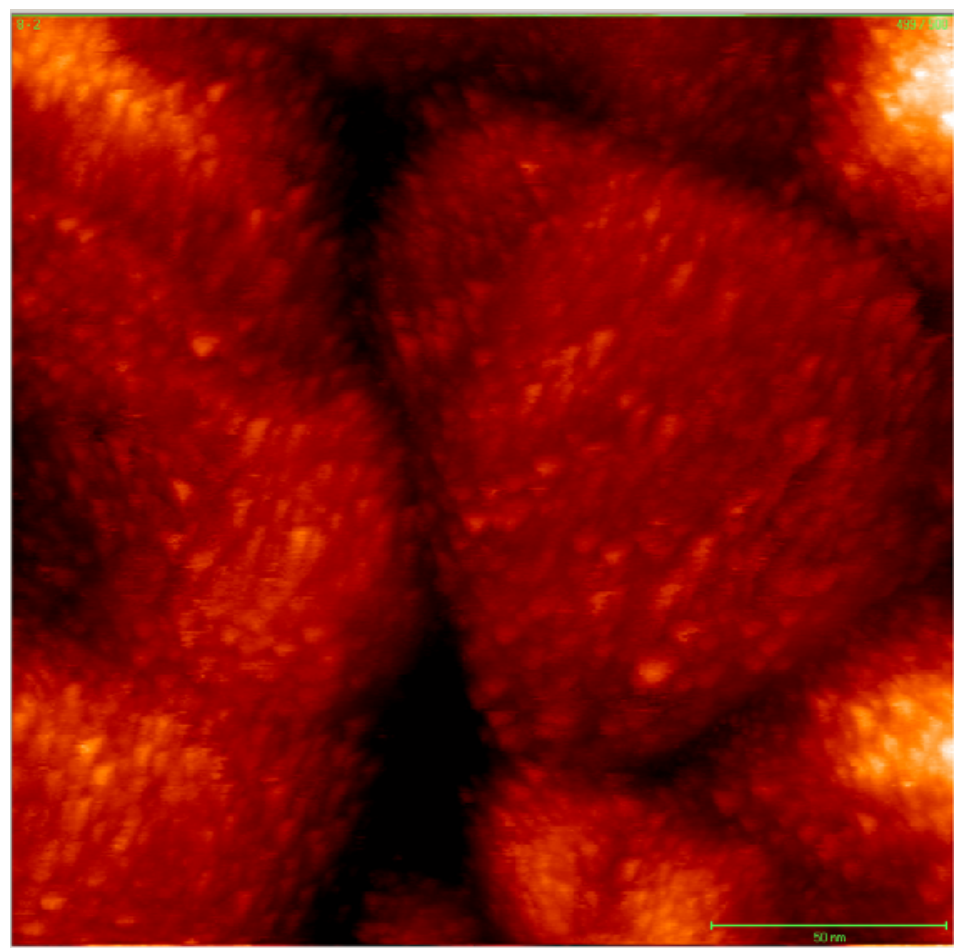

Fig. 5: STM image of Ti nanoclusters deposited on a Au/Si substrate. The green bar in the bottom right corner corresponds to $50 \mathrm{~nm}$.

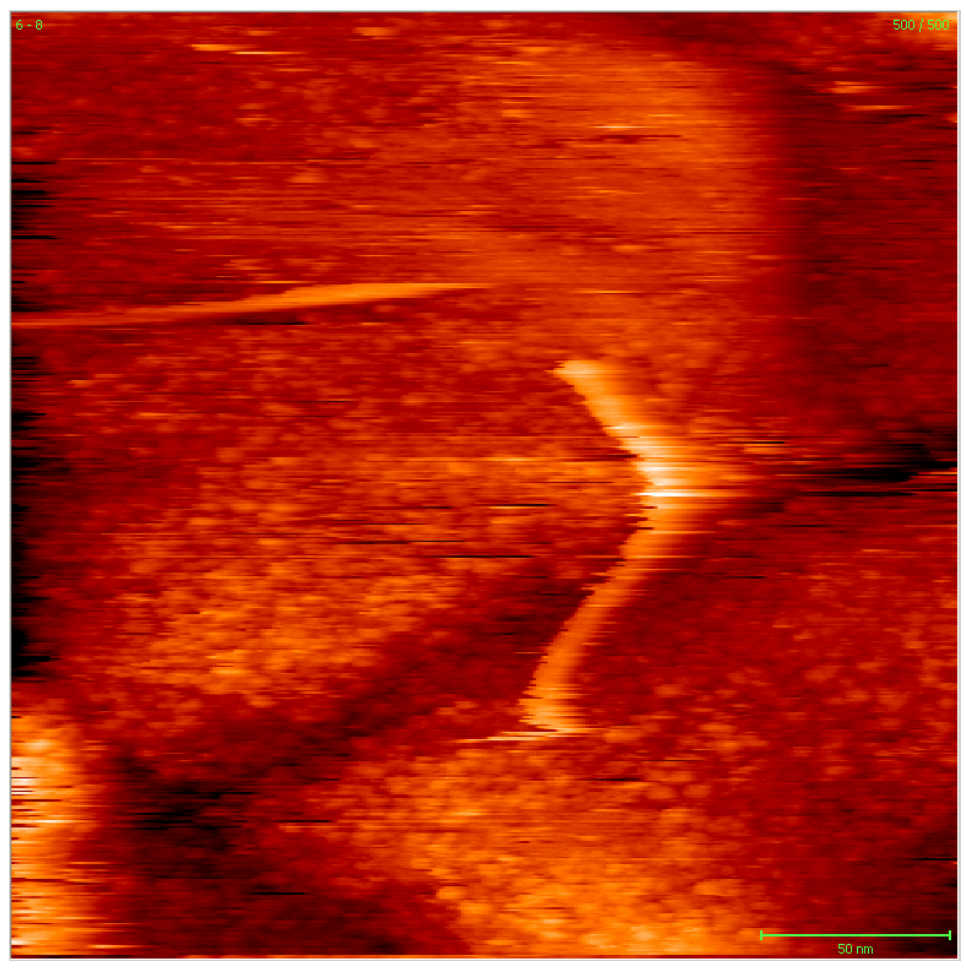

Fig. 6: STM image of an individual SWCNT with adsorbed Ti nanoclusters on a Au/Si substrate. The green bar in the bottom right corner corresponds to $50 \mathrm{~nm}$. 


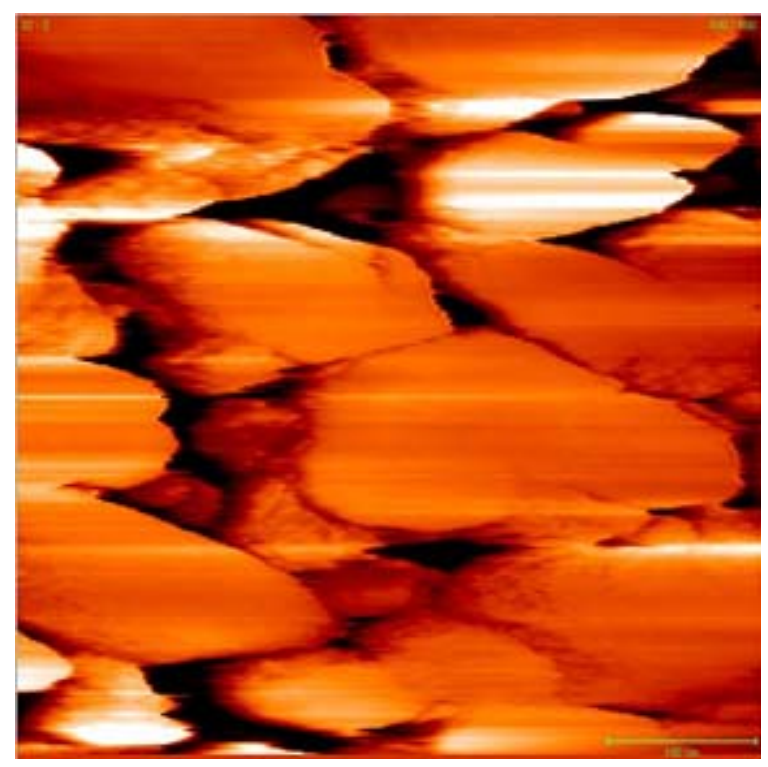

Fig. 7: STM image of Li/Au samples. The green scale bar is $100 \mathrm{~nm}$ long.

To allow an in-situ deposition of metals during scanning probe microscopy, we installed an EFM-3 e-beam evaporator in the Omicron VT-XA AFM (STM) microscope. Fig. 8 shows the schematic diagram (a) and experimental setup (b) for the in-situ metal deposition. The setup allows direct observation of structural transformation upon metal deposition on the atomic scale, as well as control and elucidation of the dynamic behavior as a function of deposition thickness, sample temperature, and other parameters.

In order to establish the working condition for the in-situ metal deposition experiments, we first performed the deposition of $\mathrm{Ti}$ on a Au substrate. The e-beam evaporator was degassed carefully so that the vacuum system was in the $10^{-10}$ mbar range during $\mathrm{Ti}$ deposition. The Ti deposition rate was controlled by an integrated flux monitor. For this experiment, Ti was deposited at a flux of $5 \mathrm{nA}$ for 3 seconds, which we expect to deliver Ti atoms on the sample surface in the submonolayer "decoration" regime. Fig. 9 shows the STM image of a Au sample before (a) and after (b) in-situ $\mathrm{Ti}$ deposition. It is evident that deposited $\mathrm{Ti}$ atoms form nanoparticles on the $\mathrm{Au}$ substrate particles. High-resolution images (Fig. 10a) show that these Ti nanoparticles have diameters in the range of $5 \mathrm{~nm}$, in good agreement with the previous deposition of $\mathrm{Ti}$ on $\mathrm{Au}$ substrates in a separate vacuum chamber. The advantage of the current in-situ experiment is the capability to monitor the evolution of the local electronic structure upon metal deposition, which is shown by the I(V) curve in Fig. 10b, measured directly on the Ti nanoparticle in Fig. 10a. 
(a)
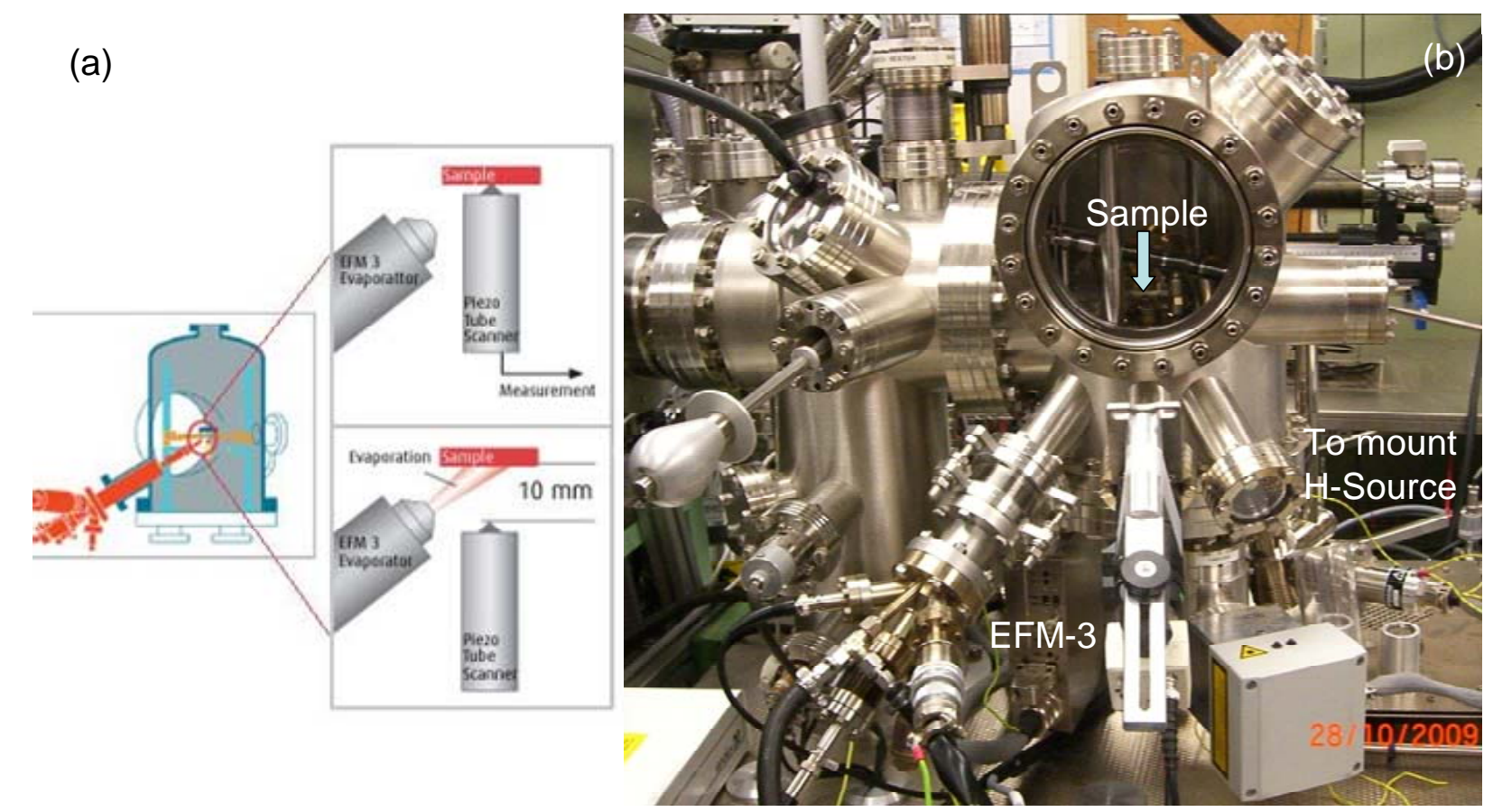

Fig. 8: Schematic diagram (a) and experimental setup (b) for an in-situ deposition of metals on sample surface in Omicron VT-AFM(STM).
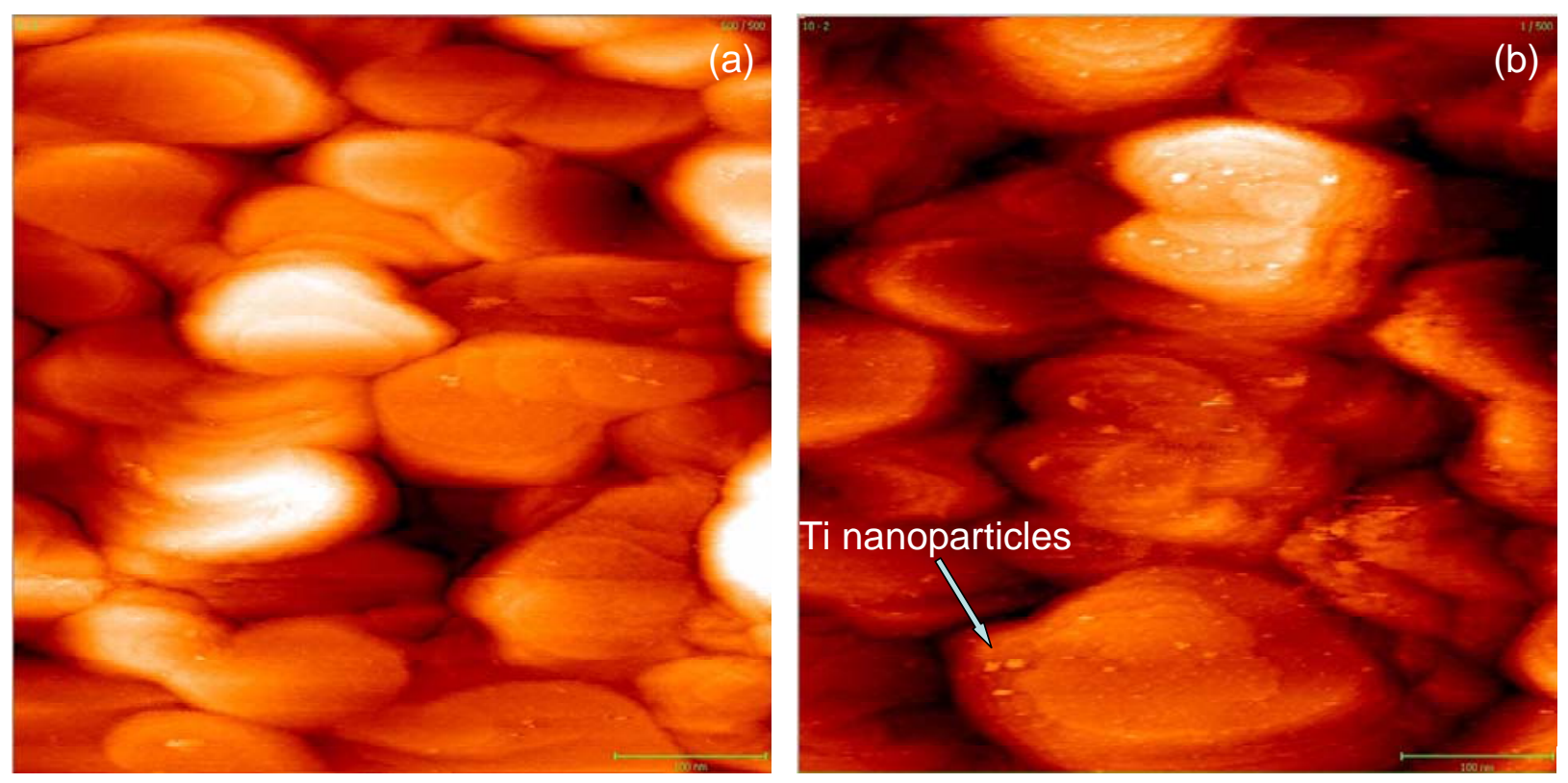

Fig. 9: Morphology of a Au substrate (100-200 nm particles) before (a) and after (b) in-situ Ti deposition (flux of $5 \mathrm{nA}$ for 3 seconds). Ti nanoparticles were formed on the surface of the $\mathrm{Au}$ substrate particles (see also high resolution image in Fig. 10). 


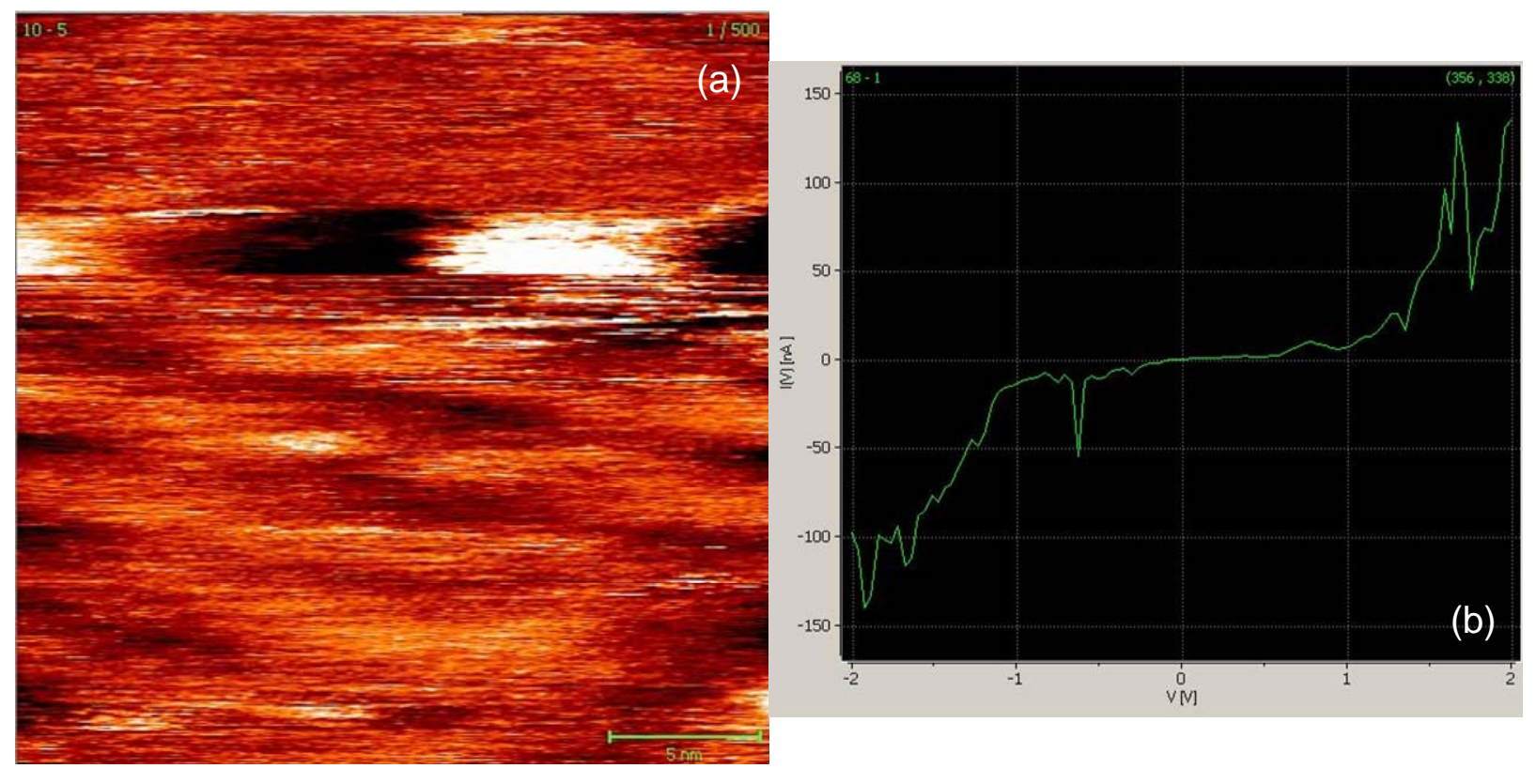

Fig. 10: (a) High-resolution STM image of a Ti nanoparticle ( $5 \mathrm{~nm}$ diameter) on a Au substrate; (b) I(V) curve obtained directly on the Ti nanoparticle.

Figs. 11 and 12 show further images of the in-situ STM experiments that allow us to locate and identify single clusters of deposited $\mathrm{Ti}$ atoms on the Au substrate. Note that the atomic layer structure of the Au substrate appears evident in the image, but that the Ti cluster(s) cannot be imaged on an atomic scale. This could be related to the different nature (electronic structure) of the Ti clusters and the substrate. In order to achieve better resolution for the Ti cluster, a finetuning of the parameters for STM imaging, such as bias voltage and tunneling current, is needed. Nevertheless, the Ti clusters observed on the Au substrate are again approx. $5 \mathrm{~nm}$ in size. The Ti clusters are often triangular in shape, which could result from the formation of $\mathrm{Ti}$ oxide nanocrystals. Local spectroscopy (STS, Fig. 12b), taken at the marked point on the Ti cluster in Fig. 12a), shows semiconducting (and not metallic) behavior. To further confirm the chemical composition of the Ti clusters, we have performed XPS on the same sample (note that the sample was transferred under UHV to the XPS chamber). The Ti 2p spectrum of the in-situ deposited $\mathrm{Ti} / \mathrm{Au}$ sample is shown in Fig. 12c, which clearly indicates the formation of $\mathrm{TiO}_{2}$. The results, again, confirm our observations that the deposited Ti metal is easily oxidized, even under UHV conditions (see, also, discussion below). Such behavior may have great impacts on the hydrogen storage capabilities of the Ti-decorated carbon nanomaterials. 


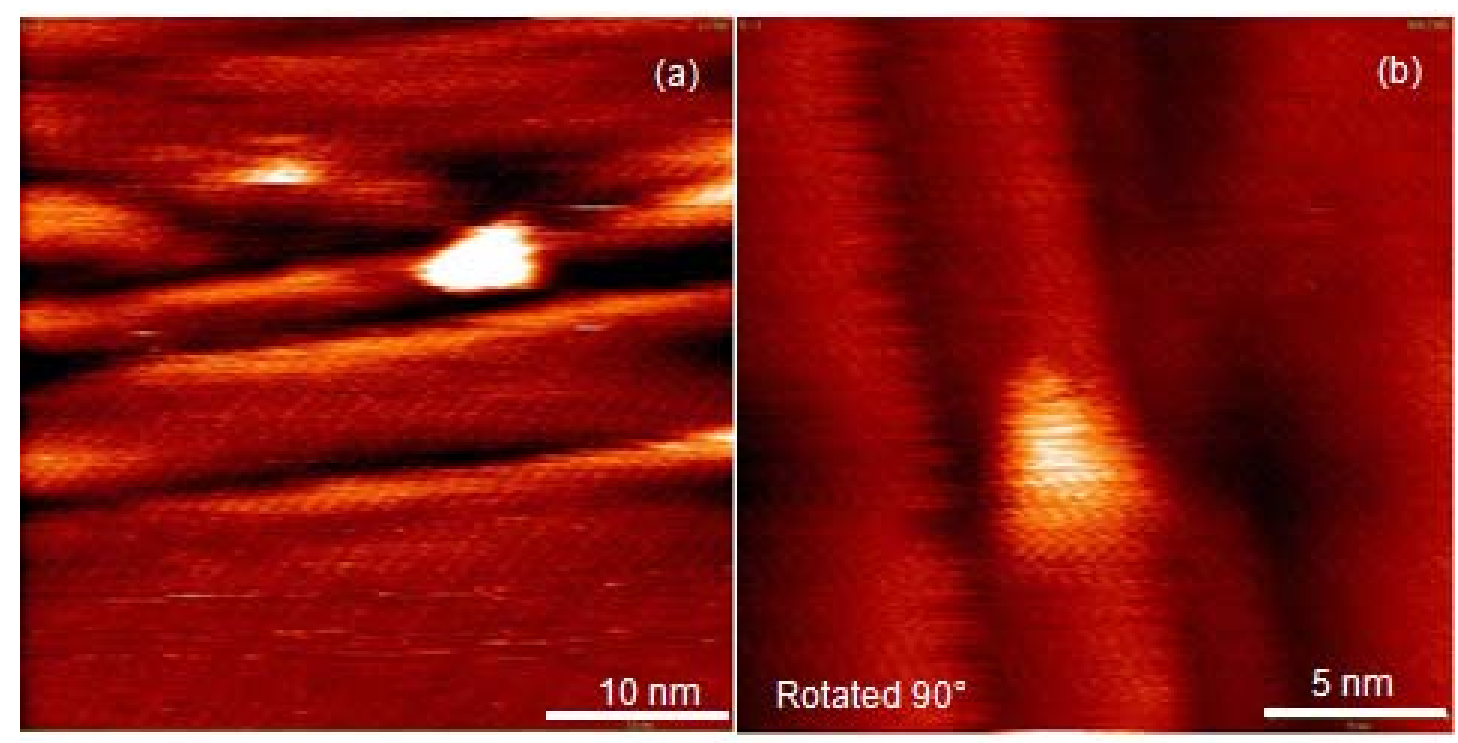

Fig. 11: STM images of an individual cluster of Ti after in-situ deposition on a Au substrate. The Ti cluster has a size of $\sim 5 \mathrm{~nm}$ and a triangular shape.
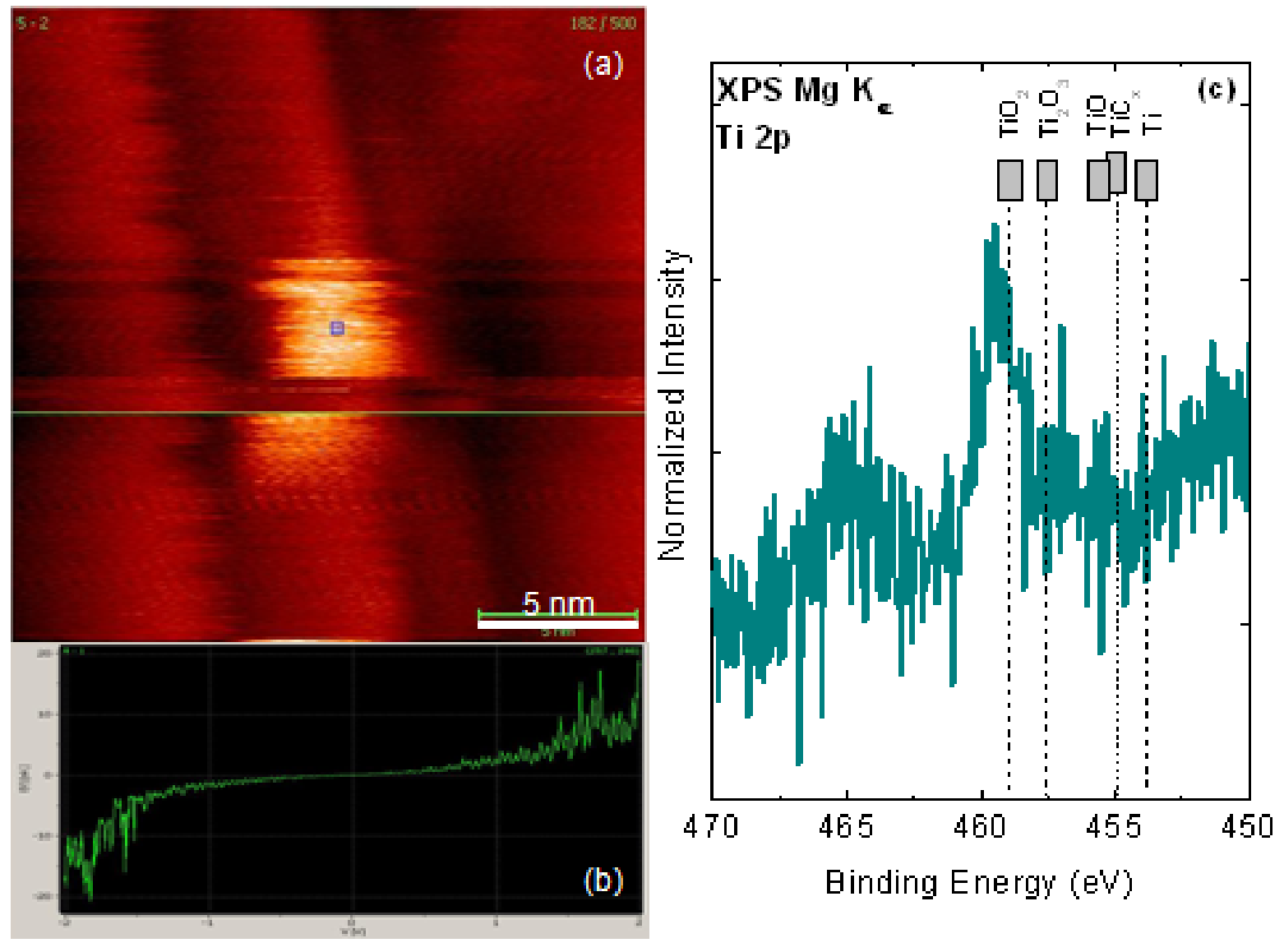

Fig. 12: (a) Morphology of an individual Ti cluster (STM); (b) Local scanning tunneling spectroscopy (STS) at the marked point on the Ti cluster in (a); (c) XPS Ti 2p spectrum of the insitu Ti-deposited Au sample, which shows the presence of $\mathrm{TiO}_{2}$. 

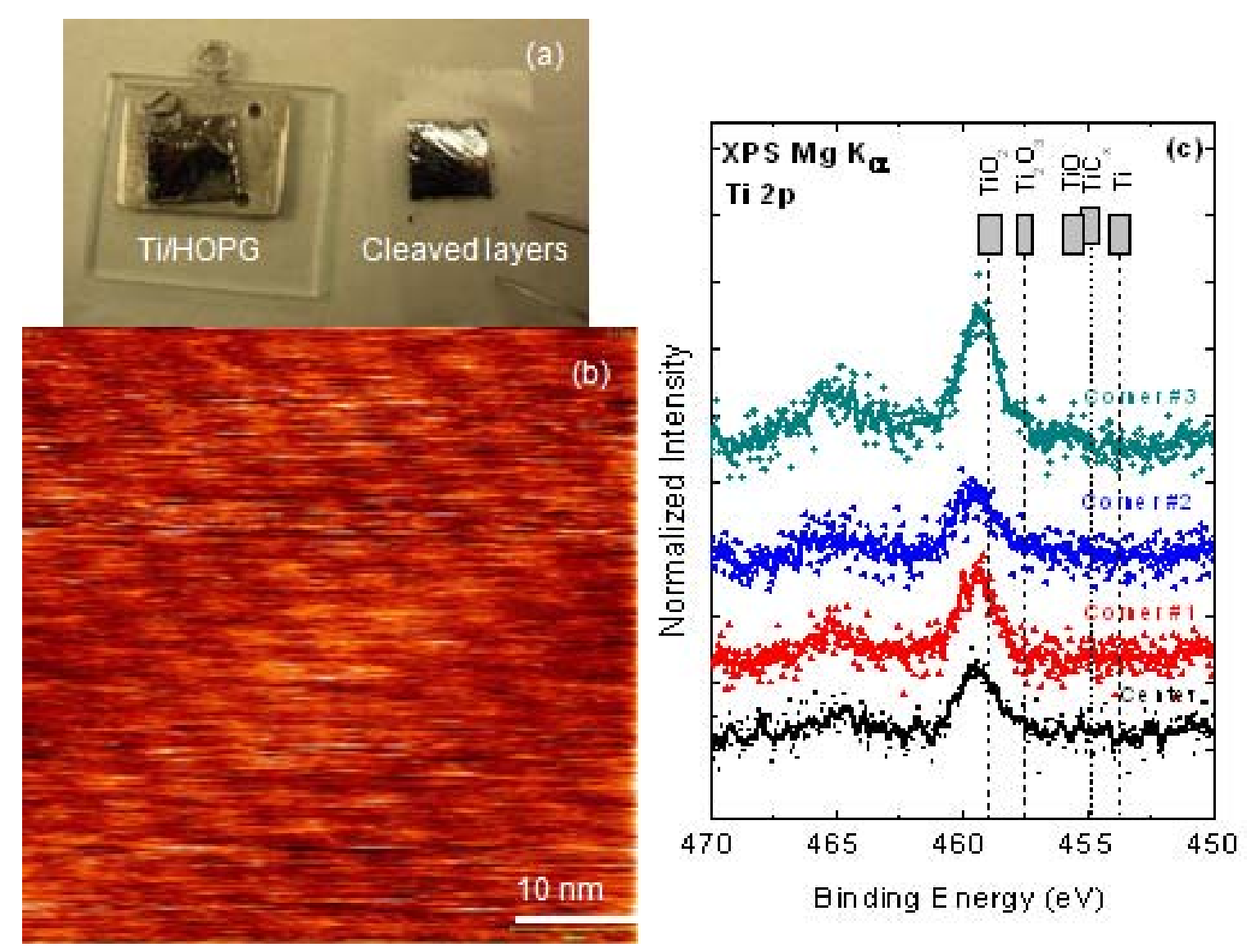

Fig. 13: (a) Cleaving of the previously Ti-deposited HOPG sample; (b) STM shows unusual periodic and "wave-like" structure at large scale; (c) XPS Ti 2p spectra at the center and corners of the sample indicate the presence of $\mathrm{Ti}$ in the form of $\mathrm{TiO}_{2}$ on the cleaved sample.

Finally, we present a microscopy (STM) and spectroscopy (XPS) study of Ti atoms deposited on a HOPG sample. The deposited Ti atoms form clusters on the surface of HOPG, and the Ti clusters are also oxidized under UHV. We then took the Ti-deposited HOPG sample outside of the vacuum chamber and stored it in air. In an attempt to re-use this HOPG sample for STM experiments, we freshly cleaved the sample in a glove box (under $\mathrm{N}_{2}$ gas) using scotch tape (Fig. 13a). STM experiments on this sample show an unusual periodic (and "wave-like") structure (Fig. 13b), which is completely different from the periodic structure (at much smaller scale) of the atomic arrangement of carbon atoms in HOPG. We then performed XPS measurements on the cleaved (previously Ti deposited) HOPG sample, and unexpectedly found the presence of $\mathrm{TiO}_{2}$ on the sample (Fig. 13c). Using XPS in small area mode, we found that the presence of $\mathrm{Ti}$ in the form of $\mathrm{TiO}_{2}$ is relatively uniform throughout the sample surface (spectra were taken at the center and corners of the sample). The presence of Ti on the freshly cleaved HOPG sample thus indicates that $\mathrm{Ti}$ (or $\mathrm{TiO}_{2}$ ?) has diffused deeply into the HOPG sample. The diffusion of Ti at this length scale (after a careful removal of tens of layers of graphite) is unprecedented and unexpected. To confirm this diffusion behavior, we have cleaved the HOPG sample one more time. XPS measurements (not shown) again confirm the presence of $\mathrm{Ti}$, in the form of $\mathrm{TiO}_{2}$, on the surface of the (twice) cleaved HOPG sample. STM experiments on this sample show several 
interesting features of the surface morphology, such as the accumulation of $\mathrm{TiO}_{2}$ clusters along the terraces of HOPG (Fig. 14a), triangular-shape clusters (presumably of $\mathrm{TiO}_{2}$ nanocrystals, Fig. 14b), a Ti cluster and a hole left behind after cleaving the HOPG surface (Fig. 14c) and, again, an unusual periodic structure of the HOPG surface (Fig. 14d). These multi-form behaviors of Ti clusters after diffusing at an unprecedented length scale into HOPG will require further studies to provide insight into the formation and diffusion of $\mathrm{Ti}$ into carbon nanomaterials.
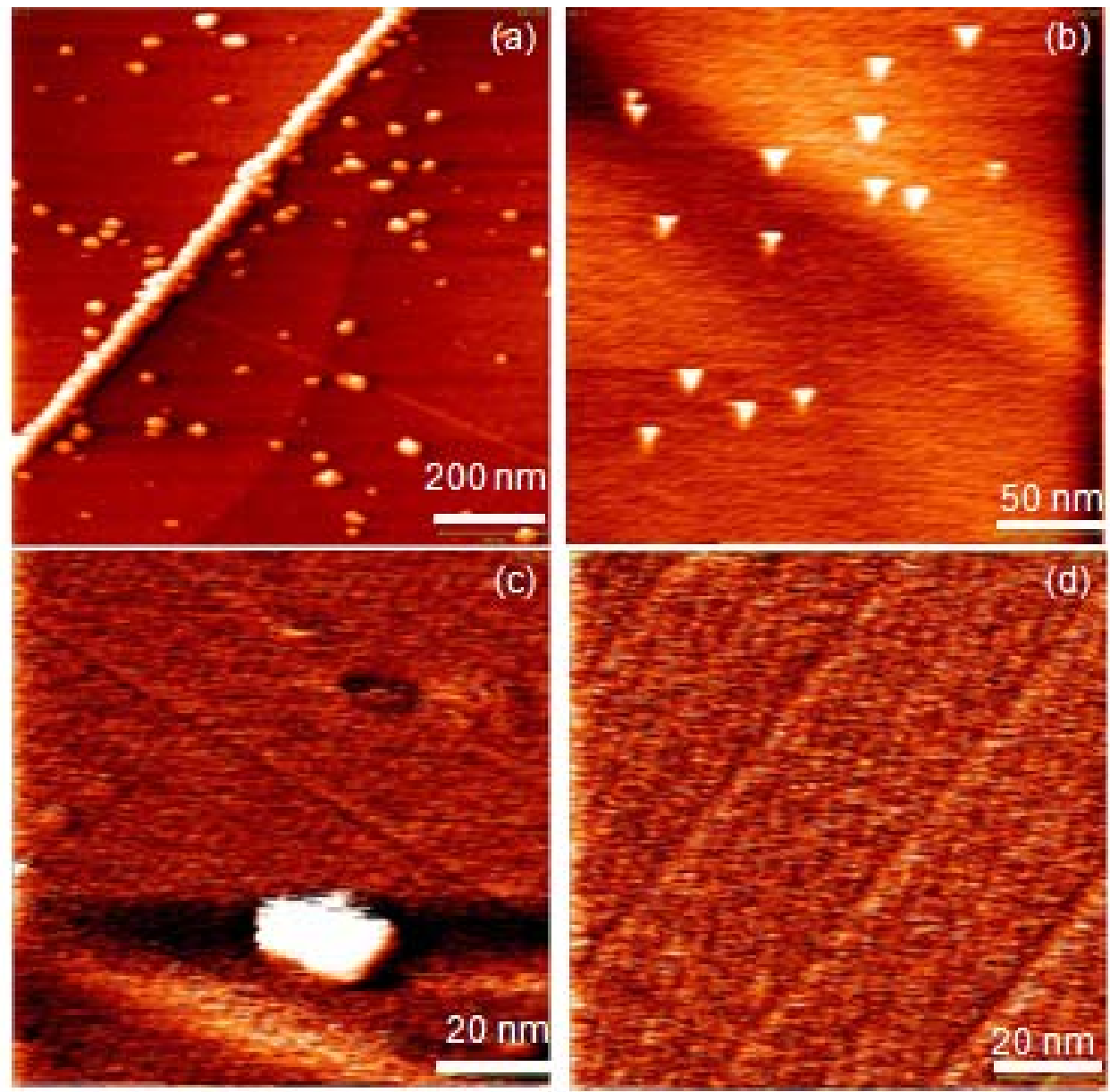

Fig. 14: Multi-form morphology of an HOPG surface and of Ti clusters after diffusing deeply into the HOPG sample: (a) Accumulation along terraces of the HOPG surface; (b) triangular shape of Ti clusters; (c) a Ti cluster and hole on the HOPG surface; (d) unusual periodic structure of HOPG surface. 


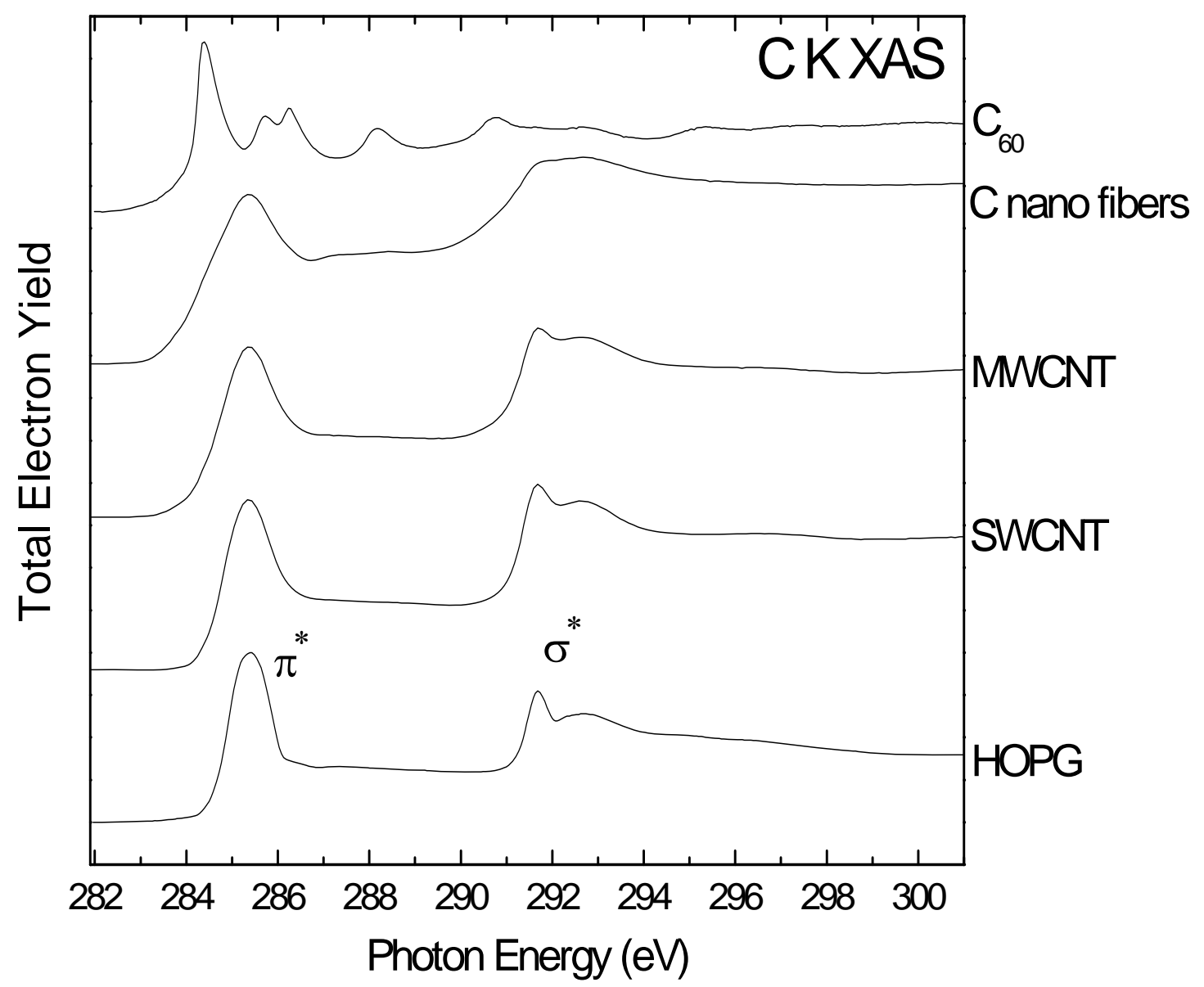

Fig. 15: X-ray absorption spectra of different nanomaterials and a HOPG reference.

\section{Soft x-ray spectroscopy at the Advanced Light Source, Lawrence Berkeley National} Laboratory

Throughout our project, $\mathrm{x}$-ray absorption and x-ray emission spectra of carbon nanomaterials were collected at the Advanced Light Source, Lawrence Berkeley National Laboratory. Fig. 15 shows the x-ray absorption spectra of different carbon nanomaterials, compared with a HOPG reference. All spectra show similar spectral features for their $\sigma^{*}$ and $\pi^{*}$ systems. The only exception is the spectrum of $\mathrm{C}_{60}$, where sharper resonances are observed, as expected for a molecule. A first series of $x$-ray emission spectra of one selected carbon nanomaterial $\left(C_{60}\right)$ is shown in Fig. 16. Together with theoretical calculations performed by the group of $\mathrm{C}$. Chen at UNLV (Fig. 16, right), a detailed picture of the occupied electronic states of the samples can be obtained. 

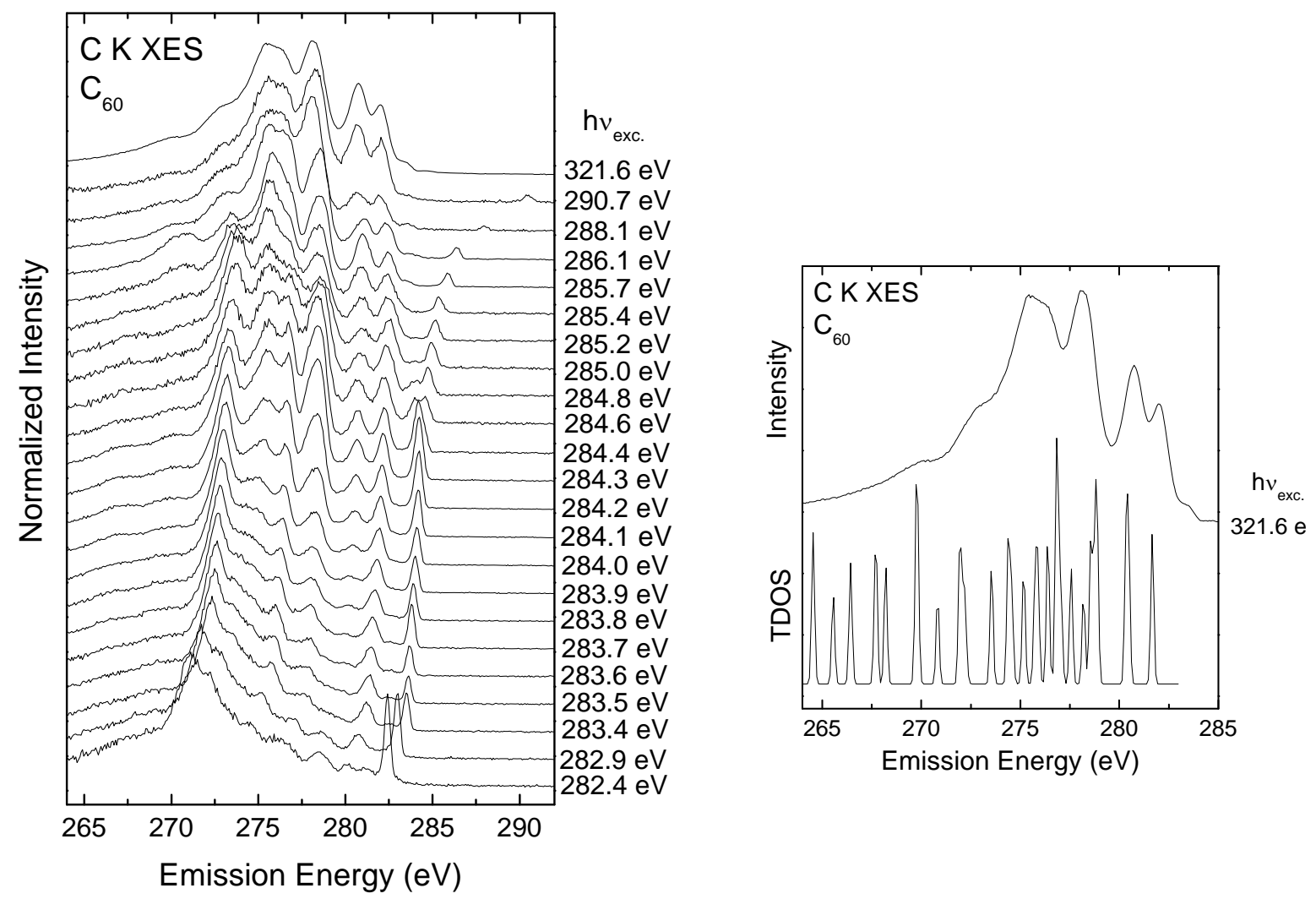

Fig. 16: Resonant $\mathrm{C} \mathrm{K} \mathrm{x-ray} \mathrm{emission} \mathrm{series} \mathrm{of} \mathrm{C}_{60}$ (left) and comparison between the nonresonant $\mathrm{X}$-ray emission spectrum and the density of states calculated by density functional theory in the group of C. Chen, UNLV Department of Physics.

Recently, it became possible to investigate the electronic structure of carbon nanomaterials in a so-called "RIXS map" approach. Fig. 17 shows such a resonant x-ray emission measurement. The software for acquiring such maps was written by Lothar Weinhardt, the post-doc overseeing the FCAST activities of our task at that time. Such maps represent a real advancement in X-ray emission spectroscopy (XES) and have previously not been thought of due to transmission and resolution limitations of the soft x-ray spectrometers. The maps give unprecedented detailed insight into the electronic structure of the investigated materials. 


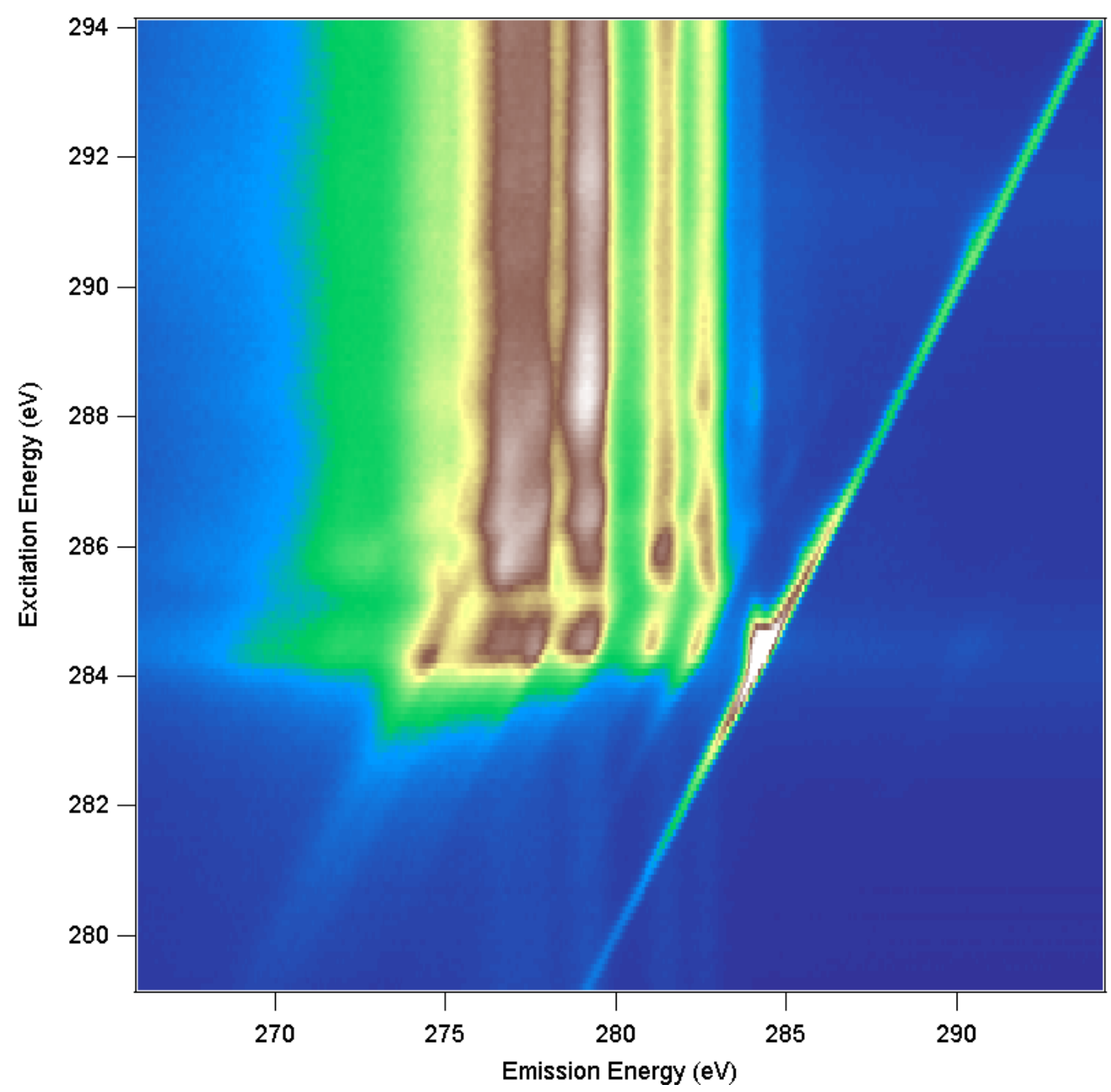

Fig. 17: X-ray emission map of $\mathrm{C}_{60}$. Emission and Excitation photon energies are shown on the $\mathrm{x}$ - and $\mathrm{y}$ - axis, respectively. Intensities are color-coded (white: high intensity, blue: low intensity). The map has a very rich structure containing detailed information about the electronic structure of $\mathrm{C}_{60}$.

3. X-ray photoelectron spectroscopy study of interactions between hydrogen, carbon nanomaterials, and/or metals

To study the interaction between metal adatoms and carbon nanomaterials prior to hydrogen loading, we have prepared and characterized carbon nanomaterials functionalized with Ti. Several theoretical groups predict that such a functionalization will increase the hydrogen storage capacity of single walled carbon nanotubes (SWCNT).

A series of different samples were prepared and measured with surface-sensitive techniques in our lab at UNLV (and with bulk-sensitive methods at the Advanced Light Source). Three sets of 


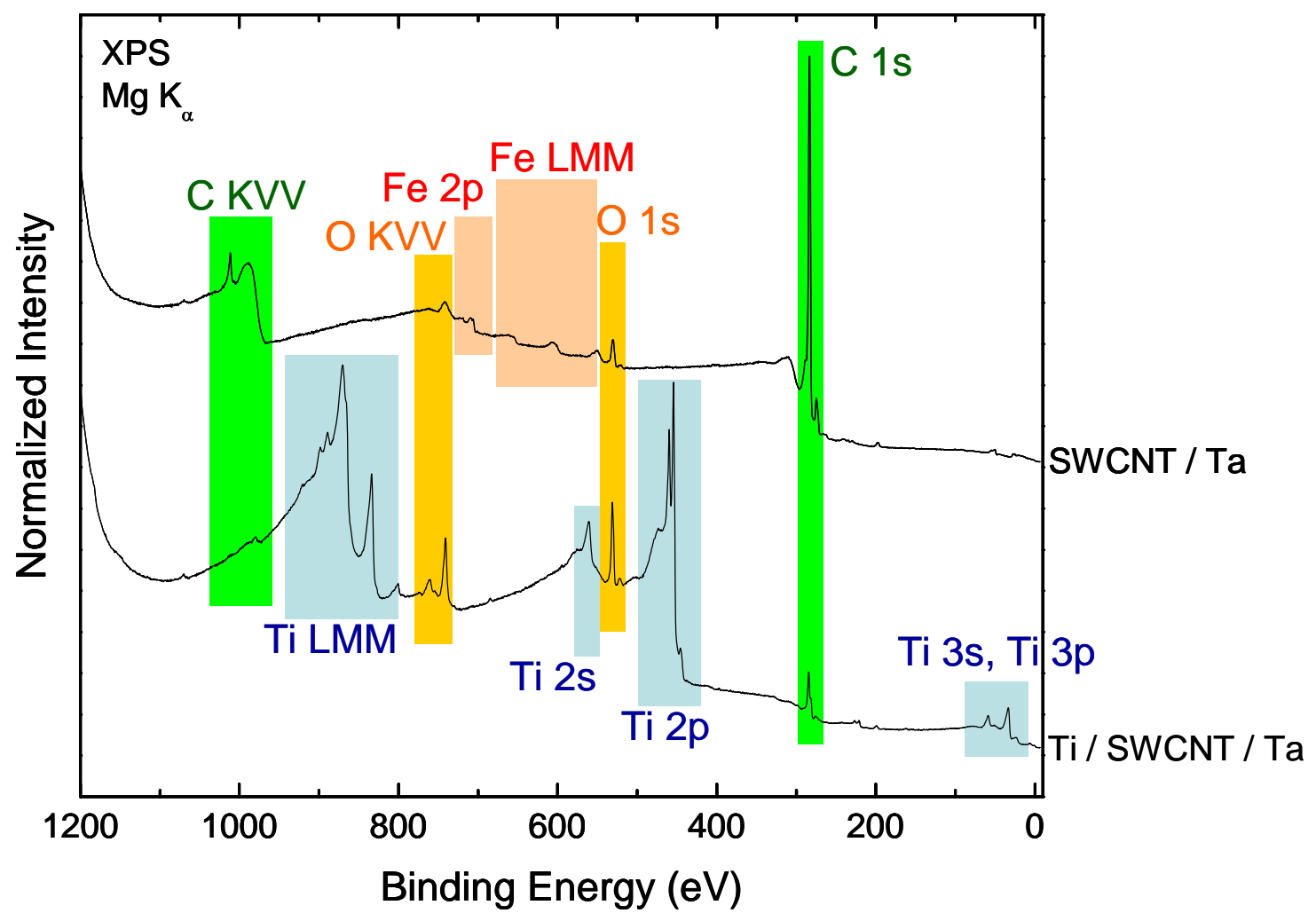

Fig. 18: XPS survey spectra of SWCNT powder pressed into Ta foil with and without a deposited Ti layer with a thickness of approx. $2 \mathrm{~nm}$.

samples, each set consisting of SWCNT, Bucky Paper prepared from SWCNT, and $\mathrm{C}_{60}$, were prepared by pressing the corresponding powders into Indium or Tantalum foils. One set was investigated without any further preparation, the second after deposition of about $2 \mathrm{~nm}$ of Ti by thermal evaporation, and the third after $2 \mathrm{~nm}$ Ti deposition and subsequent heating of the sample. All nine samples were investigated by X-ray photoelectron spectroscopy (XPS), X-ray absorption spectroscopy (XAS), and X-ray emission spectroscopy (XES), and the XPS results are discussed here.

In Fig. 18, the XPS survey spectra of a SWCNT sample pressed in Ta foil with and without deposited $\mathrm{Ti}$ are shown. The absence of any Ta lines indicates that the Ta substrate was fully covered with SWCNT powder. Besides the expected C lines, we find some $\mathrm{O}$ adsorbates at the sample surface, which naturally occur even after short exposure to air. In addition we can identify Fe lines, which are due to the catalyst used during the production process of the SWCNT. 


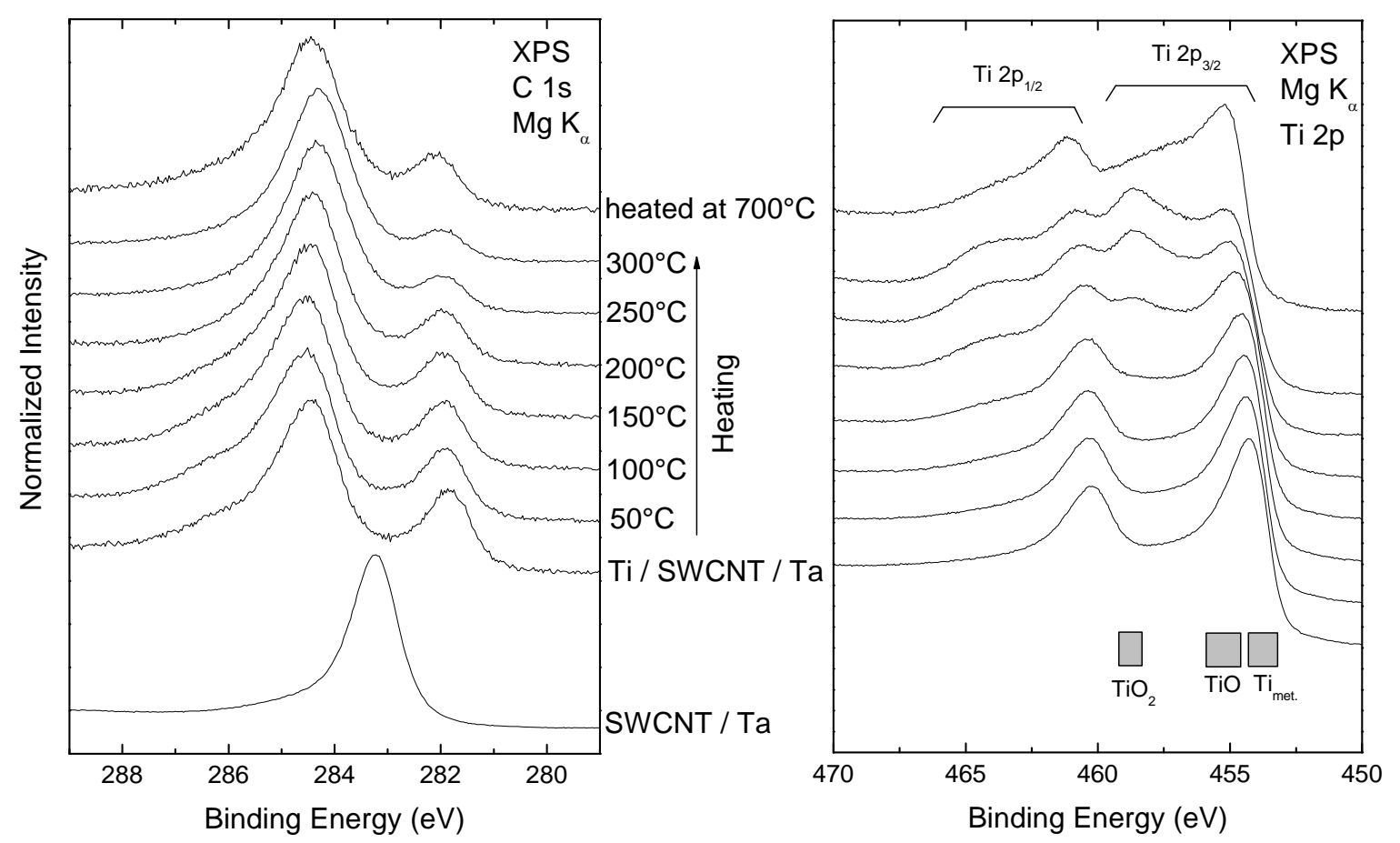

Fig. 19: XPS C 1s (left) and Ti 2p (right) detail spectra of SWCNT/Ta and Ti/SWCNT/Ta after subsequent heating steps ( 1 hour each) at increasing temperatures $\left(50^{\circ} \mathrm{C}-300^{\circ} \mathrm{C}\right)$, as well as a Ti/SWCNT/Ta sample heated for 1 hour at $700^{\circ} \mathrm{C}$. The rectangles in the right figure indicate the $\mathrm{Ti} 2 \mathrm{p}_{3 / 2}$ line positions of reference samples found in the literature (the horizontal width corresponds to the spread of these values). All spectra were normalized to the respective maximum.

After the deposition of $\mathrm{Ti}$, all expected $\mathrm{Ti}$ lines emerge in the survey spectrum. In parallel, the $\mathrm{C}$ lines are strongly attenuated by the Ti layer. Furthermore, the intensity of the $\mathrm{O}$ 1s line strongly increases. This can be explained by the fact that the Ti surface is very reactive and is rapidly covered by adsorbates even under ultra-high vacuum (UHV) conditions. In fact, Ti-covered surfaces are commonly used as pumps for UHV applications by renewing the Ti layer every few hours.

The finding of a very reactive Ti layer is supported by the Ti 2p detail spectra presented in Fig. 19, right. By comparing with literature values, the Ti compound found shortly after Ti deposition already exhibits a line shape that is indicative of a partial oxidation (TiO). When subsequently heating the sample, a second chemical Ti species emerges, identified as $\mathrm{TiO}_{2}$, indicating an increasing oxidation of the Ti film. Comparison with a sample which was only heated once (but at a much higher temperature, top spectrum) suggests that the actual temperature during the heating is of less importance than the total exposure time of the surface.

The C 1s detail spectra presented in Fig. 19, left, reveal dramatic changes after the deposition of Ti: several different (at least three) $\mathrm{C}$ species can be identified. At the position of the $\mathrm{C} 1 \mathrm{~s}$ line in 
the pure SWCNT/Ta sample, no peak is found after Ti deposition. This indicates a strong interaction between $\mathrm{Ti}$ and the vast majority (if not all) of the $\mathrm{C}$ atoms in the SWCNT. Furthermore, some carbon emission can presumably be attributed to adsorbates on the Ti surface.

To investigate the interaction of both, molecular and atomic hydrogen with nanomaterials (in particular with metal-decorated and functionalized SWCNTs), an atomic hydrogen source was built and commissioned. Fig. 20 shows a photograph of the source. The central part is a W capillary that can be heated to more than $1800^{\circ} \mathrm{C}$ by electron bombardment from a filament. The temperature of the capillary is controlled with a thermocouple directly attached to the capillary. Hydrogen is introduced into this capillary via a leak valve used to control the hydrogen flow. If the capillary is at sufficient temperature $\left(>1600^{\circ} \mathrm{C}\right)$ the hydrogen molecules are "cracked" and a directed beam of atomic hydrogen is achieved.

The results of a first hydrogenation step of single-walled carbon nanotubes (SWCNT) using our atomic hydrogen source are shown in Fig. 21. These experiments were performed to serve as a baseline when studying the hydrogen adsorption characteristics of metal-decorated SWCNT and other nanomaterials. The adsorption of atomic hydrogen was studied in parallel to the adsorption of molecular hydrogen. Since the spectroscopic signature of the hydrogen-nanomaterial interaction is expected to be strongest in the case of atomic hydrogen, larger initial emphasis was placed on this approach. Furthermore, it is conceivable that catalytic approaches (including metal decoration) will lead to an atomic-like adsorption process even if the nanomaterial is exposed to molecular hydrogen.

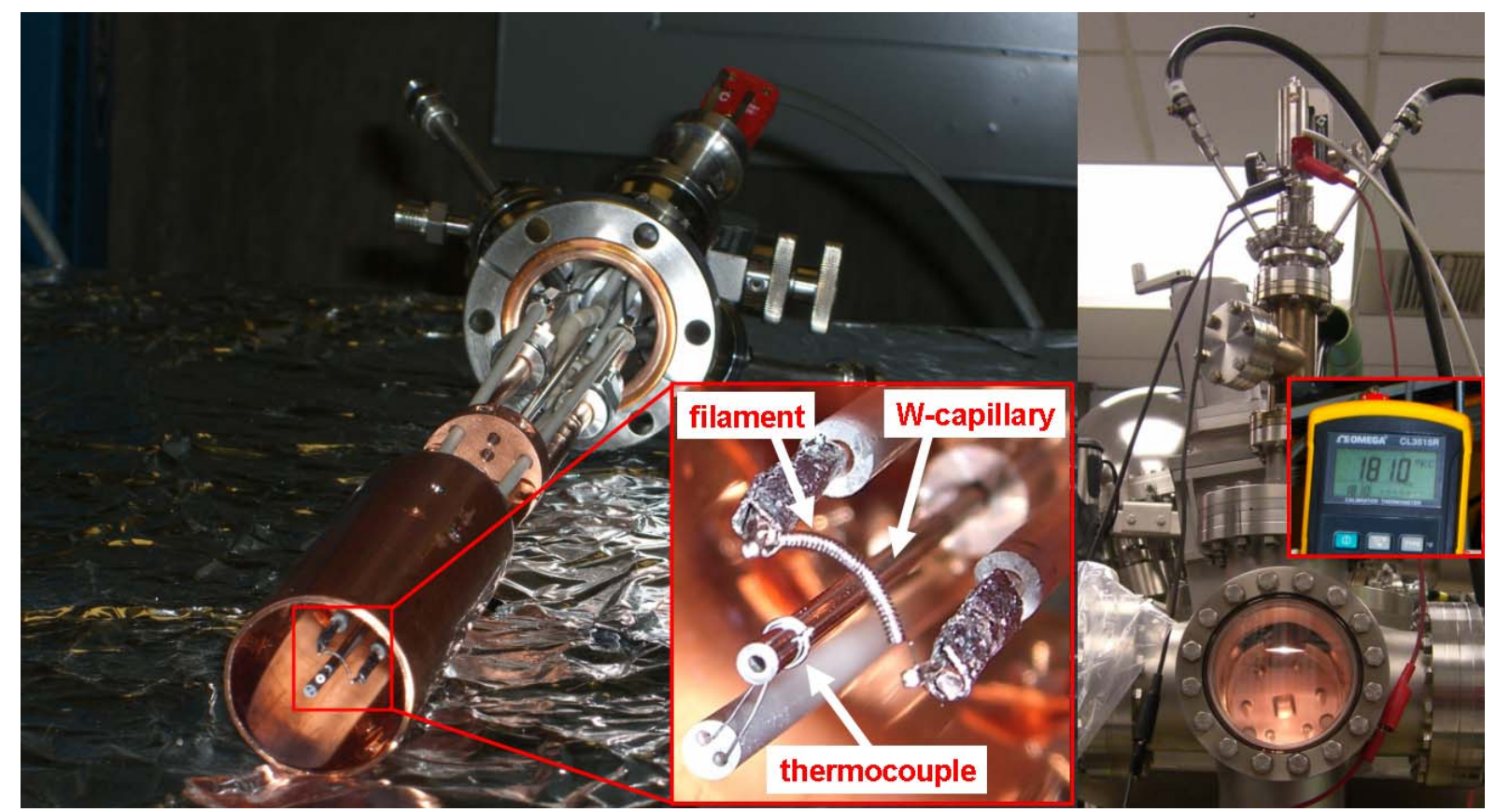

Fig. 20: Left: Atomic hydrogen source. The inset shows the assembly of capillary, filament, and thermocouple. Right: Atomic Hydrogen Source in operation, the actual temperature (1810 ${ }^{\circ} \mathrm{C}$ ) is shown in the inset. 

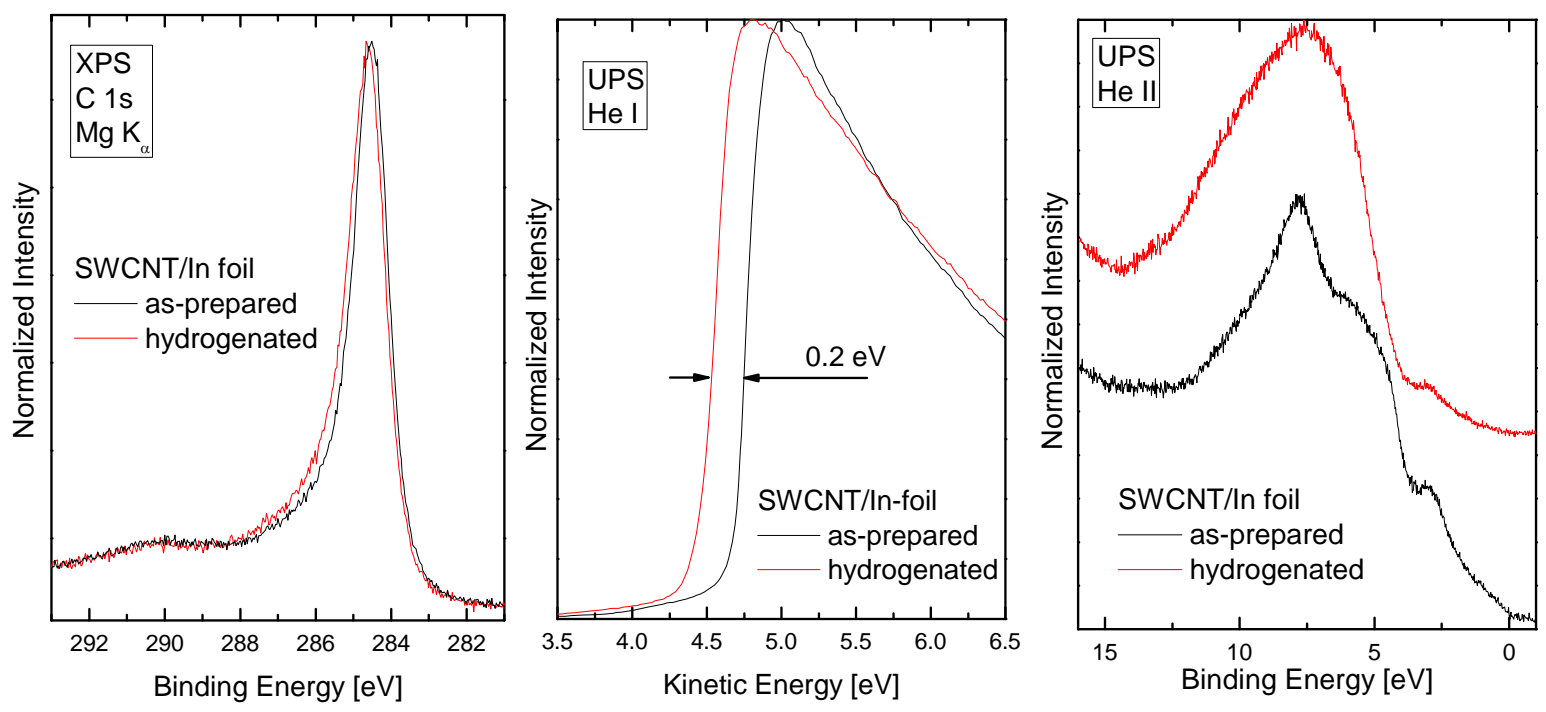

Fig. 21: SWCNT/In foil before (black) and after hydrogenation (red): XPS C 1s spectrum (left), secondary electron cutoff (center), valence band (right).

Adsorption of atomic hydrogen on SWCNTs leads to a second, chemically different carbon species, as is evident from a second component on the high binding energy side of the $\mathrm{C} 1 \mathrm{~s}$ photoemission spectrum. In the spectra presented in Fig. 21 (left) this additional component is visible as a slightly increased intensity on the high binding energy side of the peak. In addition to the chemical shift in the $\mathrm{C} 1 \mathrm{~s}$ line, we also find a shift of the work function by $0.2 \mathrm{eV}$, as shown by the secondary electron cutoff in Fig. 21 (center) of our UPS measurements. The valence band spectrum shown in Fig. 21 (right) also shows strong changes upon hydrogenation. These changes can be correlated to a change in hybridization of the $\mathrm{C}$ atoms from $\mathrm{sp}^{2}$ to $\mathrm{sp}^{3}$ bonding during the absorption of hydrogen, which leads to a change of the electronic valence structure.

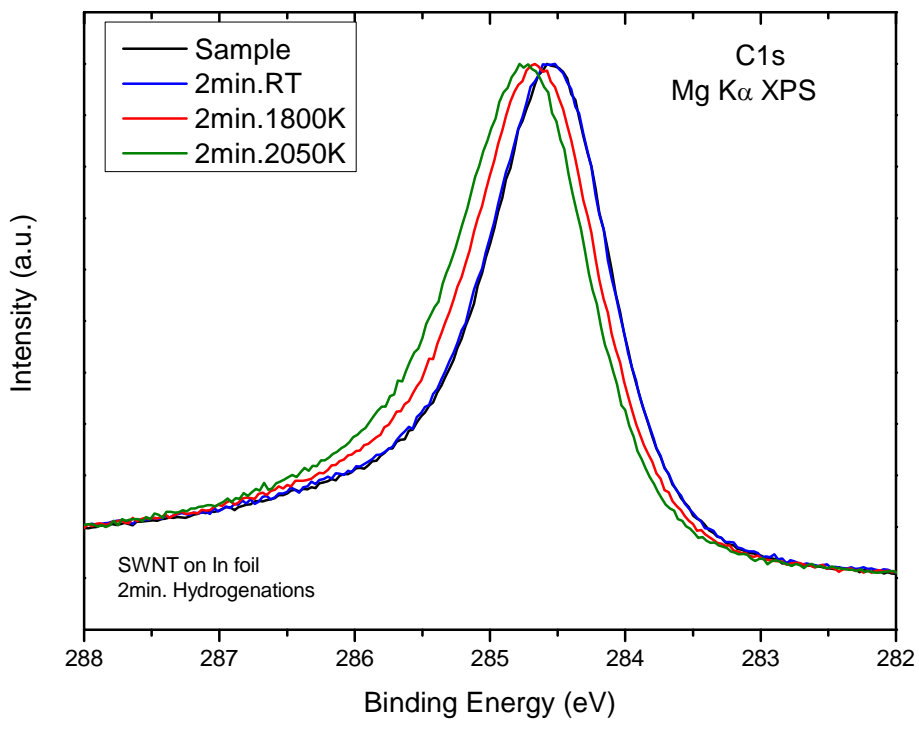

Fig. 22: C 1s XPS spectra showing hydrogenation of SWCNTs. 


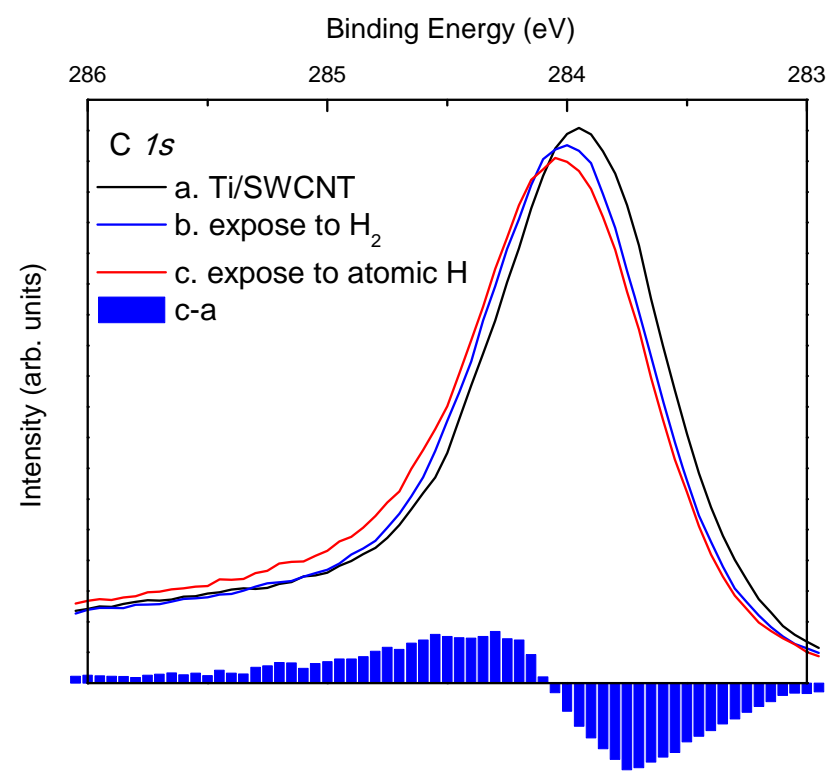

Fig. 23: C 1s XPS spectra showing hydrogenation of Ti-decorated SWCNTs.

Fig. 22 shows a series of C 1s X-ray photoelectron spectroscopy (XPS) spectra of SWCNTs after being exposed to hydrogen with different temperatures of the Hydrogen source. At room temperature, only molecular hydrogen is emitted from the source. The $\mathrm{C} 1 \mathrm{~s}$ spectrum shows no difference between the such-hydrogenated sample and the sample without hydrogen exposure. Therefore, the molecular hydrogen does not affect the chemical and electronic environment of carbon in SWCNTs. However, when the source temperature is above $1800 \mathrm{~K}$, atomic hydrogen is produced as well, and thus the $\mathrm{C} 1 \mathrm{~s}$ peak shifts to higher binding energy, most likely due to a charge transfer between adsorbed atomic hydrogen and the $\mathrm{C}$ atoms of the SWCNTs. Note that the ratio between atomic and molecular hydrogen emitted from the source depends on the source temperature. Consequently, the shift for the $2050 \mathrm{~K}$ hydrogenation is larger than for the $1800 \mathrm{~K}$ hydrogenation.

Fig. 23 shows a series of C 1s XPS spectra from Ti-decorated SWCNTs. This time, the C 1s peak exhibits a shift even for the sample that was exposed to molecular hydrogen (i.e., with the hydrogen source at room temperature). This indicates that the presence of Ti on the SWCNTs surface allow the adsorption of molecular hydrogen, contrary to the case without Ti. Since we find this change in the carbon core level, the adsorption of hydrogen apparently modifies the chemical and electronic environment of carbon in the samples.

A series of Ti 2p XPS spectra from the same set of samples is shown in Fig. 24. As can be easily seen, the electronic structure of $\mathrm{Ti}$ is also changed by exposing the Ti-decorated SWCNTs to hydrogen. The change is especially pronounced after being exposed to atomic hydrogen, as expected, but also the exposure to molecular hydrogen leads to a significant change. For adsorption of atomic hydrogen, a new feature appears in the spectrum (at approx. $455 \mathrm{eV}$ ), which 


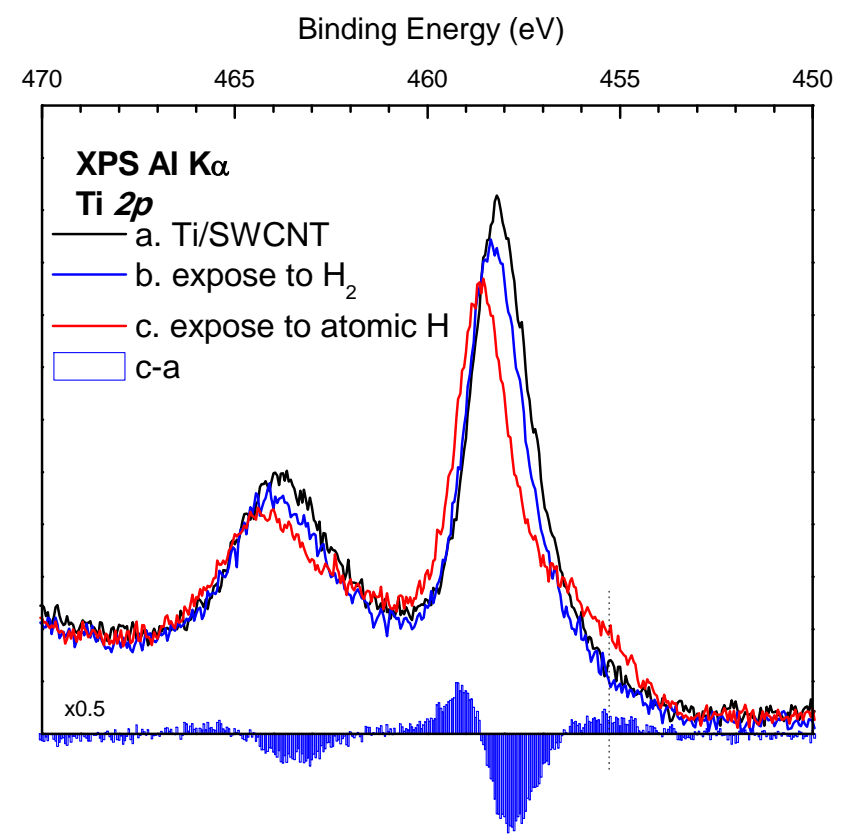

Fig. 24: Ti 2p XPS spectra showing hydrogenation of Ti-decorated SWCNTs.

indicates a new chemical environment for $\mathrm{Ti}$ atoms. Since we only find this feature after atomic hydrogen exposure, we propose that this feature can be used to monitor dissociation processes on the surface.

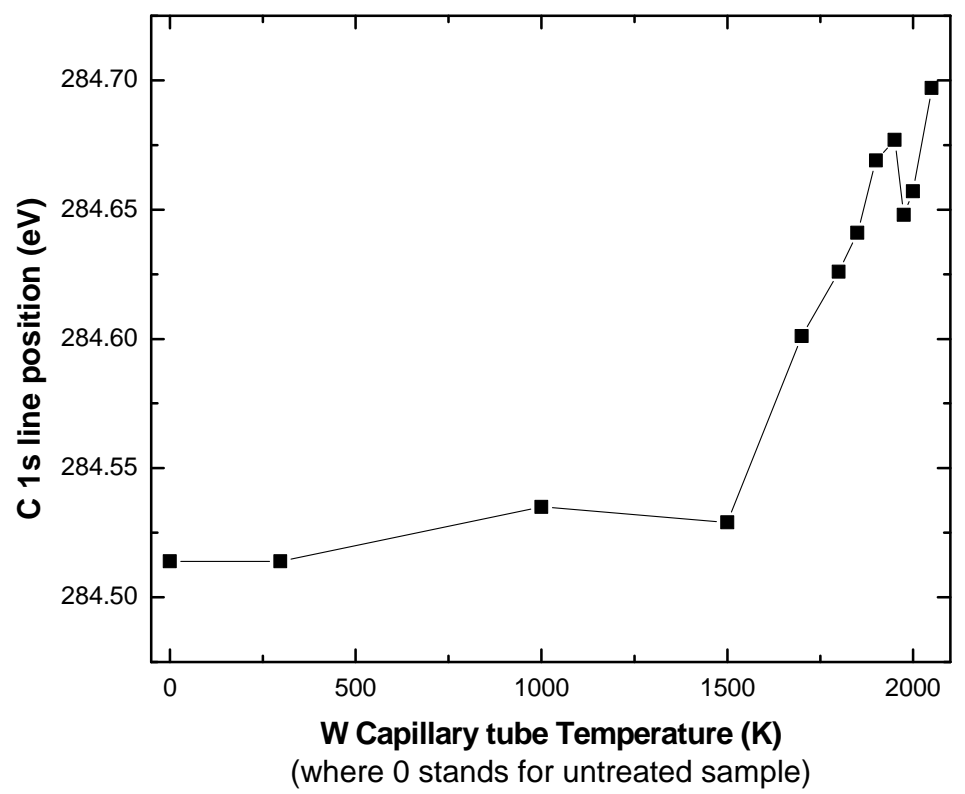

Fig. 25: SWNT C 1s core level position as a function of hydrogenation treatment temperature. 
Fig. 25 shows a change of $\mathrm{C}$ 1s core level energy position for SWNTs hydrogenated at different atomic hydrogen source temperatures. The ratio between atomic and molecular hydrogen emitted from the source increases with the source temperature. For source temperatures below $1500 \mathrm{~K}$, the $\mathrm{C}$ 1s peak position is constant. In contrast, the peak shifts towards higher binding energy for source temperatures above $1500 \mathrm{~K}$. The increased fraction of atomic hydrogen in the hydrogen beam clearly induces a shift of the $\mathrm{C} 1 \mathrm{~s}$ core level. Furthermore (not shown), we observe a broadening of the $\mathrm{C} 1 \mathrm{~s}$ line.

Fig. 26 shows valence band structures of SWNTs hydrogenated at different source temperatures. The feature at $3 \mathrm{eV}$ indicates the degree of $\pi$-conjugation of the $\mathrm{sp}^{2}$-hybridized carbon network of SWNTs. The decrease of its spectral intensity with increasing source temperature is likely due to a change of the $\mathrm{C}$ hybridization state (from $\mathrm{sp}^{2}$ to $\mathrm{sp}^{3}$ ) in SWNTs after adsorption of hydrogen. This observation is consistent with our findings from the C 1s XPS measurements.

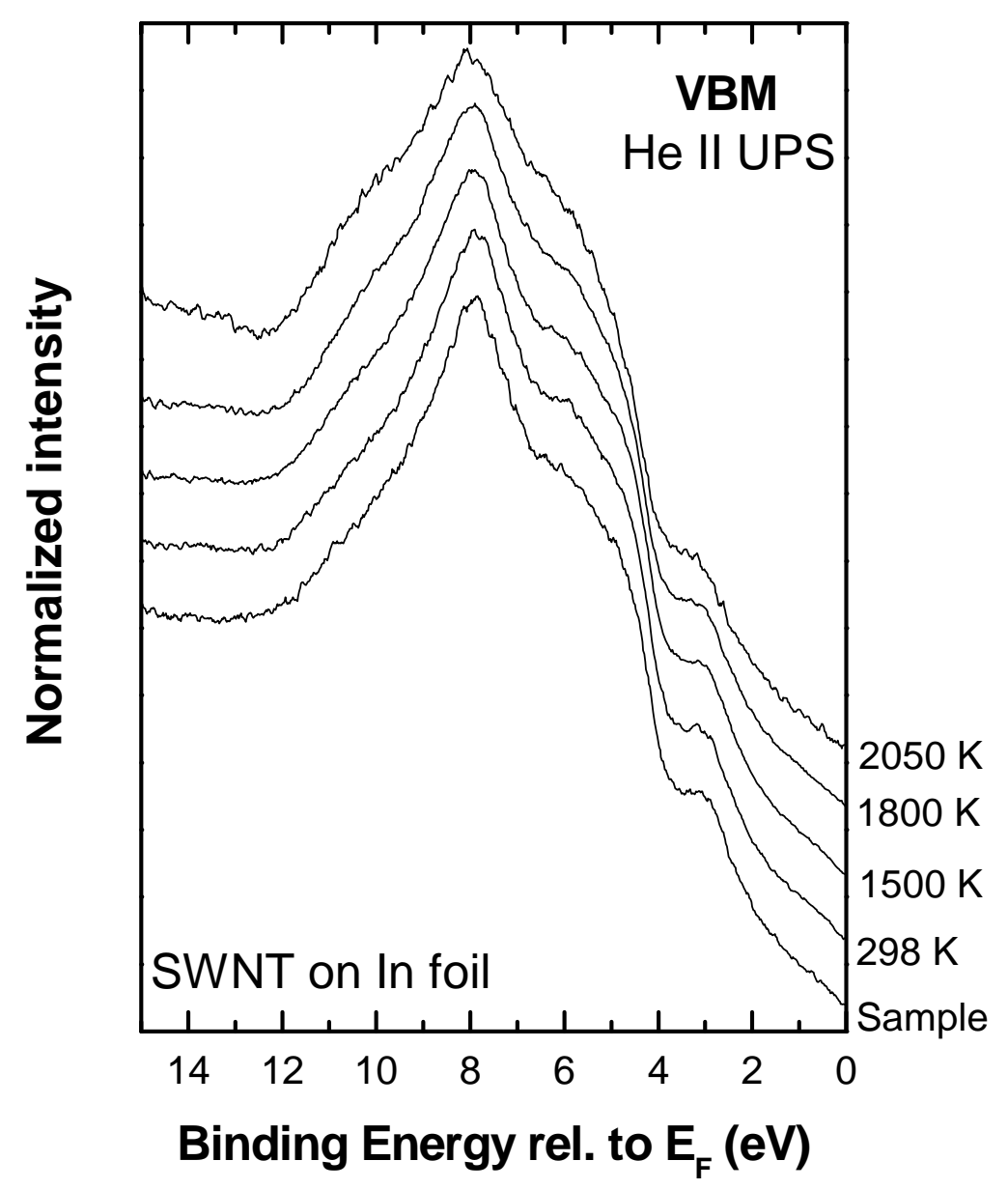

Fig. 26: Valence band structure for SWNTs hydrogenated at different source temperatures. 
Fig. 27 exemplarily shows one of many data sets in which we monitor the oxidation behavior of Ti atoms adsorbed on SWCNTs (in this case a dense film on a Ta foil substrates). As is evident from the spectra, the adsorbed $\mathrm{Ti}$ atoms change from $\mathrm{Ti}$ metal to $\mathrm{Ti}$ oxide species ( $\mathrm{TiO}$ and $\mathrm{TiO}_{2}$ ) as a function of time (on the time scale of a few minutes), with $\mathrm{TiO}_{2}$ being the more stable phase. Various schemes to slow down this oxidation were tested, including the co-deposition of $\mathrm{Li}$, as will be discussed below. In Fig. 16, a C 1s XPS spectrum of a pristine layer of SWCNTs (black curve) is compared with the same film after Ti deposition (red). The observed shift and lineshape variation clearly indicates a charge transfer between the adsorbed $\mathrm{Ti}$ atoms and the $\mathrm{C}$ atoms of the SWCNT.

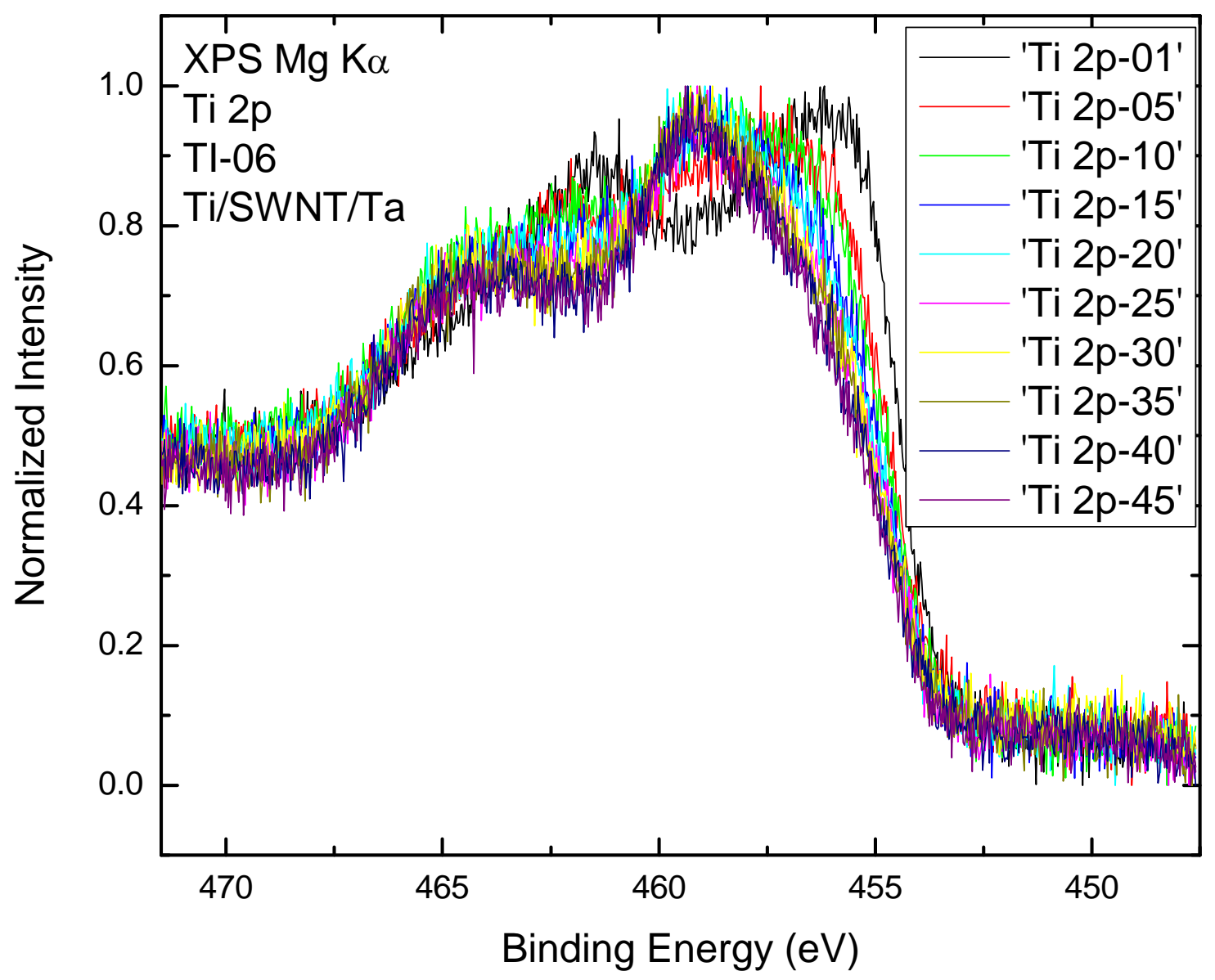

Fig. 27: Time-dependent monitoring of Ti 2p XPS peaks for Ti adsorbed on a dense film of SWCNTs on Ta foil. The inset gives the time (in minutes) of "exposure" to ultra-high vacuum after the first spectrum. 
To study the impact of $\mathrm{Li}$ co-deposition on the oxidation behavior of $\mathrm{Ti}$ adatoms, we have studied the morphology and electronic structure of a Au surface after $\mathrm{Li}$ deposition $(\mathrm{Li} / \mathrm{Au})$. This system is compared to the Au surface after Ti deposition (Ti/Au) and used as a reference for investigating the Li behavior on carbon-based nanomaterials and its impact on Hydrogen storage applications.

A layer of Au was deposited on a Si wafer and exposed to a Li dispenser element getter source for 2 minutes. The deposited $\mathrm{Li}$ forms a continuous layer on the Au surface. The thickness of the layer is about $2.8 \mathrm{~nm}$, calculated by attenuation of Au $4 \mathrm{f} x$-ray photoelectron spectroscopy (XPS) signal after Li deposition. It was shown earlier that Ti deposited at similar conditions forms clusters. This indicates that $\mathrm{Li}$ reacts with the substrate much more strongly than $\mathrm{Ti}$.

Fig. 28 shows O 1s XPS spectra of the sample before and after Li deposition. It is clear that Li deposition induces a strong feature around $530 \mathrm{eV}$, which is interpreted as a metal oxide. A similar change was previously observed on the Ti/Au sample. However, in the latter case, the induced feature had much smaller spectral intensity, indicating that Li reacts with $\mathrm{O}$ more aggressively than $\mathrm{Ti}$. This, in turn, suggests that $\mathrm{Li}$ can be used to "liberate" $\mathrm{Ti}$ atoms from oxygen bonds, making it available (again) for hydrogen bonding. The different behaviors of $\mathrm{Li} / \mathrm{Au}$ and $\mathrm{Ti} / \mathrm{Au}$ thus provide valuable information for future studies of $\mathrm{Li}$-decorated carbonbased nanomaterials.

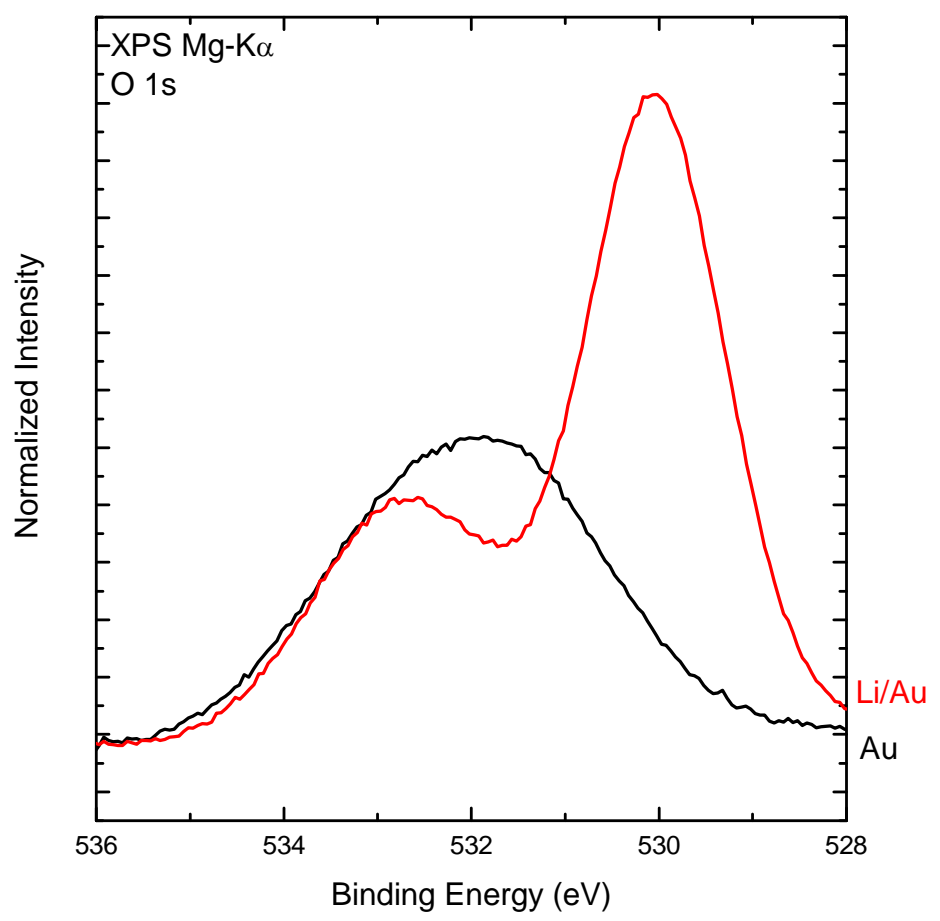

Fig. 28: O 1s XPS spectra of Au sample before and after Li deposition. 
The evolution of the XPS C 1s spectra of a single-walled carbon nanotubes (SWNTs) sample after $30 \mathrm{sec}$ (nominal thickness $1 \AA$ ) and 2 min Ti deposition is shown in Fig. 29a and Fig. 29b, respectively. In both cases, a small $(\sim 0.1 \mathrm{eV})$ but significant chemical shift toward higher binding energy (BE) was observed, indicating a change of the chemical environment for carbon atoms by the presence of $\mathrm{Ti}$ adatoms. Furthermore, the main $\mathrm{C} 1$ s peak broadens at the high $\mathrm{BE}$ side, which is attributed to a "disturbed" carbon network by Ti adatoms. Fitting $C 1$ s spectra (not shown here) provides a quantification for the $\mathrm{sp}^{2}$ and $\mathrm{sp}^{3}$ components of the main $\mathrm{C} 1 \mathrm{~s}$ peak, which shows an increase of the relative $\mathrm{sp}^{3} / \mathrm{sp}^{2}$ ratio after Ti deposition. The most distinguishable feature in Fig. 29 is the emergence of a new peak for the 2 min Ti-deposited sample at around $282.5 \mathrm{eV}$, which is ascribed to Ti-C bond formation. The ratio of the Ti-C component to the $\mathrm{C} 1 \mathrm{~s}$ peak is $\sim 1 \%$, and apparently depends on the amount and coverage of the deposited Ti. For a similar 2 min deposition of Ti on a $\mathrm{C}_{60}$ sample (nominal thickness $4 \AA$ ), shown in Fig. 30, a charge transfer through hybridization of the Ti $3 \mathrm{~d}$ orbital and the HOMO and LUMO levels of the $\mathrm{C}_{60}$ molecules leads to a chemical shift of $\sim 0.3 \mathrm{eV}$ (after correction for sample charging effects) towards lower $\mathrm{BE}$ of the $\mathrm{C}$ 1s peak. The characteristic shake-up satellite features at the high BE shoulder of the main C 1s peak (Fig. 23b) are preserved after Ti deposition, indicating no disruption of the cage-like $\mathrm{C}_{60}$ molecules upon deposition of Ti adatoms.

We have shown that the deposited Ti adatoms on carbon nanostructures oxidize even under UHV conditions. However, no change of the $C 1$ s spectra was observed during the oxidation process of the Ti overlayer, which indicates that the chemical structures at the Ti-carbon interface are not affected by the adsorption of oxygen species and subsequent oxidation of the outer metal layer.
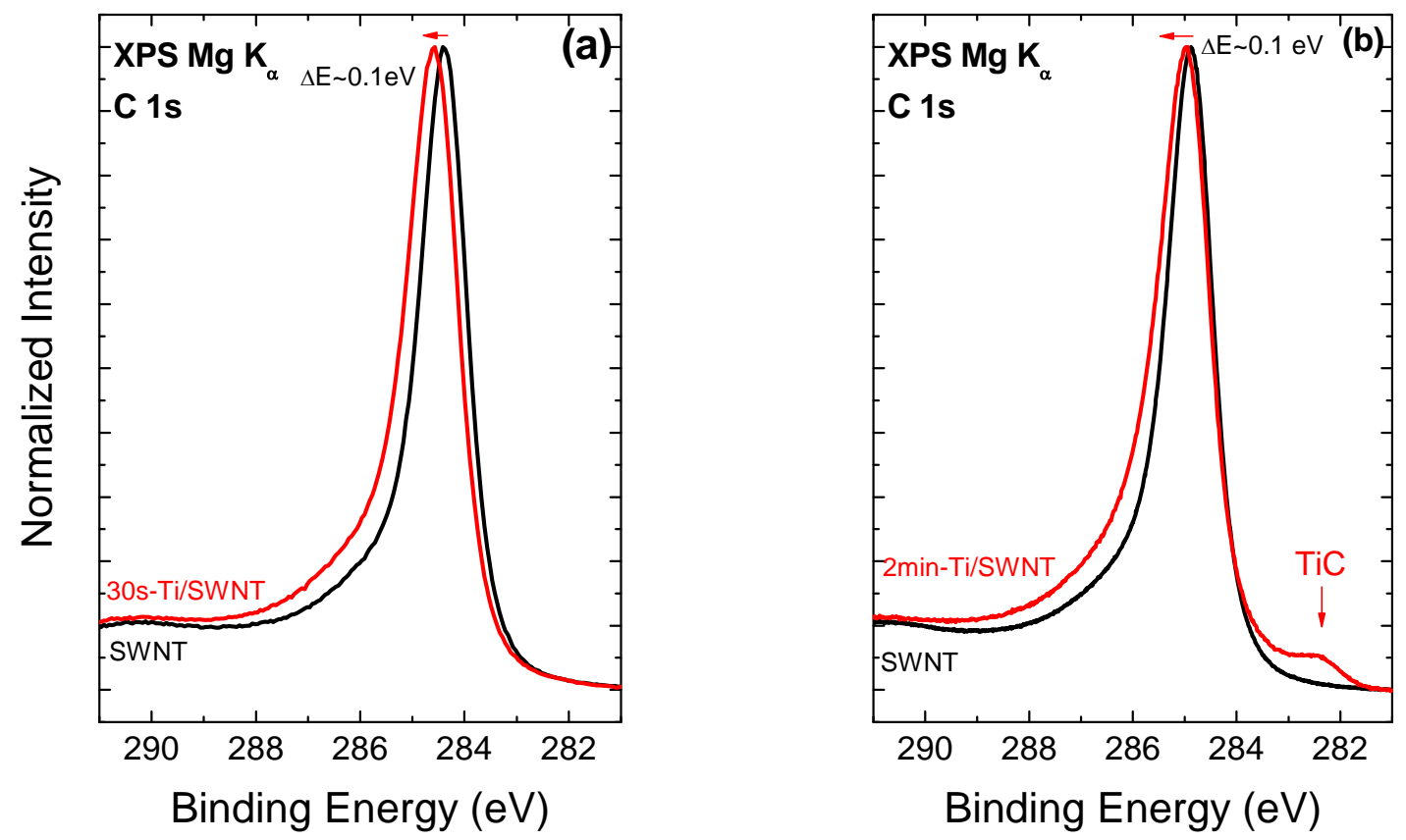

Fig. 29: Evolution of C 1s XPS spectra of SWNT sample after 30 sec (a) and 2 min (b) Ti deposition. 

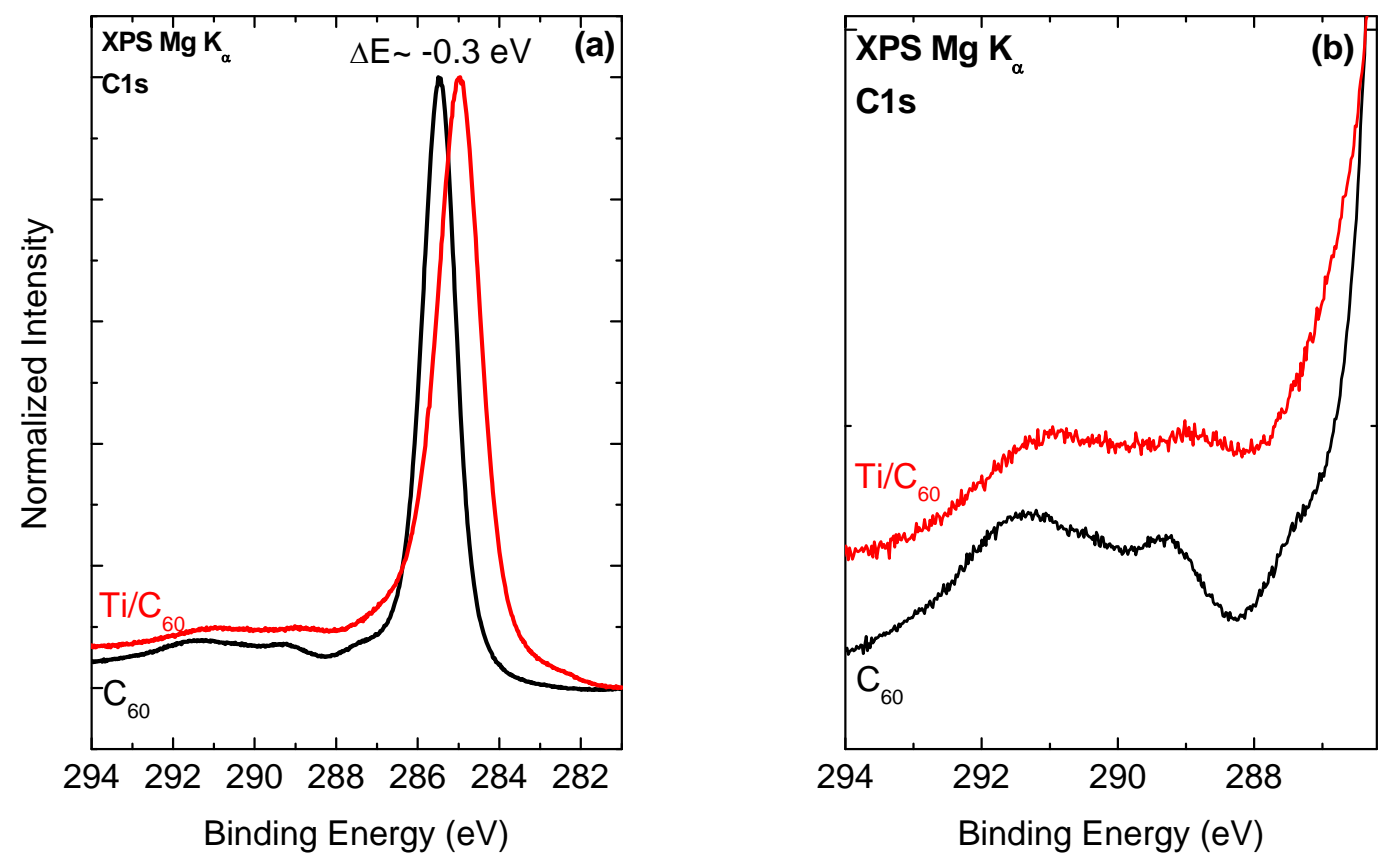

Fig. 30: Spectral changes of the C 1s core level due to 2 min Ti deposition on a $\mathrm{C}_{60}$ sample; (a) the main peak shifts $0.3 \mathrm{eV}$ to lower BE; (b) Shake-up satellites at the high BE shoulder of the main peak.

Among the achievements of this project, we were particularly honored to have a report on our research efforts accepted for publication in the Journal of the American Chemical Society (26). The paper describes qualitatively the oxidation behavior of Ti-decorated SWNTs in ultra-high vacuum (UHV) condition, which could be an obstacle for their hydrogen storage capability, and our proposal for an approach to overcome this problem by co-evaporation of Li. To understand the complicated oxidation behavior of Ti adatoms on SWNTs, we have developed an analysis approach to decompose multi-component XPS spectra of $\mathrm{Ti}$ on SWNTs. The approach is important to identify (and later quantify) various intermediate states of Ti oxides, which are not easy to define for fast dynamical changes of Ti adatoms (even under UHV conditions). More importantly, the approach helps to unravel experimentally the signature of the Ti-C bonds between $\mathrm{Ti}$ adatoms and the $\mathrm{C}$ atom network of SWNTs, which, so far, have only appeared in theoretical works as hypotheses with regards to metal-carbon interactions, especially in the decoration regime.

We have also applied the developed methodology to experimental data obtained from the investigation of time-dependent oxidation processes of Ti-decorated SWNTs under UHV, and their subsequent reduction by $\mathrm{Li}$. The goal is to quantify the oxidation and reduction behavior of $\mathrm{Ti}$ adatoms on SWNTs and to investigate the effects of this oxidation and reduction on the presence of Ti-C bonds. This would help to understand the mechanisms and dynamics of the Ti- 

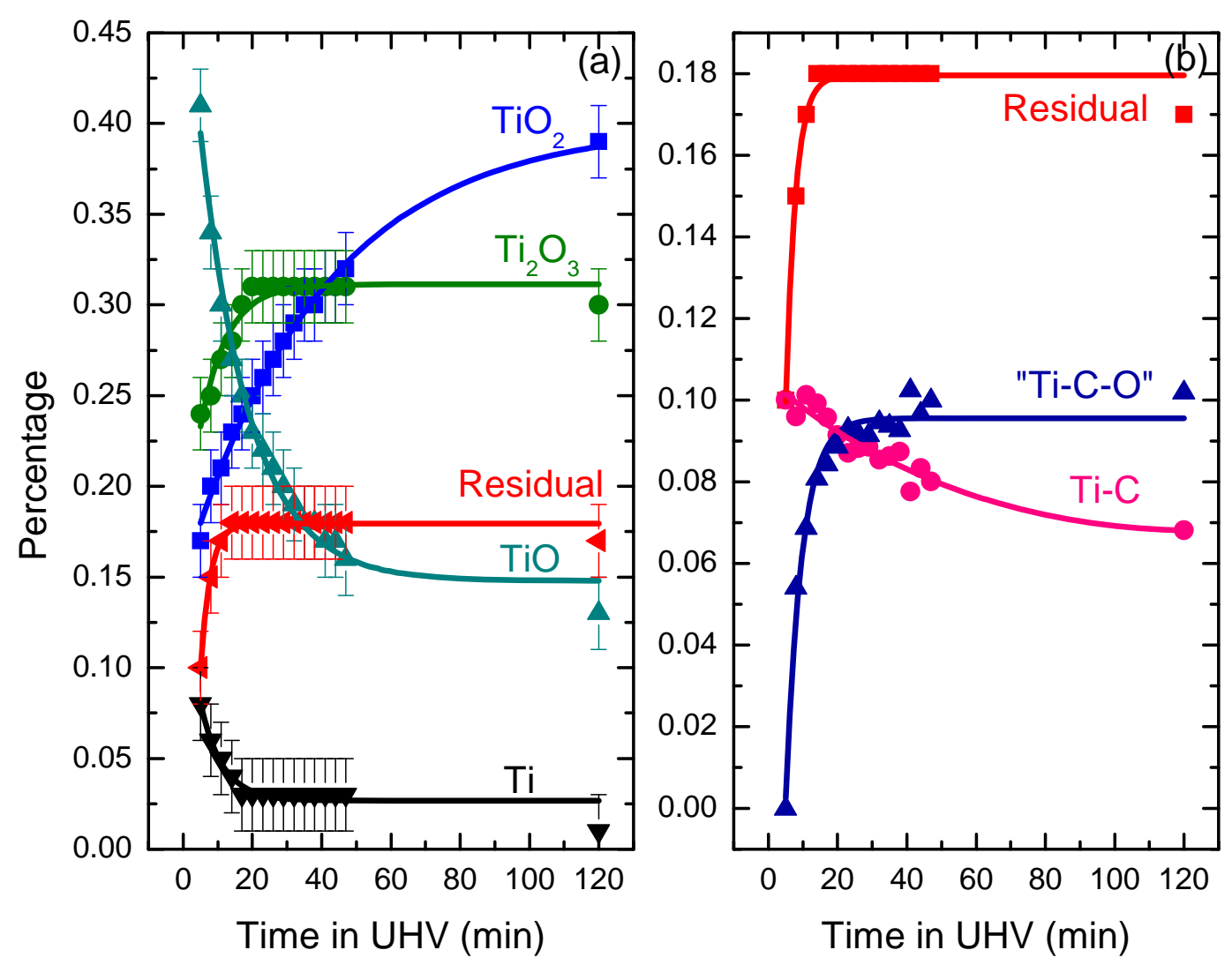

Figure 31: Evolution of Ti-derived components (Ti oxides and Ti-C species) for a Ti/SWNT sample as a function of time in UHV. See text for the identification and description of the Ticomponents.

C interactions with the surroundings (e.g. oxidation attacks, interactions with a third partner such as $\mathrm{Li}$ or $\mathrm{H}_{2}$ ).

Fig. 31a shows the evolution of the Ti oxide components after deposition on SWNTs as a function of time in UHV. The Ti oxide components were identified in agreement with available literature data and their integrated areas were derived from the decomposition of the $\mathrm{Ti} 2 \mathrm{p}$ envelop spectra. It is evident that the Ti adatoms, deposited as metallic Ti in UHV, oxidize and transform to Ti oxides. We observe dramatic changes of the Ti adatom spectra in the first 20-25 min after deposition on SWNTs, which is attributed to the interactions of Ti adatoms with oxygen adsorbed on the surface of SWNTs prior to the deposition (rather than adsorption of oxygen from residual gas in vacuum by $\mathrm{Ti}$ adatoms after deposition). This argument is also supported later by the stoichiometry analysis in conjunction with Fig. 32. Once again, the spectral decomposition approach allows a full evaluation of the evolution of the $\mathrm{Ti}$ oxide components. The $\mathrm{TiO}$ component decreases, while the $\mathrm{TiO}_{2}$ component increases and a stabilization of $\mathrm{Ti}_{2} \mathrm{O}_{3}$ component is observed after an initial increase. The results indicate 


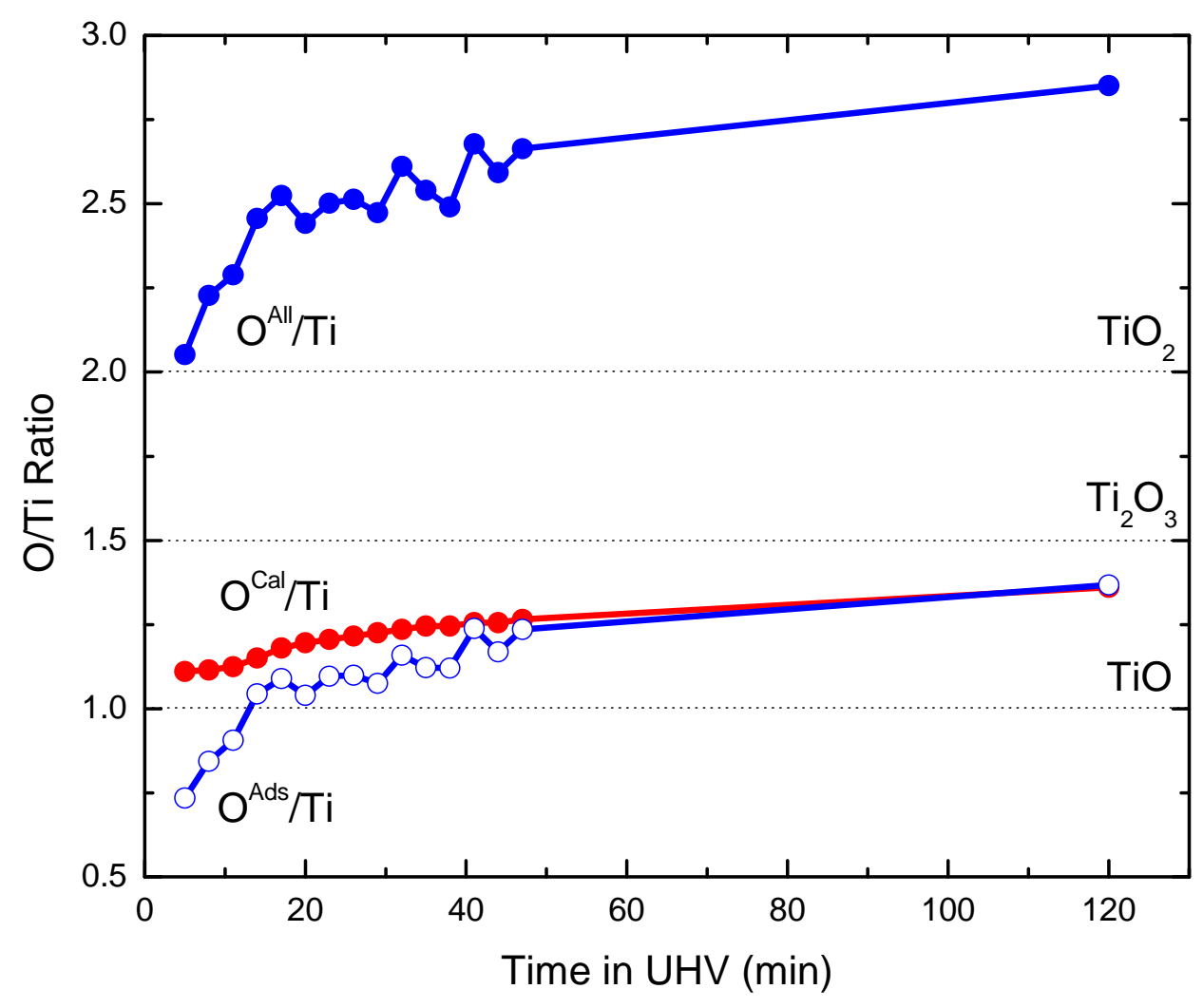

Figure 32: Evolution of the stoichiometry of Ti oxides for a Ti/SWNTs sample as a function of time in UHV. The $\mathrm{O}^{\mathrm{Cal}} / \mathrm{Ti}$ ratio reflects the calculated stoichiometry of Ti oxide components from Fig. 1.

dynamic changes of $\mathrm{Ti}$ oxides from lower, intermediate and unstable to higher, stable oxidation states.

The residual derived from the above-mentioned decomposition of the Ti oxides (the evolution is shown by the red line in Fig. 31a) was further fitted with two additional features (not shown). This resulted in Ti 2p $\mathrm{p}_{3 / 2}$ peaks at 454.7 and $456.3 \mathrm{eV}$. Comparing with literature data, the peak at $454.7 \mathrm{eV}$ is assigned to a Ti-C component and the peak at $456.3 \mathrm{eV}$ is ascribed to "Ti-C-O". The evolution of the Ti-C and "Ti-C-O" components is shown in Fig. 1b. We observe a decrease of the Ti-C component with time in UHV, contrary to an increase of the "Ti-C-O" component, suggesting a partial oxidation of $\mathrm{Ti}$ atoms in $\mathrm{Ti}-\mathrm{C}$ bonds and the transformation of $\mathrm{Ti}-\mathrm{C}$ to ternary Ti-C-O compounds. The results directly provide details about the interaction of $\mathrm{Ti}$ adatoms with $\mathrm{C}$ atoms of SWNTs via the formation of Ti-C bonds, as well as their oxidation behaviors. Again, thermodynamical analyses, probably with reference to theoretical work, might be required to fully understand the mechanisms of the Ti-C bond formation and oxidation. 
The identification and quantification of the Ti oxide components provide a practical way to evaluate the actual "average stoichiometry" of the Ti oxides formed on the surface of SWNTs. Fig. 32 shows the evolution of the $\mathrm{O} / \mathrm{Ti}$ ratios as a function of time in UHV obtained from the experiments (blue lines) as well as calculated from chemical compositions of the decomposed $\mathrm{Ti}$ oxide components (red line). The experimental $\mathrm{O} / \mathrm{Ti}$ ratios were estimated from the integrated areas under the XPS O 1s and Ti 2p peaks, respectively, after subtraction of a linear background and taking the corresponding photoionization cross-sections into account. The solid blue line $\left(\mathrm{O}^{\mathrm{All}} / \mathrm{Ti}\right)$ was derived for the total oxygen signal from the Ti-deposited sample, while the circle blue line $\left(\mathrm{O}^{\mathrm{Ads}} / \mathrm{Ti}\right)$ represents only oxygen atoms adsorbed to Ti-decorated SWNTs after deposition (i.e., without the contribution of oxygen previously adsorbed on the surface of SWNTs). We argue that the incoming metallic Ti adatoms, while covering the SWNTs surface (and interact with $\mathrm{C}$ atoms), would interact with (and "consume") oxygen already adsorbed on the SWNTs. Thus, the $\mathrm{O}^{\mathrm{All}} / \mathrm{Ti}$ ratio would over-estimate, while the $\mathrm{O}^{\mathrm{Ads}} / \mathrm{Ti}$ ratio would underestimate the contribution of oxygen to the formation of Ti oxides. As seen in Fig. 32, by detail quantification of the Ti oxide components, we can observe the following. First, we can confirm our hypothesis about the contribution of oxygen previously adsorbed on the surface of SWNTs to the oxidation of Ti adatoms. Secondly, a clear two-step process of the oxidation can be observed by comparing $\mathrm{O}^{\mathrm{Cal}} / \mathrm{Ti}$ to $\mathrm{O}^{\mathrm{Ads}} / \mathrm{Ti}$. In the first 20-25 min after Ti deposition, the large deviation of $\mathrm{O}^{\mathrm{Ads}} / \mathrm{Ti}$ from $\mathrm{O}^{\mathrm{Cal}} / \mathrm{Ti}$ indicates an under-estimated contribution of oxygen on SWNTs, which in turn suggests a $\mathrm{O}^{\mathrm{SWNT}}$-dominated oxidation of Ti adatoms (i.e., the oxidation is mostly driven by the interaction of the Ti adatoms with oxygen already on the surface of SWNTs $\left(\mathrm{O}^{\mathrm{SWNT}}\right)$, rather from the residual gas, $\mathrm{O}^{\mathrm{Ads}}$ ). After the depletion (by "consumption" of $\mathrm{Ti}$ ) of the $\mathrm{O}^{\mathrm{SWNT}}$, the convergence between $\mathrm{O}^{\mathrm{Cal}} / \mathrm{Ti}$ and $\mathrm{O}^{\mathrm{Ads}} / \mathrm{Ti}$ suggests that adsorption of oxygen from the residual gas dominates the oxidation of $\mathrm{Ti}$ in this stage, which supplies oxygen for the transformation of Ti from lower to higher oxidation states.

We have earlier discussed the effects of $\mathrm{Li}$ in the reduction of $\mathrm{Ti}$ oxide $\left(\mathrm{TiO}_{2}\right)$ on $\mathrm{Au}$ and on SWNTs. The observed Li-induced shift of spectral weight of Ti 2p spectra to lower binding energy is explained by the reduction of $\mathrm{TiO}_{2}$ and reformation of Ti oxides in lower oxidation states $\left(\mathrm{Ti}_{2} \mathrm{O}_{3}\right.$ and $\left.\mathrm{TiO}\right)$. We have also argued that the Ti-C bonds would be re-emerging during this reduction process. However, direct attempts to quantify these reduction effects of Ti oxides by Li using the above-mentioned decomposition of XPS data were hampered by the fact that we observe a systematic shift of all core-level spectra to higher binding energy after Li deposition (C 1 s shifts by $0.3 \mathrm{eV}$ and $\mathrm{O} 1 \mathrm{~s}$ shifts by $0.7 \mathrm{eV}$ after $\mathrm{Li}$ deposition on $\mathrm{TiO}_{2} / \mathrm{SWNT}$ ). The Liinduced shifts are attributed to charge transfer from $\mathrm{Li}$ to underlying species, which produces a band bending at the surface, and consequently, a shift to higher binding energy of the core levels, including Ti $2 p$ spectra (For $\mathrm{O} 1 \mathrm{~s}$, the shift is more complex due to the involvement of the oxidation process). By comparing our experimental data with literature data on the BE shifts, induced by $\mathrm{Li}$ on $\mathrm{TiO}_{2}$ and $\mathrm{Li}$ on SWNT, (and also from our decomposition using stepwise corrections for the Li-induced shift), we determined a shift of $0.3 \mathrm{eV}$ for the Ti $2 \mathrm{p}$ spectra of our $\mathrm{Li} / \mathrm{TiO}_{2} / \mathrm{SWNT}$ sample. A Li-induced-0.3eV-shift-corrected decomposition of the $\mathrm{Ti} 2 \mathrm{p}$ spectrum for a $\mathrm{Li} / \mathrm{TiO}_{2} / \mathrm{SWNT}$ sample after $5 \mathrm{~min}$ of $\mathrm{Li}$ deposition is shown in Fig. 33a. The reduction of Ti oxides to lower oxidation states is confirmed by the contributions of $\mathrm{Ti}_{2} \mathrm{O}_{3}$ and TiO components. Furthermore, the residual was also fitted, as shown in Fig. 33b, with two features similar to the ones applied to the decompositions of the Ti/SWNTs series: one at 455.3 $\mathrm{eV}$ for the Ti-C bonds and one at $456.5 \mathrm{eV}$ for a possible "Li-Ti-C-O" compound. The results 
confirm our suggestion that oxidized Ti-C bonds on SWNT sample, indeed, re-emerged after additional deposition of Li. This is important as it proves our concept of using a co-deposition with Li to counteract the oxidation of Ti on SWNTs, which would open up the possibility of using metal-decorated SWNTs as a candidate material for hydrogen storage.

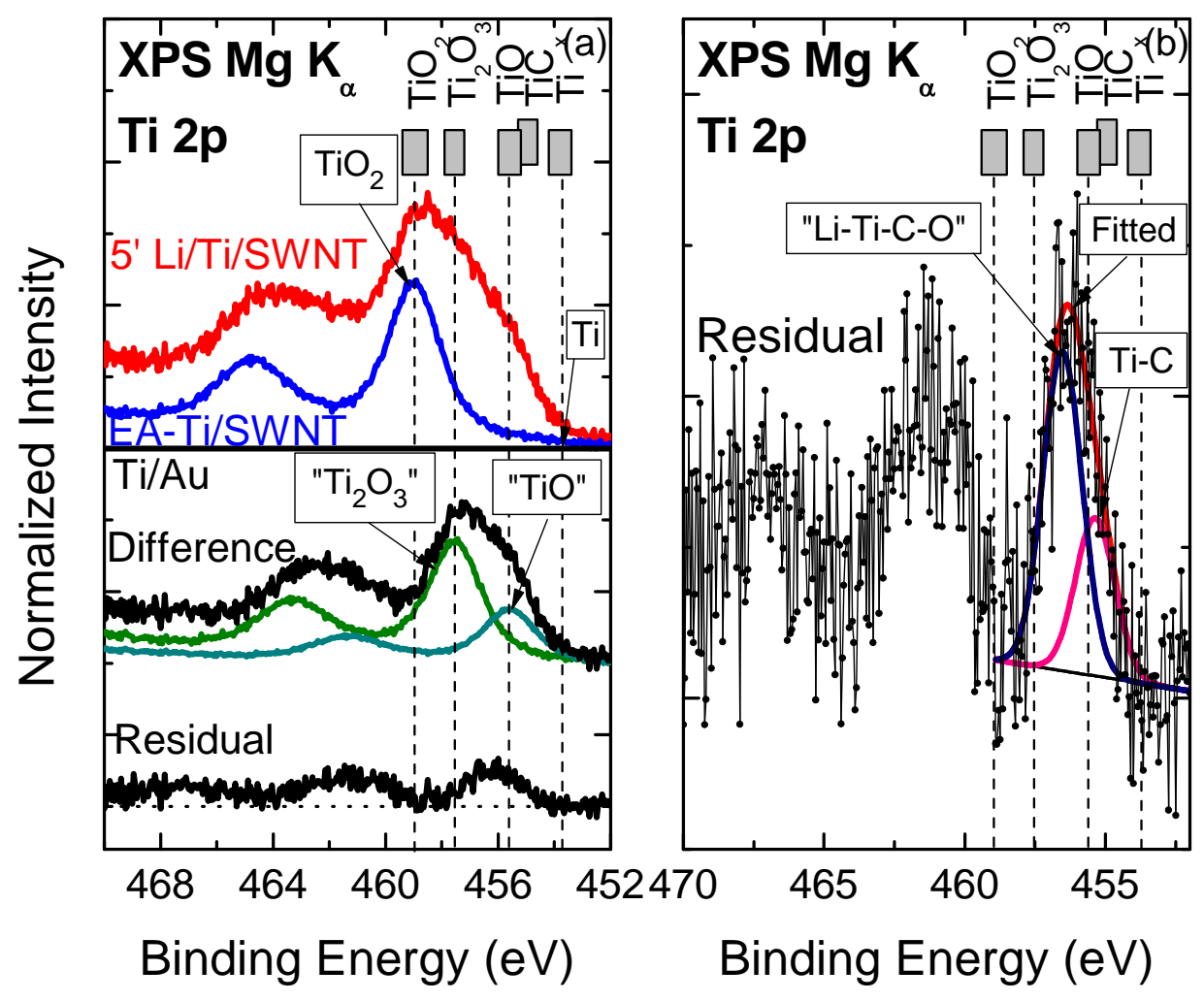

Figure 33: (a) Decomposition of a Ti 2p spectrum of the Li/Ti/SWNT sample 5 min after Li deposition. The spectrum was shifted by $-0.3 \mathrm{eV}$ to account for a Li-induced shift of all electronic levels. (b) The residual was further fitted with two features to show the re-emergence of Ti-C bonds and the contribution of a Li-Ti-C-O compound upon Li deposition. 


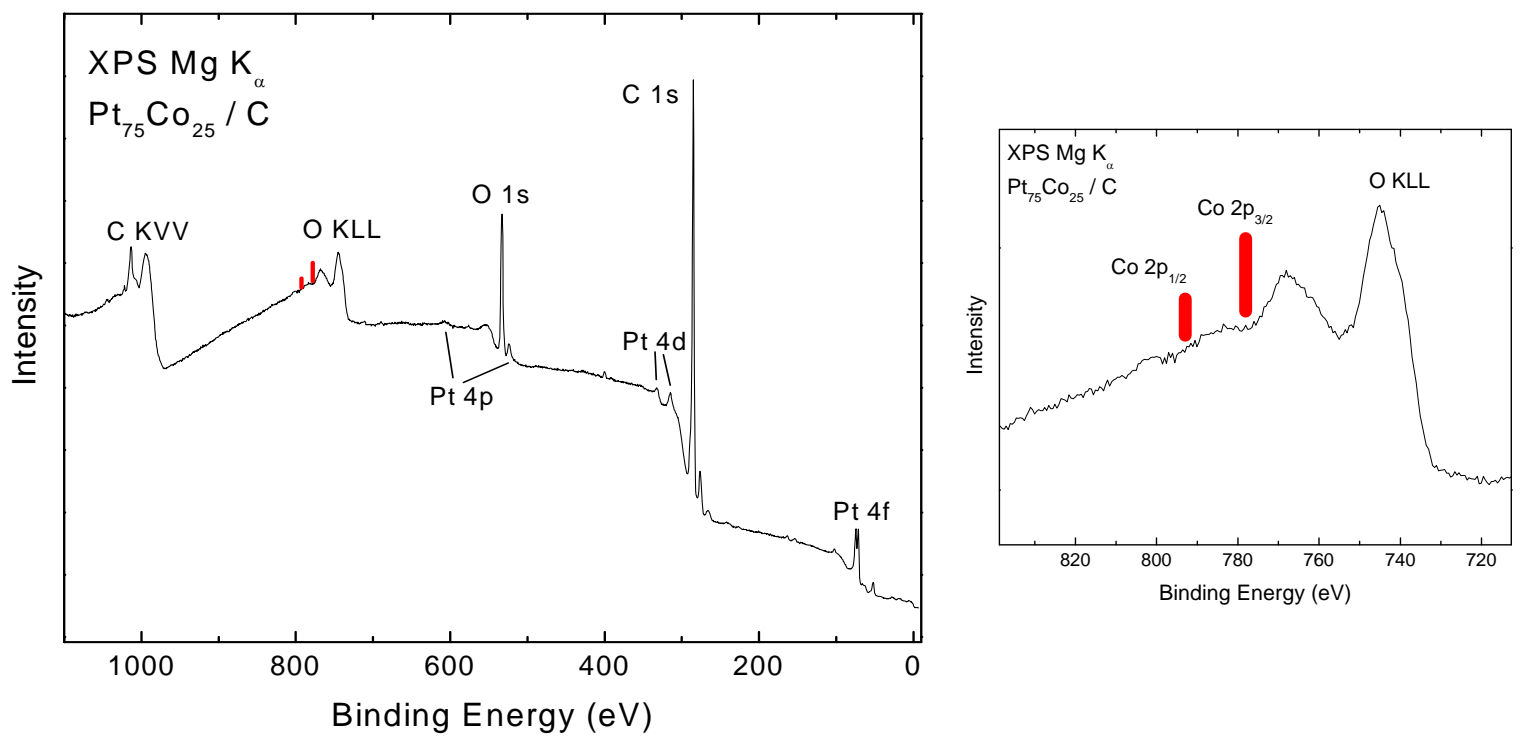

Fig. 34: XPS spectra of a $\mathrm{Pt}_{75} \mathrm{Co}_{25} / \mathrm{C}$ powder sample. The red lines indicate position and intensity of the Co 2p lines if the Pt:Co surface stoichiometry would be 3:1.

\section{Results of Task 5/Task 2.1:}

In close collaboration with United Technologies Corporation (UTC), this task focused on a detailed analysis of the electronic and chemical surface properties of nanomaterial fuel cell catalysts from UTC. We have measured powder samples of Pt and Co containing catalyst and reference materials for fuel cell applications (from UTC) with photoemission (at UNLV) as well as with $\mathrm{x}$-ray absorption and x-ray emission (at the Advanced Light Source in Berkeley).

The catalyst samples consisted of a Pt/Co alloy on a C support. However, our surface-sensitive XPS experiments (information depth $\sim 1-3 \mathrm{~nm}$ ) only show the lines of Pt, C, and O, but no Co, as is exemplarily shown for $\mathrm{Pt}_{75} \mathrm{Co}_{25} / \mathrm{C}$ in Fig. 34. In contrast, we can find $\mathrm{Co} \mathrm{x}$-ray emission on those samples. X-ray emission is a photon-in photon-out technique and hence is more bulk sensitive (information depth of typically $>100 \mathrm{~nm}$ ). This suggests that the Co atoms in the investigated samples are buried below a layer of Pt and/or C.

Furthermore, our spectra reveal oxidation of Pt on some of the samples, whereas Co is not oxidized at all. This information about oxidation of the catalyst material is one of the key questions for stability issues of the fuel cell. 


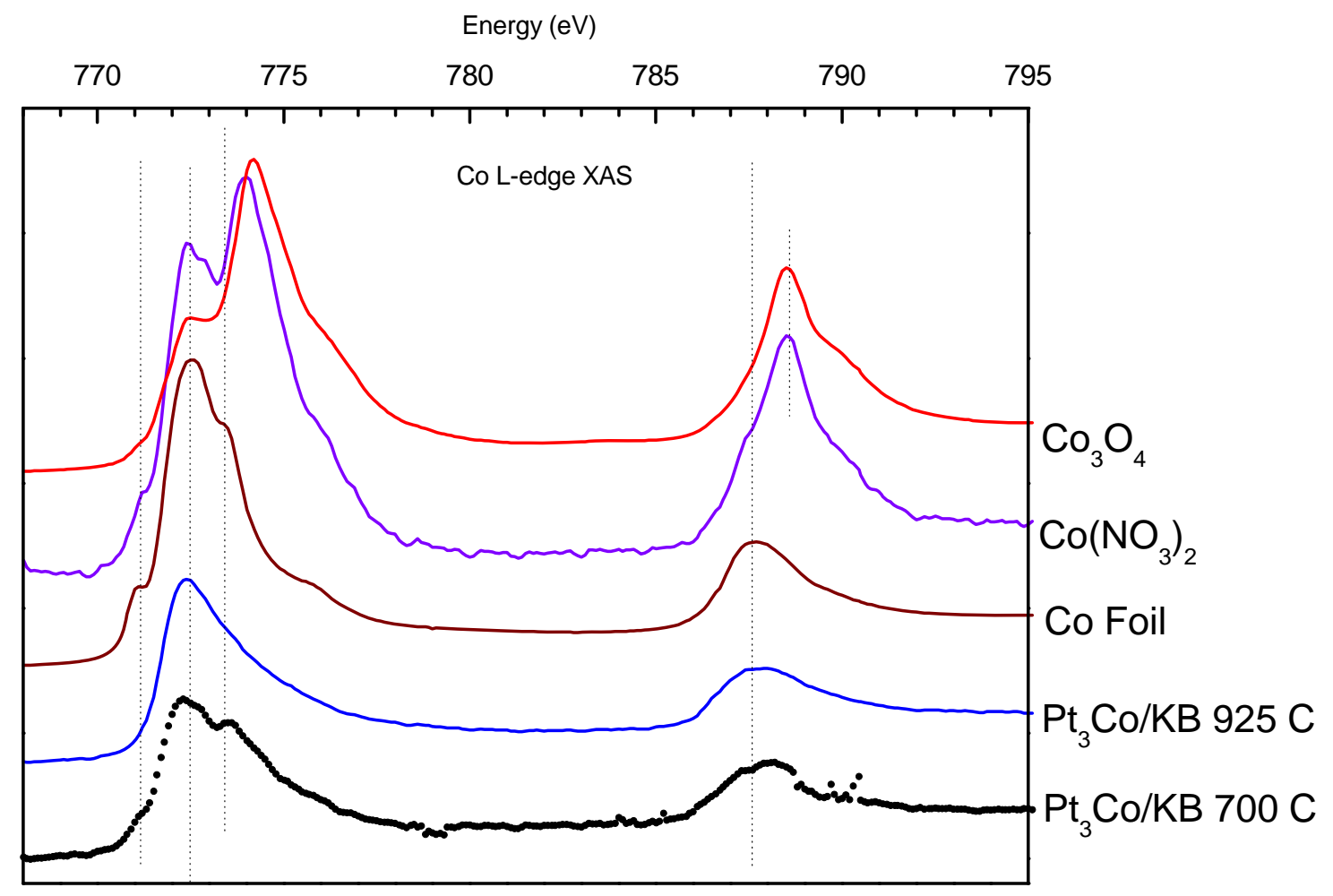

Fig. 35: Co L-edge XAS from $\mathrm{Pt}_{3} \mathrm{Co}$ catalysts, annealed at different temperatures, and reference samples.

To further understand the chemical properties, investigate the presence (or absence) of a coreshell structure, and gain insight into the electronic properties, we have conducted synchrotronbased soft $\mathrm{x}$-ray spectroscopies at the ALS. Fig. 35 shows the Co L-edge X-ray absorption spectroscopy (XAS) spectra from reference samples and catalysts treated at different temperatures. As can be seen from the figure, the Co L-edge spectra vary with treatment temperature. By comparing to reference samples, Co in the catalysts transforms gradually from the Co-containing precursor into the metallic Co form. As expected, the influence of oxygen is more pronounced in samples that were treated at lower annealing temperatures.

Co L-edge X-ray emission spectroscopy (XES) data from the same set of samples are shown in Fig. 36. The decrease of spectral intensity on the left hand side of the main features (i.e., at lower photon energies) indicates that Co $3 d$ occupied states follow a similar trend as the unoccupied states in the XAS spectra. The intensity ratio between the two main features (intensity at approx. $792 \mathrm{eV}$ divided by intensity at approx. $777 \mathrm{eV}$ ) also gradually decreases with increasing annealing temperature. This is due to a self-absorption effect that can be used to monitor the density of Co near the emitting atom. Note that the observed ratio for the sample annealed at 925 $\mathrm{C}$ is still larger than that found for Co metal. This indicates that other atomic species (e.g., oxygen) are still present in the vicinity of the emitting Co atom. 


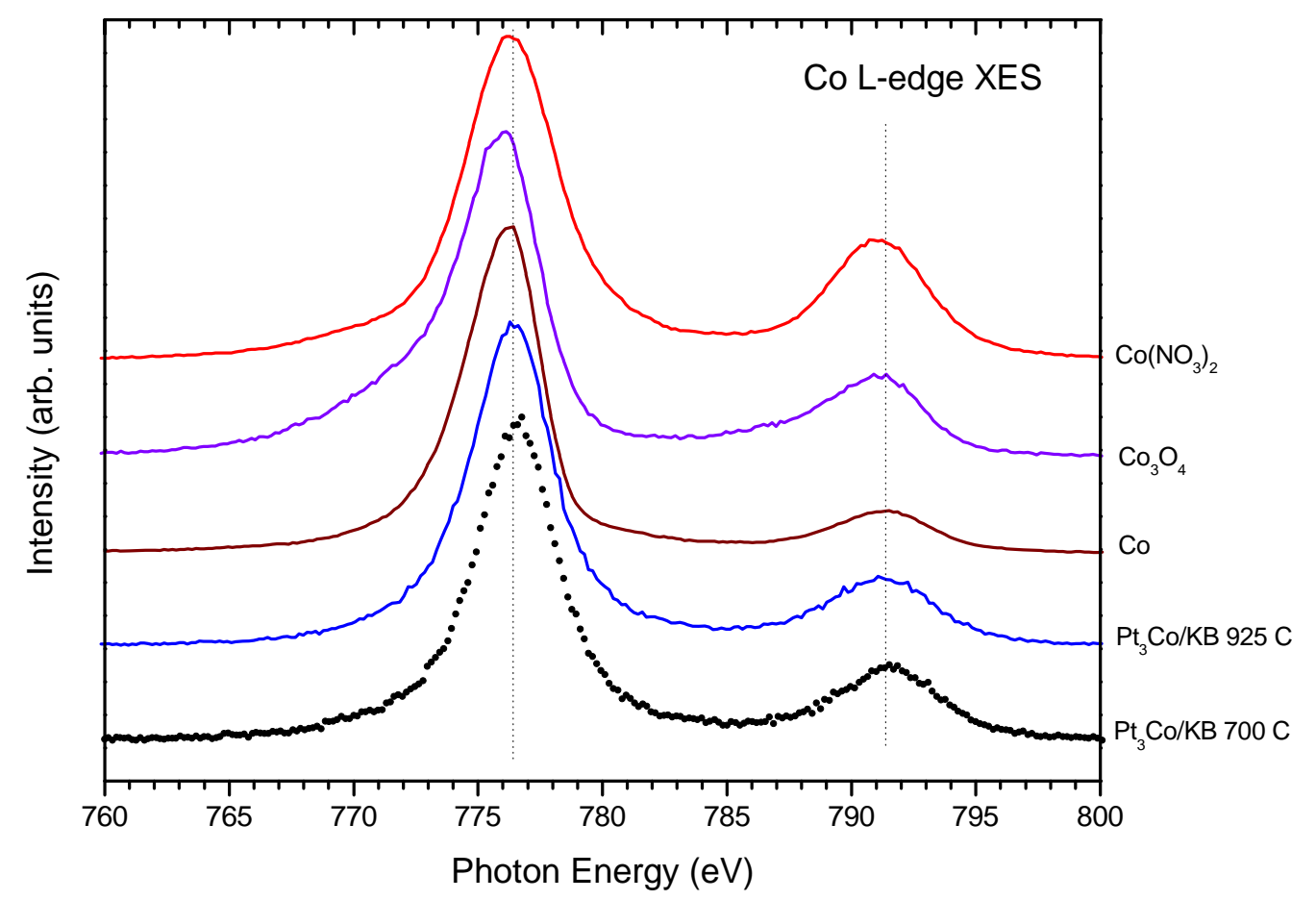

Fig. 36: Co L-edge XES from $\mathrm{Pt}_{3} \mathrm{Co}$ catalysts, annealed at different temperatures, and reference samples.

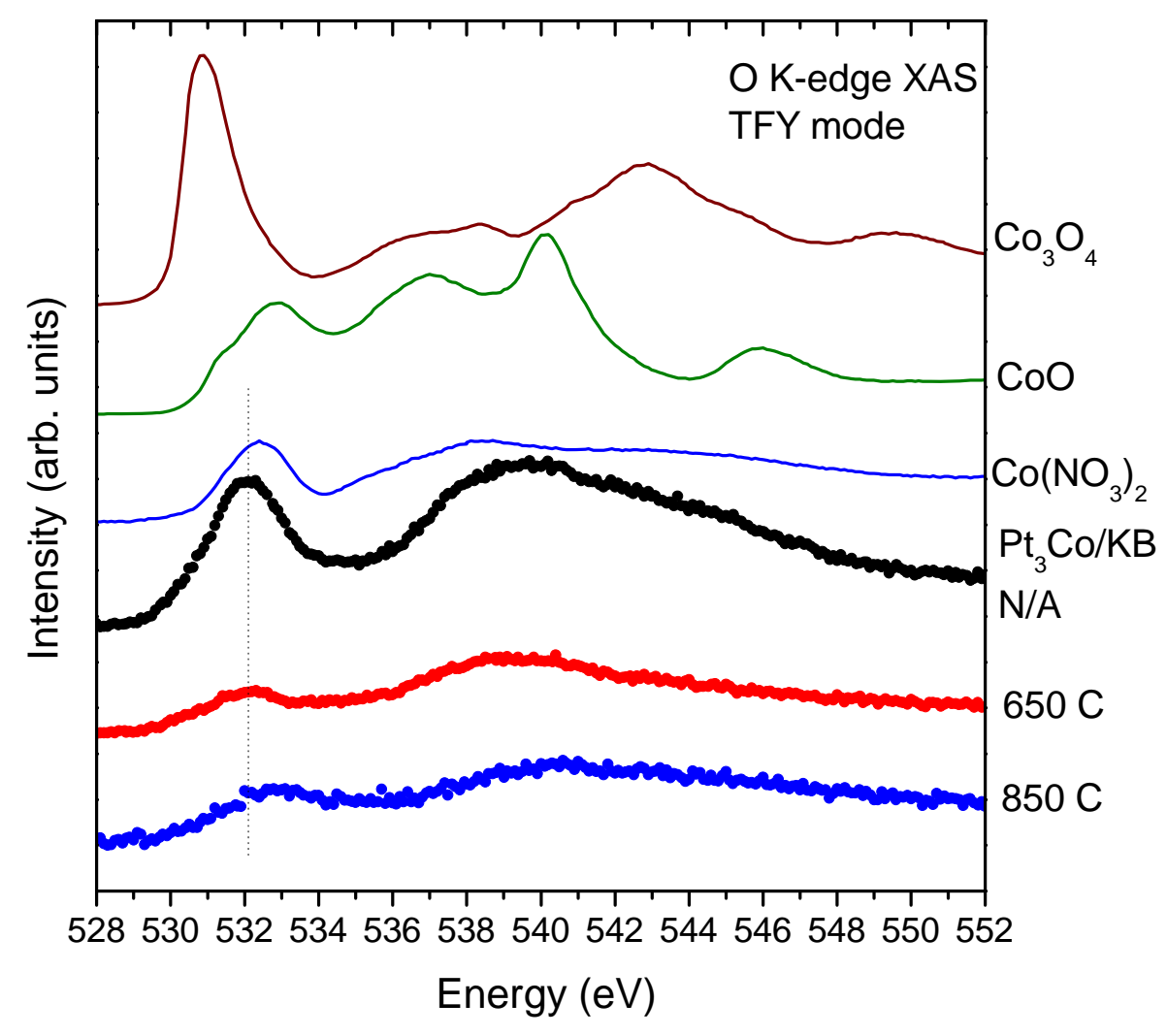

Fig. 37: Comparison of O K-edge XAS spectra between reference samples and catalysts. 


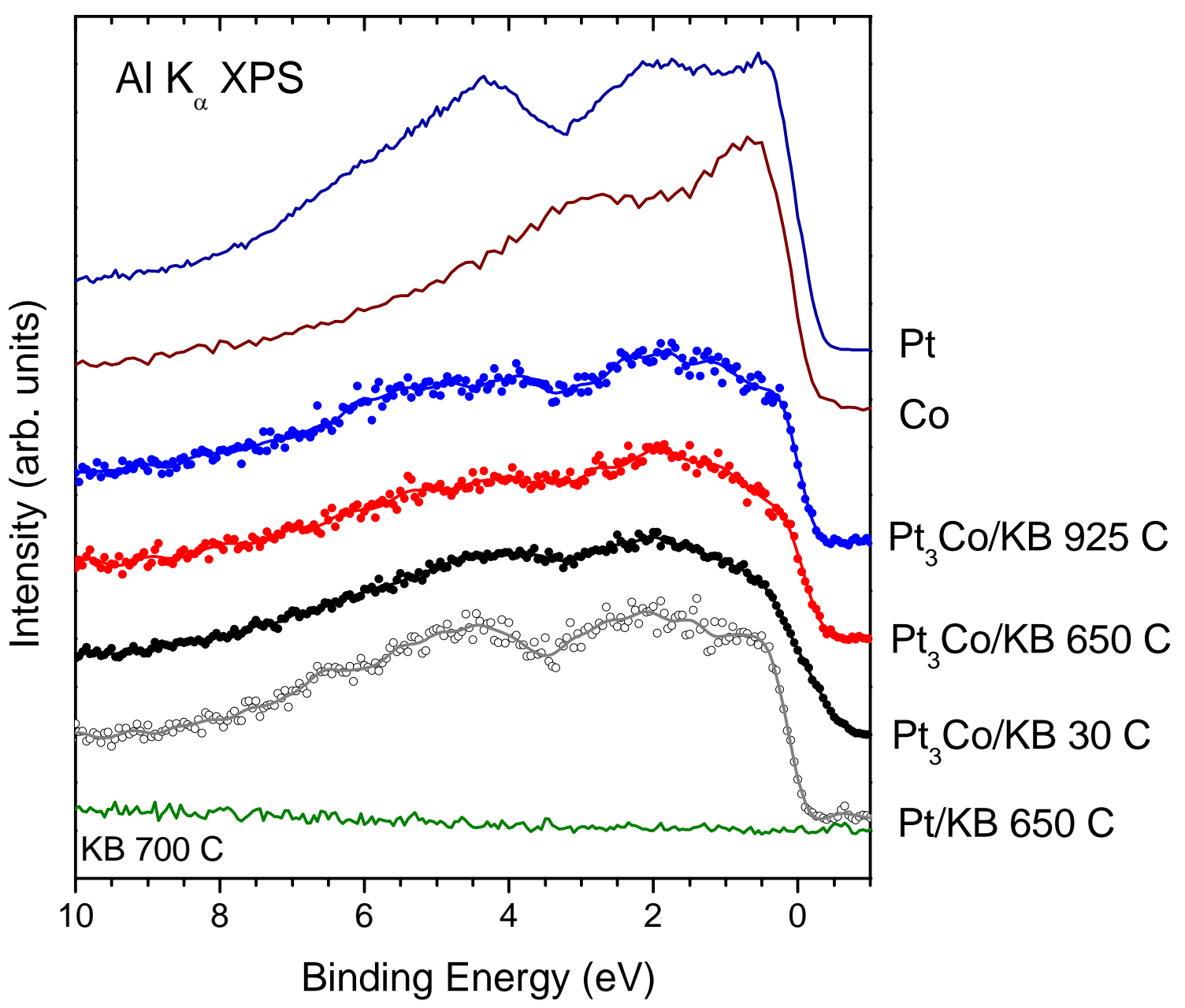

Fig. 38: Valence band structure of reference samples and catalysts.

O K-edge x-ray absorption (XAS) spectra from reference samples and catalysts are shown in Fig. 37. The spectral structure of untreated $\mathrm{Pt}_{3} \mathrm{Co} / \mathrm{KB}$ sample is similar to that of $\mathrm{Co}\left(\mathrm{NO}_{3}\right)_{2}$. The intensity of the resonance at $532.5 \mathrm{eV}$ decreases with increasing annealing temperature. Comparing with XAS of the Co-oxides, we believe that the residual oxygen is still in a nitrite environment (and other oxides), but not in a distinct Co-oxide environment.

Fig. 38 shows valence band structure of reference samples and catalysts treated at different temperatures, recorded with monochromatized $\mathrm{Al} \mathrm{K}_{\alpha}$ XPS. It was found that carbon black (KB) does not contribute any spectral structure to the valence band region (bottom spectrum). The overall valence band of $\mathrm{Pt}_{3} \mathrm{Co} / \mathrm{KB}$ is closer to that of the $\mathrm{Pt}$ foil than of the Co foil, but it is not a simple superposition. The most significant impact of annealing on the valence band structure is observed directly at the Fermi energy - the Fermi edge appears to sharpen with increasing annealing temperature. 


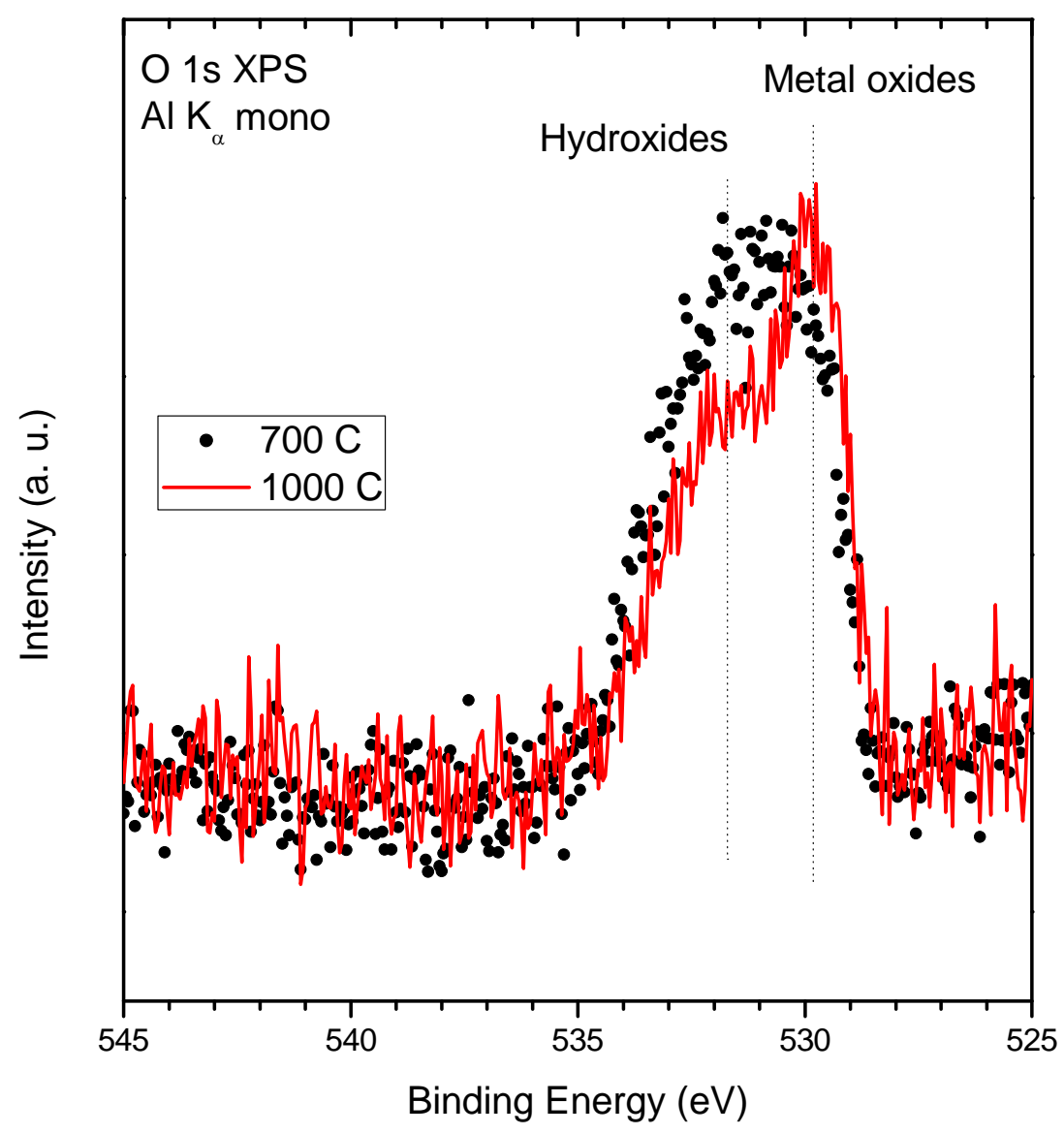

Fig. 39: O 1s XPS of PtIr/KB samples.

O 1s XPS spectra of two PtIr/KB samples are shown in Fig. 39. The main feature consists of at least two components: metal oxides (around $530 \mathrm{eV}$ ) and hydroxides (around $532 \mathrm{eV}$ ). At higher annealing temperature, the spectral intensity of the hydroxide component decreases, and the overall area of the $\mathrm{O} 1 \mathrm{~s}$ feature decreases. This indicates that a higher annealing temperature is more effective in reducing the oxygen content on the nanoparticle catalysts. Meanwhile, the presence of the metal-oxide component in both samples implies that either the current annealing process is not sufficient to fully remove the oxygen content from the samples or that the samples have been re-oxidized after annealing.

Fig. 40 shows Pt and Ir 4f XPS spectra of the samples. At different annealing temperatures, Pt and Ir $4 \mathrm{f}$ features have similar spectral intensities and shape. This is different from our finding for $\mathrm{Co}$ atoms in $\mathrm{Pt}_{3} \mathrm{Co} / \mathrm{KB}$ catalyst samples, where the chemical structure of Co changes gradually with annealing temperature. This could be a result of the more noble nature of Ir as compared to $\mathrm{CO}$ and/or due to the fact that different metal precursors were used for the two samples. 


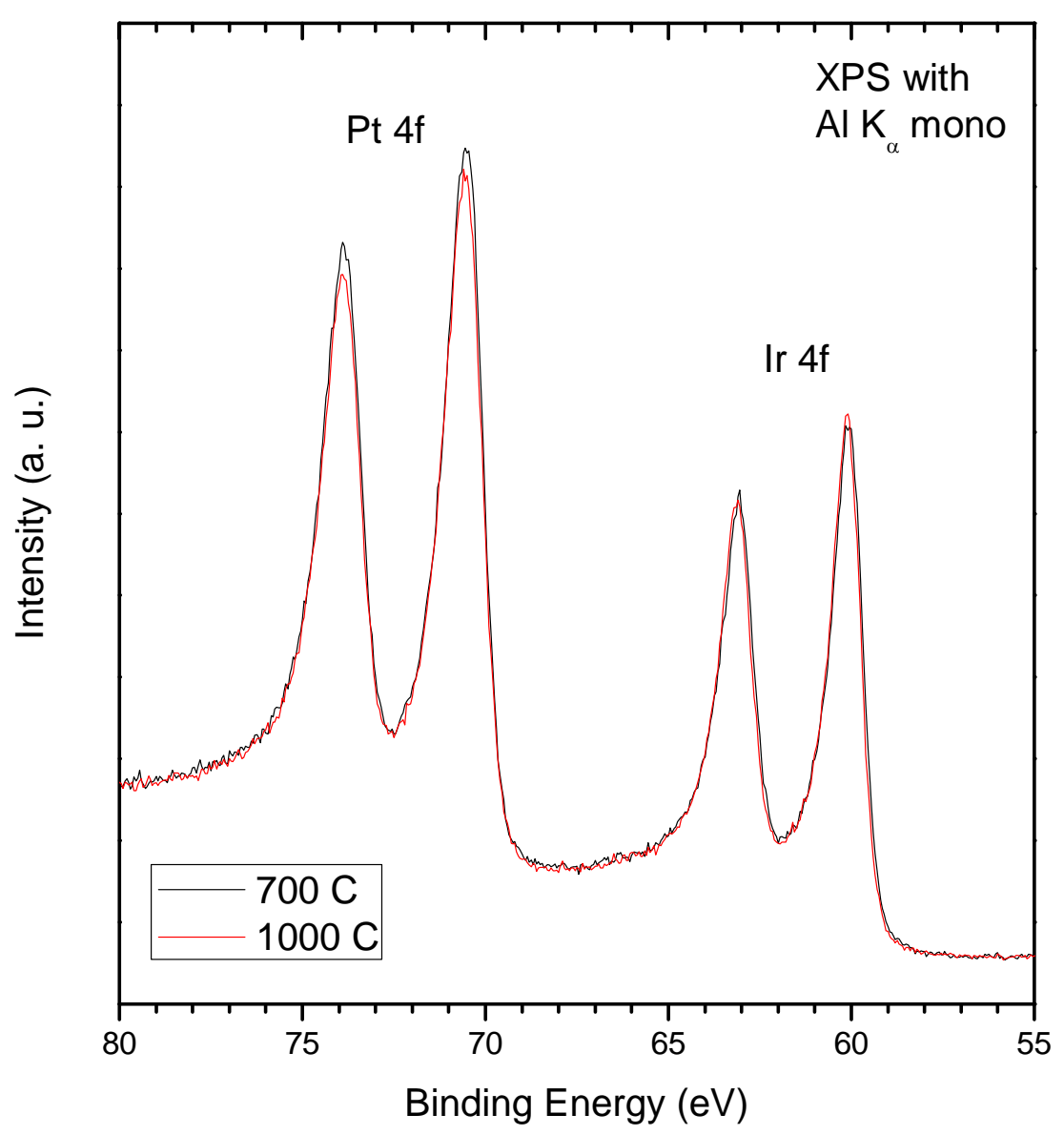

Fig. 40: Pt and Ir 4f XPS of PtIr/KB samples.

Fig. 41 shows $\mathrm{O} 1 \mathrm{~s}$ and $\mathrm{Pt} 4 \mathrm{p}_{3 / 2}$ XPS spectra of the $\mathrm{Pt}_{3} \mathrm{Co} / \mathrm{KB}$ samples and a curve fit with four Voigt functions. The fit was performed simultaneously for all four spectra, coupling a maximal number of fit parameters (e.g., peak positions and peak widths) for all spectra. This leads to very good overall agreement with the experimental results, with the largest deviations being observed for sample i. The main $\mathrm{O}$ 1s feature consists of at least two components: metal oxides (around $530 \mathrm{eV}$ ) and hydroxides (around $532 \mathrm{eV}$ ). Under similar sample preparation conditions, the sample annealed at lower temperature shows higher $\mathrm{O} 1 \mathrm{~s}$ intensity compared to that annealed at higher temperature. The $\mathrm{O} 1 \mathrm{~s}$ intensity of samples prepared at UTC is higher than that of samples prepared at the UNLV, especially for the metal oxides component, most likely due to additional oxidation during transit. Meanwhile, the Pt $4 \mathrm{p}_{3 / 2}$ feature also has two components: metallic Pt around $520 \mathrm{eV}$ and Pt oxides around $524 \mathrm{eV}$. The ratio between the two components does not change more than $20 \%$ for the different samples, i.e., significantly less than the variation in oxygen intensity. Therefore, the increase of the $\mathrm{O} 1 \mathrm{~s}$ intensity between samples prepared at the UTC and UNLV is interpreted as a result of oxidation of non-Pt metal components (likely Co in 


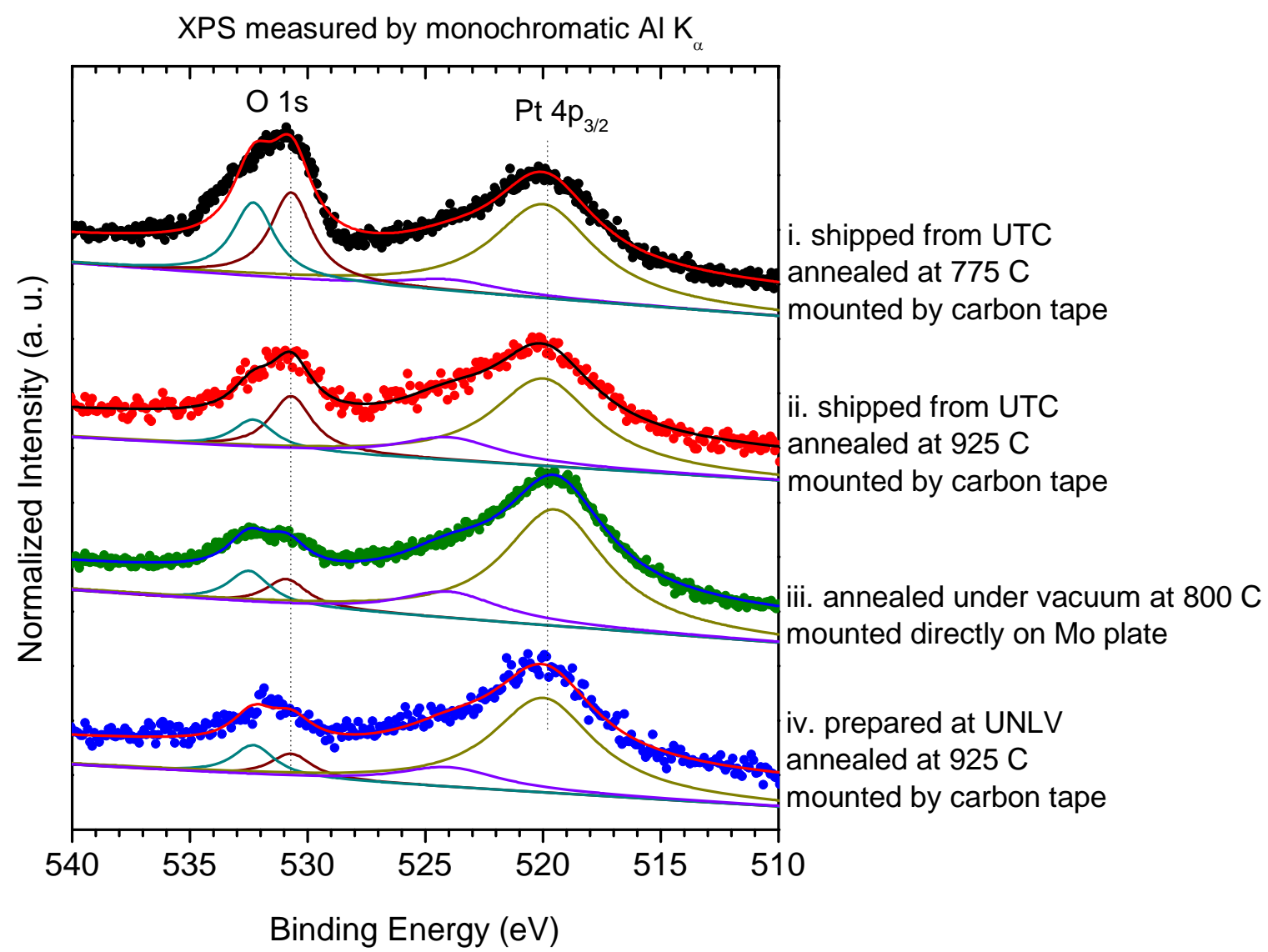

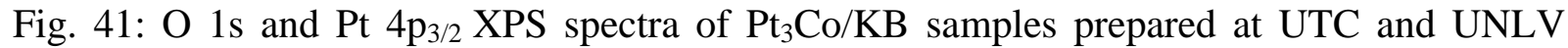
under different annealing temperatures and environments as listed (unless otherwise noted, samples were annealed under Ar flow). Spectra were normalized by the countrate at the lowbinding energy side of the $\mathrm{Pt} 4 \mathrm{p}_{3 / 2}$ peak.

this case) and/or adsorption of oxygen-containing species on the carbon black during shipping. Furthermore, the $\mathrm{O}$ 1s spectrum from the sample annealed under vacuum has been obtained insitu at a base pressure of 5E-10 mbar within two hours after the annealing process. Surprisingly, it shows a similar $\mathrm{O}$ 1s intensity as the sample annealed under Ar flow (at higher annealing temperature). This indicates that a reduction of the oxygen content in the annealing environment (i.e., vacuum vs. Ar flow) is helpful in reducing the oxygen content in/on the samples. However, even annealing in vacuum is not sufficient to fully remove the oxygen from the samples.

Fig. 42 shows the valence band structure of the various $\mathrm{Pt}_{3} \mathrm{Co} / \mathrm{KB}$ samples, as well as of a Co and Pt reference foil. The overall valence band structure of the catalyst samples is closer to that of Pt than that of Co, in particular in the spectral region between 3 and $6 \mathrm{eV}$. However, none of 


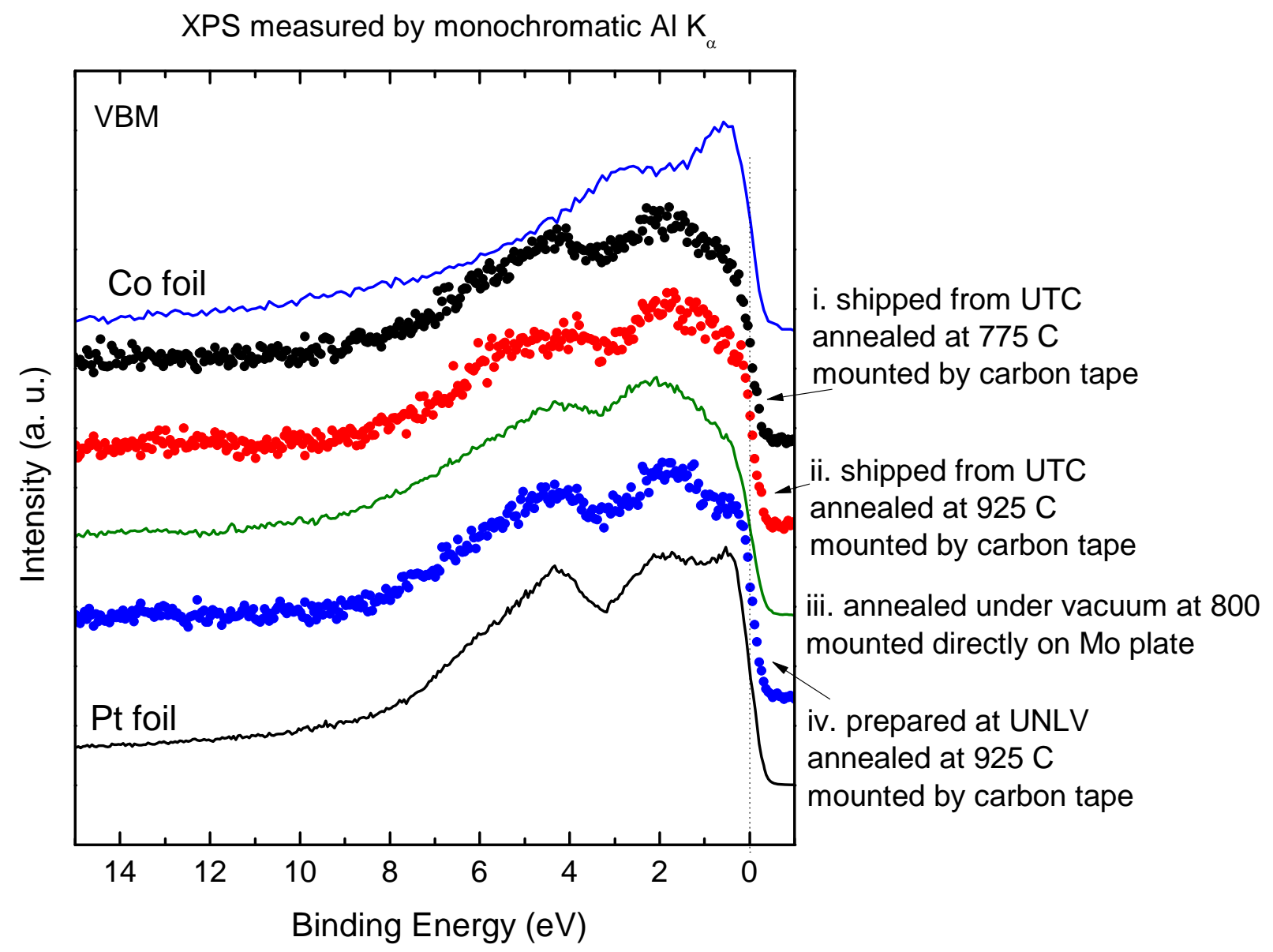

Fig. 42: $\mathrm{VBM}$ of $\mathrm{Pt}_{3} \mathrm{Co} / \mathrm{KB}$ samples prepared at UTC and UNLV under different annealing temperature and environments.

the catalyst samples exhibits the strong spectral intensity around $1 \mathrm{eV}$ found for both, the Pt and the Co reference. This indicates that the electronic valence structure of the catalysts is uniquely different from a simple superposition of Pt and Co spectral distributions. Further studies will need to be conducted to ascertain whether these differences are due to size quantization effects, surface adsorbates or oxidation, and structural surface-step effects. 


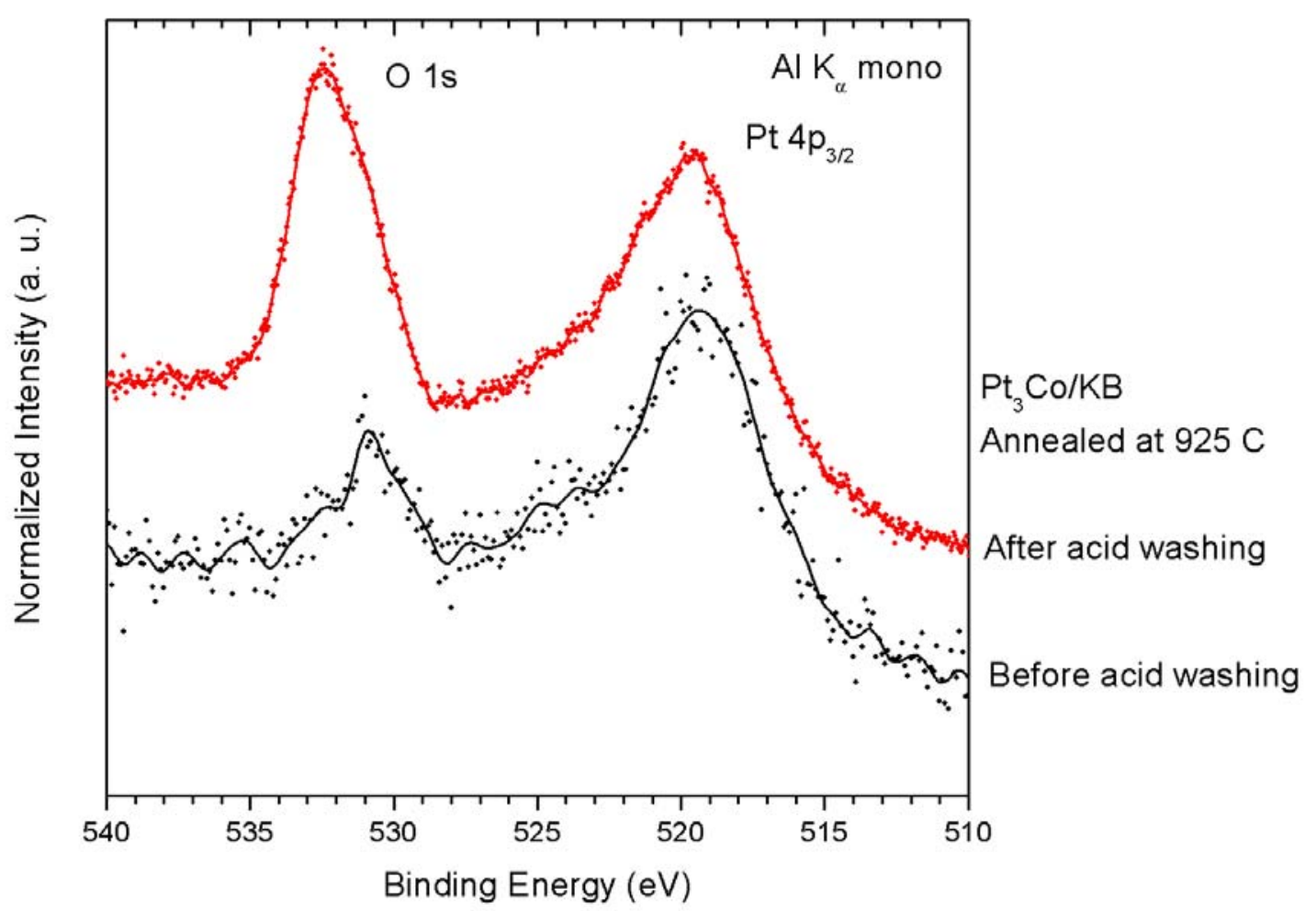

Fig. 43: $\mathrm{O} 1 \mathrm{~s}$ and $\mathrm{Pt} 4 \mathrm{p}$ spectra of $\mathrm{Pt}_{3} \mathrm{Co} / \mathrm{KB}$ catalysts before and after acid washing.

We have also studied the impact of acid washing on the electronic structure of Pt-based bimetallic nanoparticle catalysts on carbon black support $\left(\mathrm{Pt}_{3} \mathrm{Co} / \mathrm{KB}\right)$. The $\mathrm{Pt} 3 \mathrm{Co} / \mathrm{KB}$ sample, annealed at $925{ }^{\circ} \mathrm{C}$ following the UTC recipe, was soaked in $0.5 \mathrm{M} \mathrm{H}_{2} \mathrm{SO}_{4}$ (heated to $80{ }^{\circ} \mathrm{C}$ ) for 5 hours. Then, the acid-washed sample was rinsed several times with deionized water and dried in $\mathrm{N}_{2}$ atmosphere.

XPS survey spectra of the $\mathrm{Pt}_{3} \mathrm{Co} / \mathrm{KB}$ samples before and after acid washing (not shown) indicate that the dominant features in both spectra represent mainly $\mathrm{C}, \mathrm{Pt}$, and Co, as expected. The acidwashed sample shows a stronger $\mathrm{O}$ 1s signal, but no observable $\mathrm{S}$ signal. This indicates that the $\mathrm{O}$ signal is not likely from residual acid/sulfate, but probably stems from the rinsing procedure. The detailed $\mathrm{O} 1 \mathrm{~s}$ and $\mathrm{Pt} 4 \mathrm{p}$ XPS spectra of the $\mathrm{Pt}_{3} \mathrm{Co} / \mathrm{KB}$ samples before and after acid washing are shown in Fig. 43. The Pt $4 \mathrm{p}$ features in the two samples have similar spectral shape and intensity. A similar result was found in Pt 4f XPS spectra from the two samples (not shown). This indicates that the acid treatment does not induce a significant change in the local Pt environment, as expected. However, the $\mathrm{O} 1 \mathrm{~s}$ feature shows a substantial difference. The $\mathrm{O} 1 \mathrm{~s}$ feature of the acid-washed sample consists of at least two components, one around $531 \mathrm{eV}$ (metal oxide) and the other one around $532 \mathrm{eV}$ (hydroxide). The latter component is much stronger than that of the annealed sample, while the intensity of the former component does not change as 


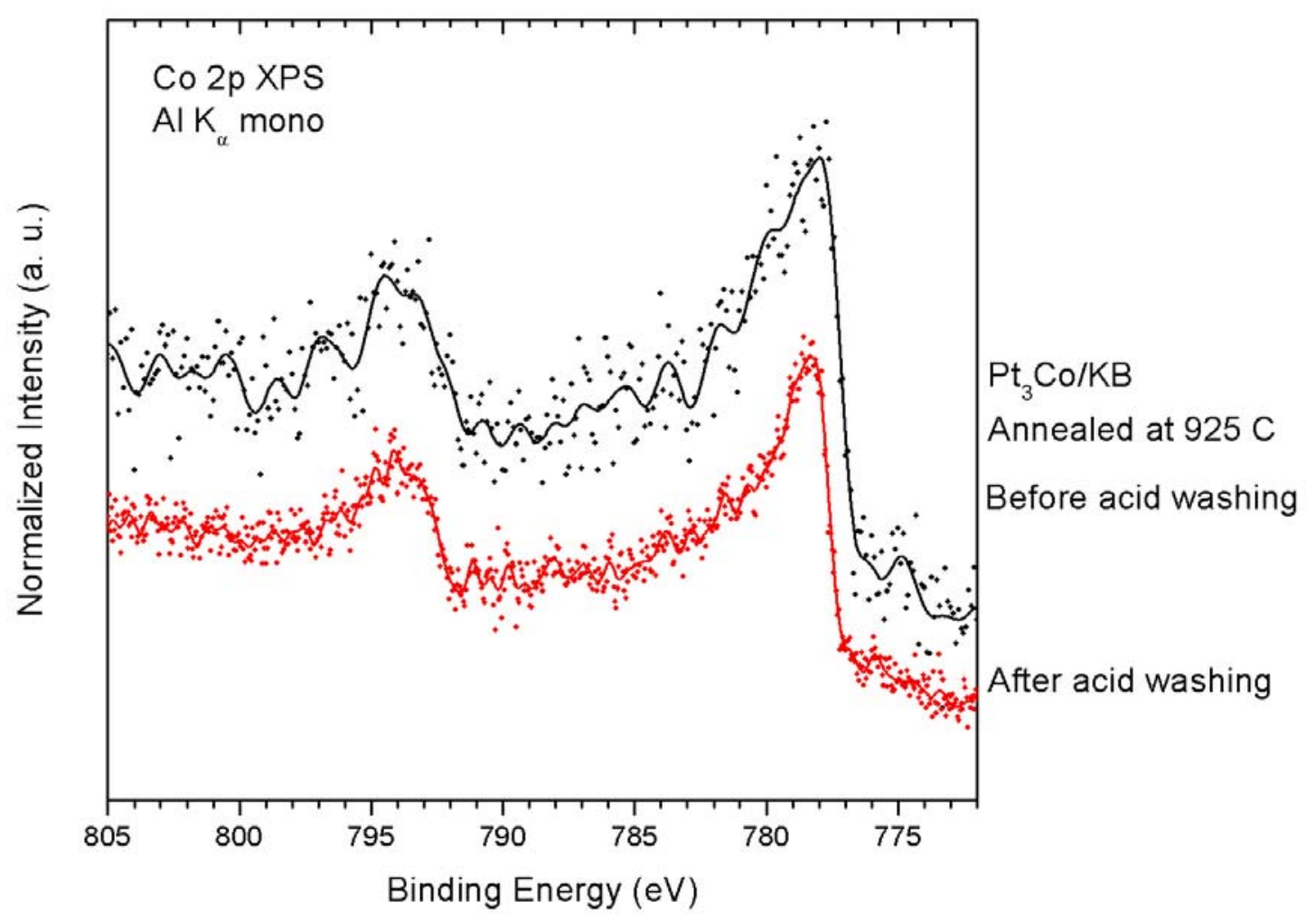

Fig. 44: Co 2p spectra of $\mathrm{Pt}_{3} \mathrm{Co} / \mathrm{KB}$ catalysts before and after acid washing. The solid line was obtained from a smoothing procedure and is intended as a guide to the eye only.

much. This is likely due to the adsorption of hydroxides on the sample surface after acid treatment.

The most surprising observation is found in the Co 2p XPS spectra, shown in Fig. 44. The acidwashed sample shows similar Co 2p signals as the annealed sample. This is in contrast to the earlier findings on "off-the-shelf" samples received in 2007, which were also acid-treated (before long-term storage in air) and showed no Co signal on the surface.

To better understand the impact of Ir admixture on the electronic and chemical surface structure, we studied Pt-based tri-metallic nanoparticle catalysts on graphitized Vulcan XC-72 carbon support $\left(\mathrm{Pt}_{2} \mathrm{IrCo} / \mathrm{VC}\right)$. Three $\mathrm{Pt}_{2} \mathrm{IrCo} / \mathrm{VC}$ samples were annealed at 926, 1000 , and $1100{ }^{\circ} \mathrm{C}$, respectively, in the lab of our collaboration partners at UTC.

Fig. 45 shows XPS of the $\mathrm{O} 1 \mathrm{~s}$, Pt and Ir $4 \mathrm{p}_{3 / 2}$ lines of three samples. The spectra were normalized to the background on the lower binding energy side for easier comparison. Compared to the Ir $4 \mathrm{p}_{3 / 2}$ feature, the Pt line shows a decrease with increasing annealing temperature. Similar to other nanoparticle catalysts we studied before, the $\mathrm{O} 1 \mathrm{~s}$ feature consists of at least two components: metal oxides (around $530 \mathrm{eV}$ ) and hydroxides (around $532 \mathrm{eV}$ ). The line shape of the $\mathrm{O} 1 \mathrm{~s}$ feature from the sample annealed at $926^{\circ} \mathrm{C}$ is quite different from the other two spectra. The samples annealed at higher temperatures show lower intensity around $530 \mathrm{eV}$ but stronger 
intensity around $532 \mathrm{eV}$. This indicates that at higher annealing temperature, metal oxides can be further reduced.

Fig. 46 shows Co 2p XPS spectra of the three samples. The spectra were normalized in the same fashion as those in Fig. 45. All spectra show that Co atoms in/on the samples are present in at least two chemical environments: metallic and oxides. The spectral intensity of the Co oxide component decreases at higher annealing temperatures $\left(1000\right.$ and $\left.1100{ }^{\circ} \mathrm{C}\right)$. This observation is consistent with the decrease of the metal oxide component in the $\mathrm{O} 1 \mathrm{~s}$ feature discussed above. Therefore, a higher annealing temperature is preferred to transform metal precursors to their metallic form, but also appears to change the $\mathrm{Pt} / \mathrm{Ir}$ ratio at the surface.

The (a) Pt and Ir 4f XPS spectra of three samples and (b) their difference spectra are shown in Fig. 47. The XPS spectra were normalized to the background on the lower binding energy side $(58 \mathrm{eV})$ for quantitative comparison and calculation of difference spectra. The intensities of the Pt $4 \mathrm{f}$ features decrease gradually with increasing annealing temperature. We speculate that this could result from Pt agglomeration. It is confirmed by UTC that the average particle size is 4.8, 5.4, and $6.2 \mathrm{~nm}$ for the samples annealed at 926,1000 , and $1100{ }^{\circ} \mathrm{C}$, respectively. Since the inelastic mean free path (IMFP) of electrons at this kinetic energy is only about $1.5 \mathrm{~nm}$ (in Pt and Ir), the increase of particle size reduces the number of atoms that contribute to the XPS peak intensity. If Pt atoms are distributed evenly in the particles, the increase of particle size reduces the Pt $4 \mathrm{f}$ spectral intensity, as seen here. The intensity decrease of the Pt $4 \mathrm{f}$ features is larger as

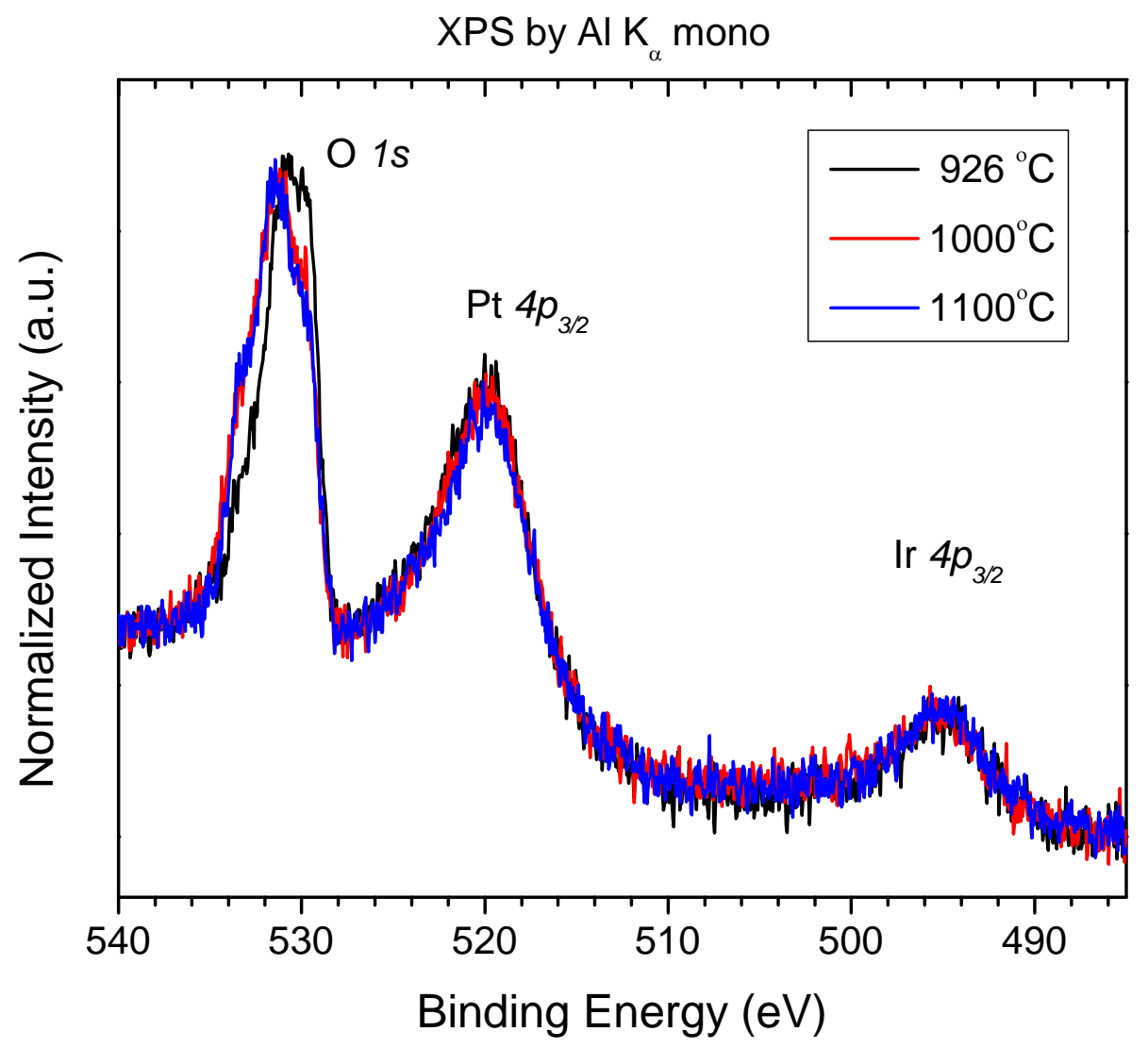

Fig. 45: O 1s, Pt $4 \mathrm{p}_{3 / 2}$ and Ir $4 \mathrm{p}_{3 / 2}$ spectra from three $\mathrm{Pt}_{2} \mathrm{IrCo} / \mathrm{VC}$ samples, annealed at 926 , 1000 , and $1100{ }^{\circ} \mathrm{C}$, respectively. 


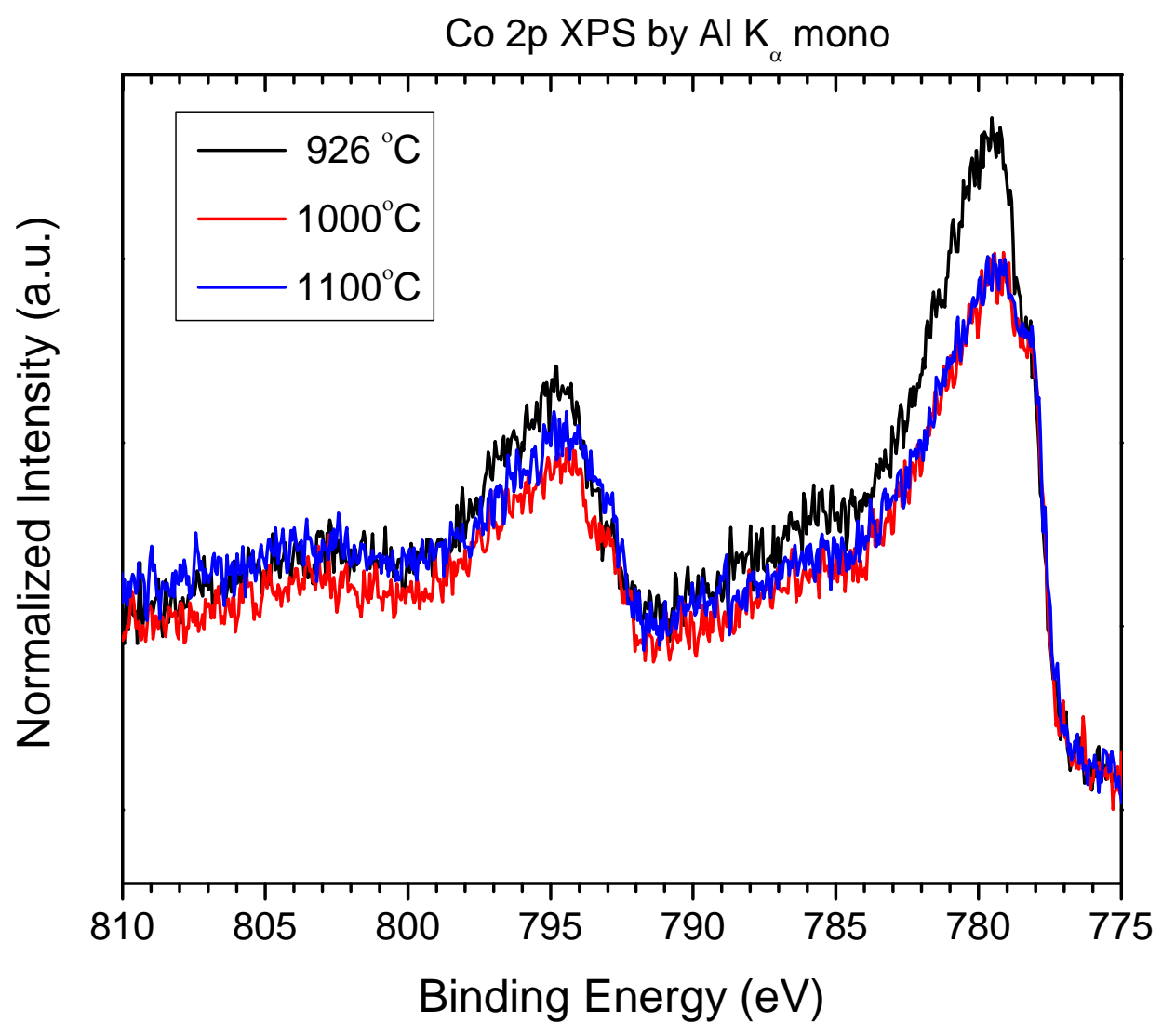

Fig. 46: Co 2p spectra from three $\mathrm{Pt}_{2} \mathrm{IrCo} / \mathrm{VC}$ samples, annealed at 926,1000 , and $1100{ }^{\circ} \mathrm{C}$, respectively.

the particle size changes from 4.8 to $5.4 \mathrm{~nm}$ than for the change from 5.4 to $6.2 \mathrm{~nm}$. This indicates that the Pt atom distribution in the particles changes when the sample is annealed at $1100{ }^{\circ} \mathrm{C}$, i.e., Pt atoms migrate towards the surface of the particles. This combination of agglomeration and surface segregation is even more pronounced for the Ir atoms. When comparing the samples annealed at 926 and $1000{ }^{\circ} \mathrm{C}$, the intensities of the Ir $4 \mathrm{f}$ features decrease slightly. For the annealing step from 1000 to $1100{ }^{\circ} \mathrm{C}$, the intensities increase significantly. Since the change between 926 and $1000{ }^{\circ} \mathrm{C}$ is much less pronounced than in the Pt case, we speculate that the intensity reduction due to agglomeration is overcompensated by the intensity increase associated with surface segregation. For higher temperatures, the segregation is clearly dominant. This suggests that the Ir surface segregation happens at lower temperature than that of Pt. Note that a segregation of both $\mathrm{Pt}$ and $\mathrm{Ir}$ in a $\mathrm{Pt}_{2} \mathrm{IrCo}$ nanoparticle is synonymous with the formation of a core with increased Co density, i.e., in agreement with earlier findings of a reduced Co concentration at the nanoparticle surface. 
(a) XPS of $\mathrm{Pt}_{2} \mathrm{IrCo} / \mathrm{C}$ by $\mathrm{Al} \mathrm{K}_{\alpha}$ mono

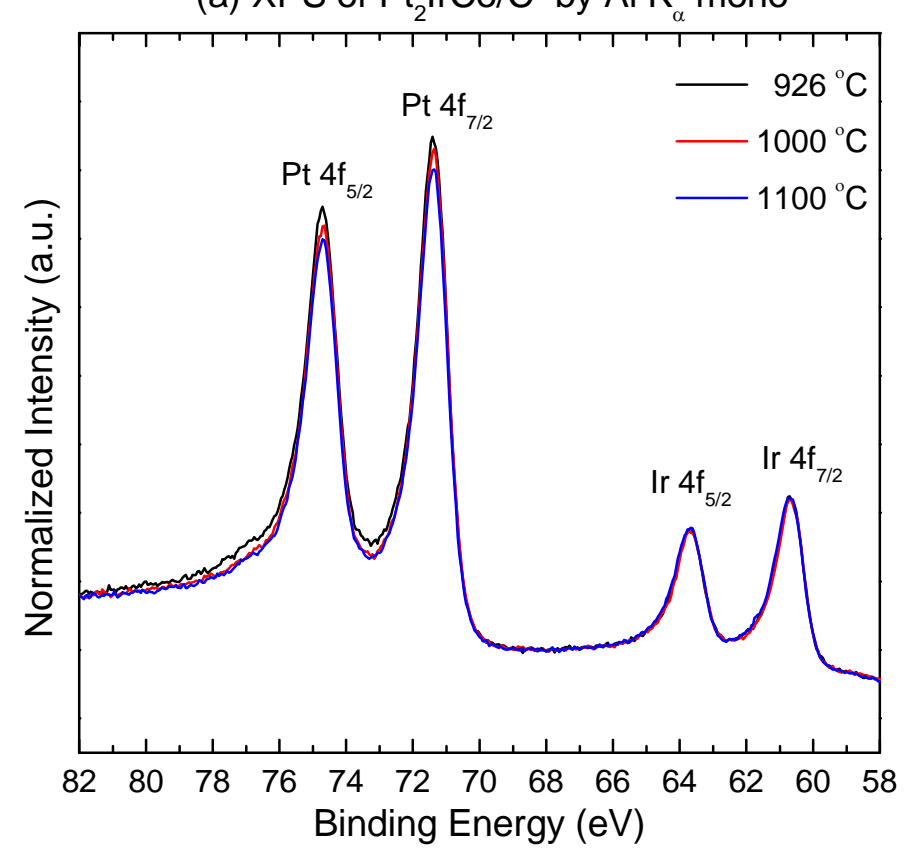

(b) Pt and Ir 4f Difference Spectra

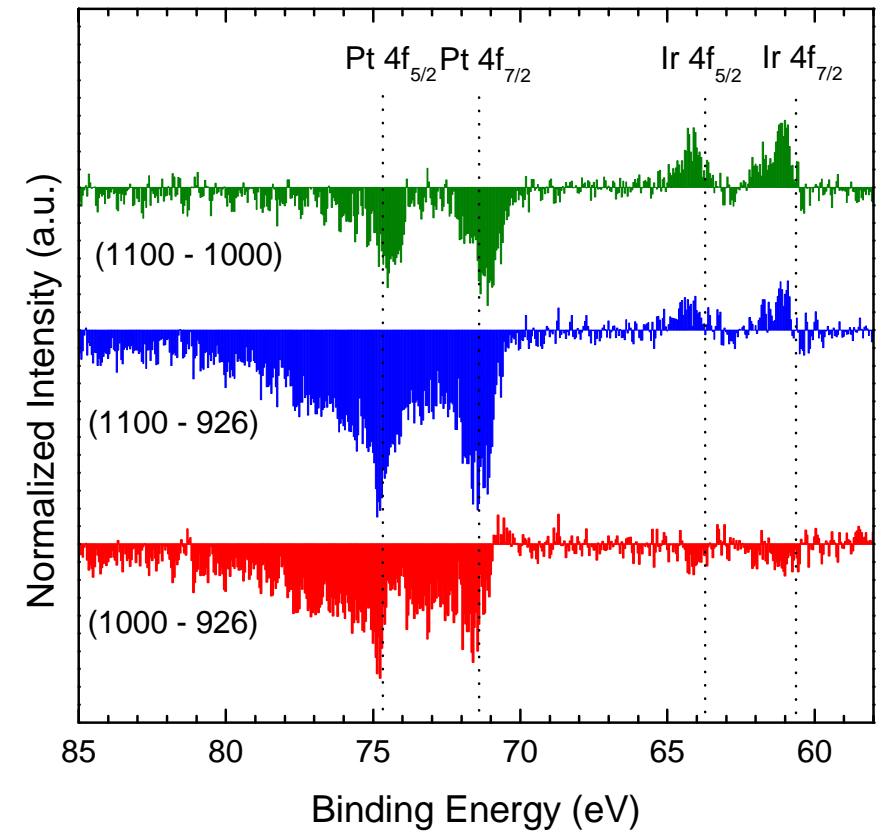

Fig. 47: (a) Pt and Ir 4f XPS spectra from three $\mathrm{Pt}_{2} \mathrm{IrCo} / \mathrm{VC}$ samples, annealed at 926, 1000, and $1100{ }^{\circ} \mathrm{C}$, respectively. (b) Difference spectra of the Pt and Ir $4 \mathrm{f}$ spectra. 


\section{Presentations and Publications:}

1. C. Heske, "Hydrogen Fuel Cells and Storage Technology", presentation to the FreedomCAR

2. Initiative, November 10, 2005.

3. C. Heske, “X-ray Spectroscopy of Buried Things: Interfaces, Liquids and Dirty Powders”, Stanford Synchrotron Radiation Laboratory, February 27, 2006.

4. C. Heske, "Hydrogen Fuel Cells and Storage Technology”, Ford Motor Company, March 29, 2006.

5. C. Heske, "Hydrogen Fuel Cells and Storage Technology Project at UNLV”, DOE Hydrogen Review Meeting, Arlington, VA, May 17, 2006.

6. C. Heske, "Surface and Interface Analysis at UNLV”, United Technologies Company Fuel Cells, Hartford, CT, June 23, 2006.

7. C. Heske, “UNLV Research Foundation - overview of hydrogen research projects”, United Technologies Research Center, Hartford, CT, June 23, 2006.

8. C. Heske, “Hydrogen Fuel Cells and Storage Technology Project at UNLV”, United Technologies Research Center, Hartford, CT, June 23, 2006.

9. C. Heske, "Surface and interface analysis in Las Vegas", Seminar Experimentelle Physik II, University of Würzburg, Germany, July 20, 2006.

10. C. Heske, "How to reveal the chemical and electronic properties of interfaces, buried layers, and liquids with soft x-ray spectroscopy”, Materials Science and Engineering Department, Stanford University, August 31, 2006.

11. L. Weinhardt, FCAST Project Meeting, November 16, 2006.

12. C. Heske, "The surface and interface analysis "tool chest” at UNLV”, Group seminar Prof. T. Moustakas, Boston University, November 17, 2006.

13. C. Heske, "How to reveal the chemical and electronic properties of interfaces, buried layers, and liquids with soft x-ray spectroscopy”, Physics Department, Boston University, December 8, 2006.

14. C. Heske, "Hydrogen Fuel Cells and Storage Technology", FCAST Project Presentation (Storage only) to the Technical Team Meeting, FreedomCAR, Detroit, December 19, 2006.

15. C. Heske and B. Naduvalath, DOE Hydrogen Review Meeting, Arlington, VA, May 2007.

16. C. Heske, "Understanding and Optimizing Surfaces and Interfaces in Energy Conversion Devices”, 2007 Annual Energy Symposium, UNLV, August 16, 2007 (invited oral).

17. I.C. Tran, R. Felix, L. Weinhardt, M. Bär, and C. Heske, “Ti-coated Single-walled Carbon Nanotubes for Hydrogen Storage: A Spectroscopic and Microscopic Study”, 2007 Fall MRS Meeting, Boston, MA, Nov. 2007 (poster).

18. C. Heske, "Soft x-rays and the electronic structure of buried things - interfaces, liquids, and below dirty surfaces”, Materials Science Institute, University of Oregon, Eugene, February 26, 2008.

19. C. Heske, Presentation during the site visit of Jesse Adams, DOE Golden Field Office, on March 14, 2008.

20. C. Heske and N. Balakrishnan, "Hydrogen Fuel Cells and Storage Technology Project”, Presentation to the DOE Hydrogen Storage Tech Team, FreedomCAR, Detroit, April 17, 2008.

21. C. Heske and N. Balakrishnan, "Hydrogen Fuel Cells and Storage Technology Project", Poster presentation at the DOE Program Review Meeting, Arlington, June 9, 2008.

22. Y. Zhang, L. Weinhardt, T. Hofmann, M. Bär, C. Heske, and T.T. Aindow, "The chemical and electronic structure of platinum-based nanoparticle catalysts for PEM fuel cells”, 
American Chemical Society Western Regional Meeting, Las Vegas, Sep. 24, 2008 (invited oral).

23. Roberto Felix, Ich Tran, Lothar Weinhardt, Marcus Bär, Timo Hofmann, Yufeng Zhang, and Clemens Heske, "Study of the Interaction between Hydrogen and Carbon-based Nanomaterials”, 2008 MRS Fall Meeting, Boston, MA, Nov. 2008 (poster, won best poster award).

24. Ich Tran, "Microscopic and Spectroscopic Studies of Metal Deposition on Carbon-based Materials”, Ph.D. thesis defense presentation, Department of Chemistry, UNLV, November 21, 2008.

25. I. Tran, R. Felix, Y. Zhang, M. Bär, C. Heske, and L. Weinhardt, "Microscopic and Spectroscopic Studies of Metal Deposition on Carbon Nanomaterials”, New Diamond and Nano Carbons Conference (NDNC 2009), June 7-11, Traverse City, MI (poster).

26. I. Tran, R. Felix, M. Bär, L. Weinhardt, Y. Zhang, and C. Heske, “Oxidation of Titaniumdecorated Single-Walled Carbon Nanotubes and subsequent reduction by Lithium”, J. Am. Chem. Soc. 132, 5789-5792 (2010).

27. C. Heske, "Using soft $\mathrm{x}$-rays to look at surfaces and interfaces for energy conversion and storage”, National Renewable Energy Lab (NREL), June 21, 2010.

28. C. Heske, "Wie man mit Röntgenspektroskopie energierelevante Materialien und Systeme verstehen und verbessern kann“, Organisch-Chemisches Kolloquium im Sommersemester 2010, Karlsruhe Institute of Technology, July 2, 2010.

29. C. Heske, "Using soft x-rays to understand and improve materials for energy conversion devices“, Department of Chemistry, University of California Santa Cruz, September 29, 2010.

30. C. Heske, "Using soft x-rays to optimize materials for energy conversion devices”, Department of Physics, Renmin University, Beijing, China, Oct. 10, 2010.

31. C. Heske, "Using soft x-rays to understand and optimize materials for energy conversion", Department of Physics, Hanoi University of Science and Technology, Hanoi, Vietnam, Nov. 1, 2010.

Manuscripts in preparation:

32. Y. Zhang et al., "Electronic structure of Pt-based nanocatalysts", in preparation for J. Phys. Chem.

33. I. Tran et al., "Oxidation behavior of metal adatoms on carbon nanomaterials”, in preparation for J. Phys. Chem.

34. I. Tran et al., "Local atomic and electronic surface structure of carbon nanomaterials, metal adatoms, and adsorbed molecular species", in preparation for Surface Science.

35. Felix, R. et al., "Temperature Dependence of Tungsten-mediated Hydrogen Dissociation monitored by Hydrogen Chemisorption on Single-Walled Carbon Nanotubes”, in preparation for J. Vacuum Science and Technology. 


\section{ATTACHMENT B}

Task \#11: “X-ray Spectroscopy Studies for Hydrogen Storage - Study of Interactions in the System: Carbon Nanotubes - Transition Metals - Hydrogen”

Investigators: O. Hemmers, D. Lindle, W. Stolte, K. Lipinska-Kalita

Project Start Date: October 1, 2005

Project End Date: September 30, 2007

\section{Project Background.}

Finding which materials are ideal candidates for room-temperature, high wt. \%, reversible hydrogen storage is currently a major interdisciplinary challenge spanning material science, chemistry and physics. The U.S. Department of Energy (DOE) has established a multistage target for hydrogen storage capacity for fuel-cell applications. The design targets for an on-board hydrogen storage system are 6 wt \% by 2010, and 9 wt \% by 2015, at moderate temperatures and pressures (0-100 oC and 1-10 atm.). Among the currently available candidate storage materials, none is capable of meeting the DOE criteria for personal transportation vehicles. Hydrogen can be stored in solid materials either in atomic or molecular form. Storage of hydrogen in molecular form has an advantage in that molecular hydrogen has fast kinetics.

However, its bonding is very weak and desorption can take place at low temperatures. Materials capable of storing hydrogen with high gravimetric and volumetric density, operating under ambient thermodynamic conditions, and exhibiting fast hydrogen sorption kinetics are essential for practical applications. In search for alternate hydrogen storage candidates, considerable attention has been focused on porous materials such as clathrates, zeolites, carbon nanotubes, fullerenes and nanocomposites.

Carbon Nanotubes (CNTs) - Single-wall carbon nanotubes (SWCNTs) have particularly been promoted as promising materials for hydrogen storage due to their light mass density, and good interaction with hydrogen molecules that could be stored inside the tube cavities, in the interstitial sites of nanotube bundles, or between the graphitic layers. Theoretical studies considered perfectly structured SWCNT's while numerous experiments were performed with defective CNT's which is probably why research groups reported very different results on capacity of hydrogen storage in nanotubes. So far, the most reported and partially reliable hydrogen uptake values for multiwall carbon nanotubes are around 1-3 wt \%.

Interaction Transition Metals - Hydrogen - Hydrogen is commonly known to interact with metal surfaces in one of two ways: it is either physisorbed molecularly or chemisorbed atomically due to charge transfer from the metal surface to the antibonding orbital of the $\mathrm{H} 2$ molecule. The binding energy of hydrogen in the physisorbed state is of the order of a few meV's, while that in the chemisorbed state is of the order of a few eV's. In the former case, desorption of hydrogen occurs at very low temperatures and is not ideal for storage under ambient thermodynamic conditions. In the latter case, the binding can be strong and desorption would require high temperatures. An ideal form of binding would be in between physisorption and chemisorption. In this energy range the $\mathrm{H} 2$ molecular bond would not break, but would get slightly elongated due to charge polarization and/or orbital overlap. It was shown that a metal cation can bind to multiple hydrogen atoms in molecular form. In this case the ability of the 
metal cation to transfer electrons to the antibonding state of the $\mathrm{H}_{2}$ molecule is diminished, and hence, the $\mathrm{H}_{2}$ molecules are bound by the charge polarization mechanism. The binding energies of the hydrogen molecules are usually small $(\sim 0.2 \mathrm{eV})$ and the maximum number of $\mathrm{H}_{2}$ molecules that can be stored by a single metal cation only depends on the steric condition.

Interaction Transition Metals - Carbon Nanotubes - CNTs are ideal templates for fabricating a variety of nanowire materials. The unique structures of nanotubes provide confined platforms for physical processes and chemical reactions. In principle, nanowires of any metal can be obtained by simply depositing metal onto nanotubes. However, depending on the metal type, formation of a continuous and uniform coating on nanotubes or decorations of isolated discrete particles can be observed and this is determined by the strength of the CNT - metal interaction. Only a limited number of metals including titanium have strong interactions with nanotubes, and allow them to form continuous structures on a nanotube surface. Theoretical studies also show that the interaction of transition metal (TM) atoms with SWNTs depends on the specific transition metal, resulting from a variation of the hybridization between carbon $p_{z}$ and the transition metal $d$ orbitals. A quantitative measure of the binding energies between various metal atoms and SWNTs has not been carried out at the present time. Nevertheless, based on the observed sticking behavior of metals on the nanotubes and the resulting metal nanostructures, it was tentatively suggest that: $E_{b}(\mathrm{Ti})>E_{b}(\mathrm{Ni})>E_{b}(\mathrm{Pd})>E_{b}(\mathrm{Fe})>E_{b}(\mathrm{Al})>E_{b}(\mathrm{Au})$. In particular, it has been experimentally proven that the evaporation of Ti leads to the formation of a continuous and uniform coating on nanotubes even at a nominal thickness below $1 \mathrm{~nm}$ and was attributed to strong interaction between Ti and SWNTs, high Ti nucleation density, and a low diffusion rate of $\mathrm{Ti}$ atoms on nanotubes.

In order to take advantage of both of these forms of hydrogen-metal bonding one must chose a suitable substrate on which a transition metal atom ( $\mathrm{Sc}, \mathrm{Ti}, \mathrm{V}, \mathrm{Cr}, \mathrm{Mn}, \mathrm{Fe}, \mathrm{Co}, \mathrm{Ni}$ ), through charge transfer, can remain in a positively charged state and hence bind a large number of hydrogen atoms. In addition, early transition metal atoms ( $\mathrm{Sc}, \mathrm{Ti}$, and $\mathrm{V}$ ) have nearly empty $d$ orbitals. Thus, the interaction of $\mathrm{H}_{2}$ molecules with unfilled $d$ orbitals can lead to intermediate binding energies, between physisorption and chemisorption energies.

\section{Comparison of the actual accomplishments with the goals and objectives of the project.}

\section{Project Goals}

The outcomes of our project are expected to advance our fundamental understanding of dissociative and molecular chemisorption of hydrogen in nanostructures based on the combination of carbon nanotubes (CNT) and a transition metal (TM), and will be a key in helping to develop novel materials for hydrogen storage. The transition metal selected for these studies was Titanium (Ti).

- understand, from a fundamental point of view, the interaction between the host material (the CNT - Ti system) and hydrogen; there is currently no systematic experimental work to address this subject

- advance the understanding of the nature of CNT - Ti and CNT - Ti - hydrogen interactions

- shed light on the manner in which the $\mathrm{CNT}$ - Ti - hydrogen interactions can be altered or tuned to increase the hydrogen storage capabilities 
- Quantify the binding energies between particular Ti atoms and CNTs and between Ti and hydrogen; no experimental data exists so far.

\section{Project Accomplishments}

Systems composed of single and multiwall carbon nanotubes deposited on Ti-substrates were investigated using X-ray absorption and emission spectroscopy using synchrotron radiation at the Advanced Light Source of Lawrence Berkeley National Laboratory. In addition, images of the nano-tube materials were taken using a Zeiss electron microscope at the molecular foundry of Lawrence Berkeley National Laboratory.

3. Summary of project activities (Include, if applicable, facts, figures, analyses, and assumptions used during the life of the project to support the conclusions).

The project was based on a recent theoretical study that has proposed an entirely new concept for storing hydrogen in its molecular form using carbon nanotubes combined with transition metal (Ti) atoms. We intended to experimentally verify this new concept and perform fundamental studies of bonding in the system SWCNT-Ti-Hydrogen.

\section{- Original hypotheses.}

Our leading idea was to experimentally explore the ways in which carbon nanotubes can be used as a backbone or matrix to which Ti atoms could be combined in order to construct new adsorbents capable of repetitively storing and releasing large amounts of hydrogen at close to ambient pressure and temperature conditions. The phenomenon that a single Ti atom absorbed on hexagonal phase of a SWNT can bind up to eight hydrogen molecules is a very general and novel result of theoretical work and could hold for a very large number of nanotubes and for other carbon-based nanoclusters. In a first-principles study it was demonstrated that a single $\mathrm{Ti}$ atom coated on a single-walled nanotube (SWNT) can bind up to four hydrogen molecules. The first $\mathrm{H}_{2}$ adsorption is dissociative with no energy barrier while the other three adsorptions are molecular with significantly elongated $\mathrm{H}-\mathrm{H}$ bonds. At high Ti coverage a SWNT can strongly adsorb up to $8 \mathrm{wt} \%$ of hydrogen. In addition, high-temperature quantum molecular dynamics simulations showed that these systems are stable and exhibit associative desorption of $\mathrm{H}_{2}$ upon heating, another requirement for reversible storage. Such an unusual and complex bonding is generated by the concerted interaction among $\mathrm{H}$, Ti, and SWNT. The mechanism of the bonding is explained by a unique hybridization between Ti-d, hydrogen $\sigma^{*}$ antibonding, and SWNT C- $p$ orbitals.

\section{- $\quad$ Approaches used.}

The key experimental technique used was a custom-built X-Ray Photon Emission Spectroscopy set-up and a X-Ray Absorption Spectroscopy set-up. In X-Ray Photon Emission spectroscopy, and $\mathrm{x}$-ray photon is absorbed by the target sample and removes a 1s electron from the target (here Ti). Next $2 p$ and $3 p$ electrons fall into the created $1 \mathrm{~s}$ vacancy and emit X-ray photons. A vacuum system with an X-ray spectrometer acts as the detector. The X-ray photons create a spectrum of lines that can be studied in regards to their intensities and positions. X-Ray Photon Emission spectroscopy supplies information about: (1) the amount of hydrogen that can be adsorbed on carbon nanotubes deposited on a Ti substrate; (2) how often the hydrogen 
adsorption/desorption cycle can be repeated; (3) whether an input of heat is needed for hydrogen cycling; (4) and to what degree are the Ti coated nano tubes stable.

An X-Ray Emission Setup as well as an X-Ray Absorption Setup were modified or custom-designed and built by the PIs at the Advanced Light Source of Lawrence Berkeley National Laboratory. In particular we designed and built: (1) a sample holder with heating capabilities for Near-Edge X-Ray Absorption Fine Structure spectroscopy (NEXAFS) and for Xray Emission Spectroscopy (XES) studies; (2) modified the exisiting NEXAFS experimental chamber; (3) a new crystal bender for XES setup to allow for experiments near the Ti 1s edge; (4) also the data acquisition for the XES system was upgraded. A number of compounds of different purity have been selected, purchased, and measured using X-ray absorption techniques, including indispensable reference materials and carbon nanotubes such as single wall carbon nanotubes - SWCNT and multiwall carbon nanotubes - MWCNT.
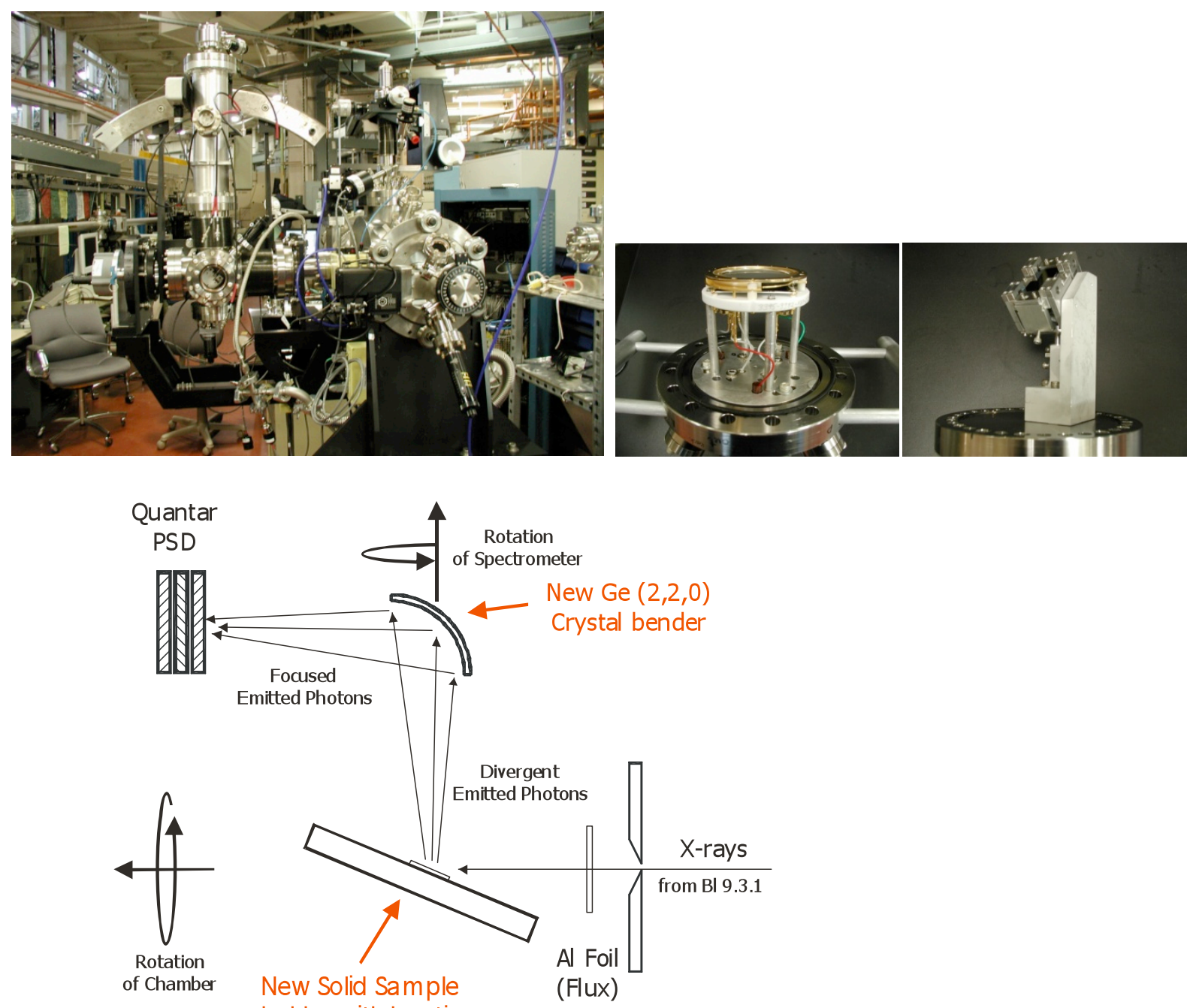

Figure 1. Custom- built X-Ray Emission Setup for studies of the interaction of carbon nanotubes deposited on a Ti substrate with molecular hydrogen. 

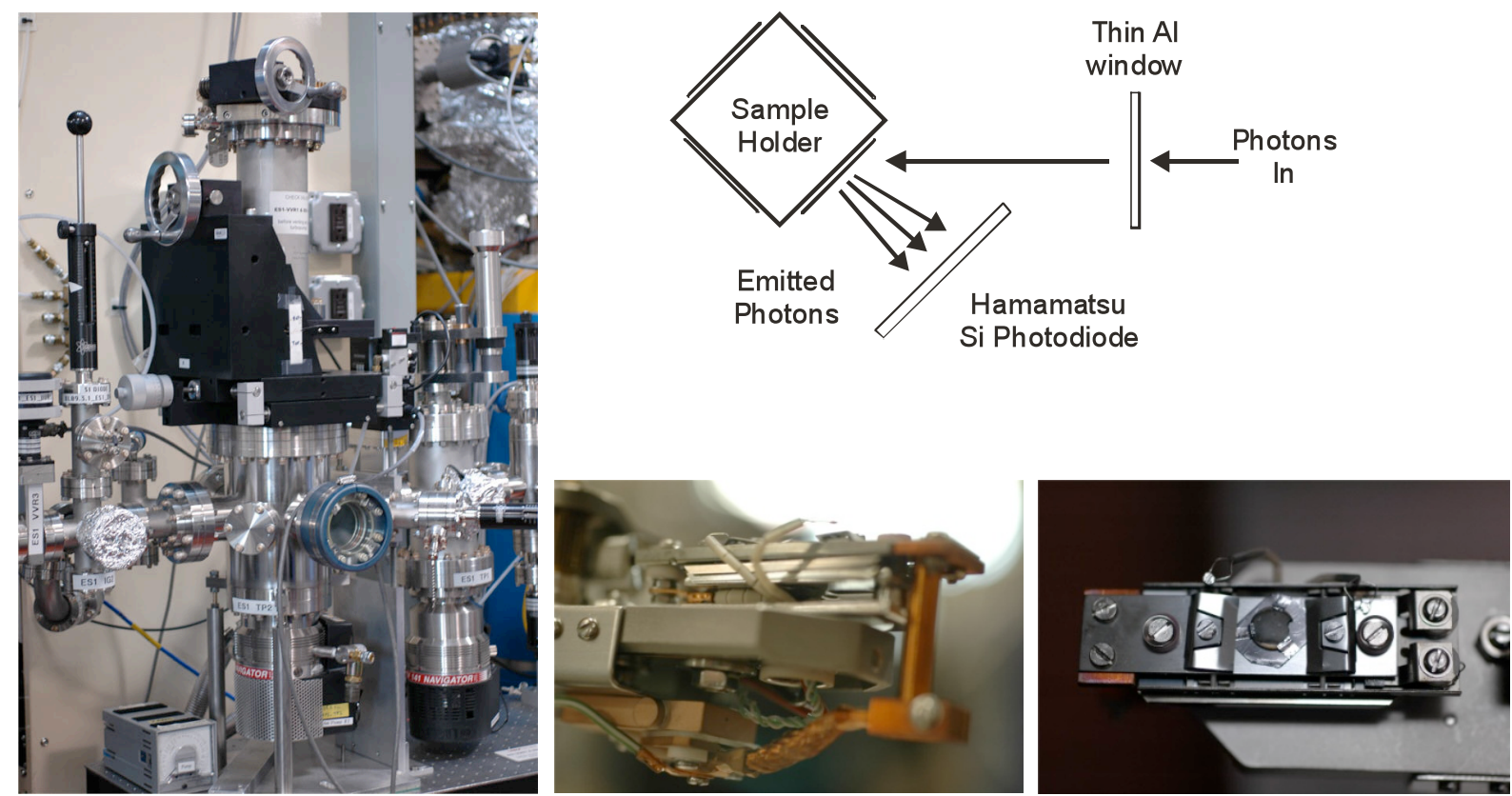

Figure 2. Custom- built X-Ray Absorption Setup for studies of the interaction of carbon nanotubes deposited on a Ti substrate with molecular hydrogen.

The project fostered a new collaboration with the Materials Science and Technology Division of Lawrence Livermore National Laboratory that specializes in fabrication and deposition of CNTs on different substrates. They fabricated several CNTs samples, deposited on silica wafers and titanium as substrates. These samples were then used in X-ray absorption studies.

We have also started collaboration with the Center for X-Ray Optics (CXRO) at LBNL that has experience with and equipment for metal vapor deposition. In the frame of this collaboration we fabricated thin film Ti coatings (from fractional mono-layers to multi-layers) on CNTs arrays, SWCNT and MWCNT powders deposited on different substrates.

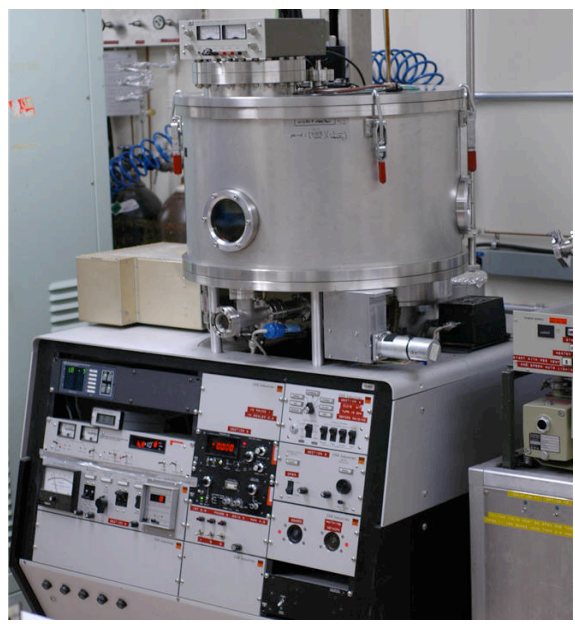

Figure 3. Metal Vapor Deposition System of the Center for X-Ray Optics at Lawrence Berkeley National Laboratory 


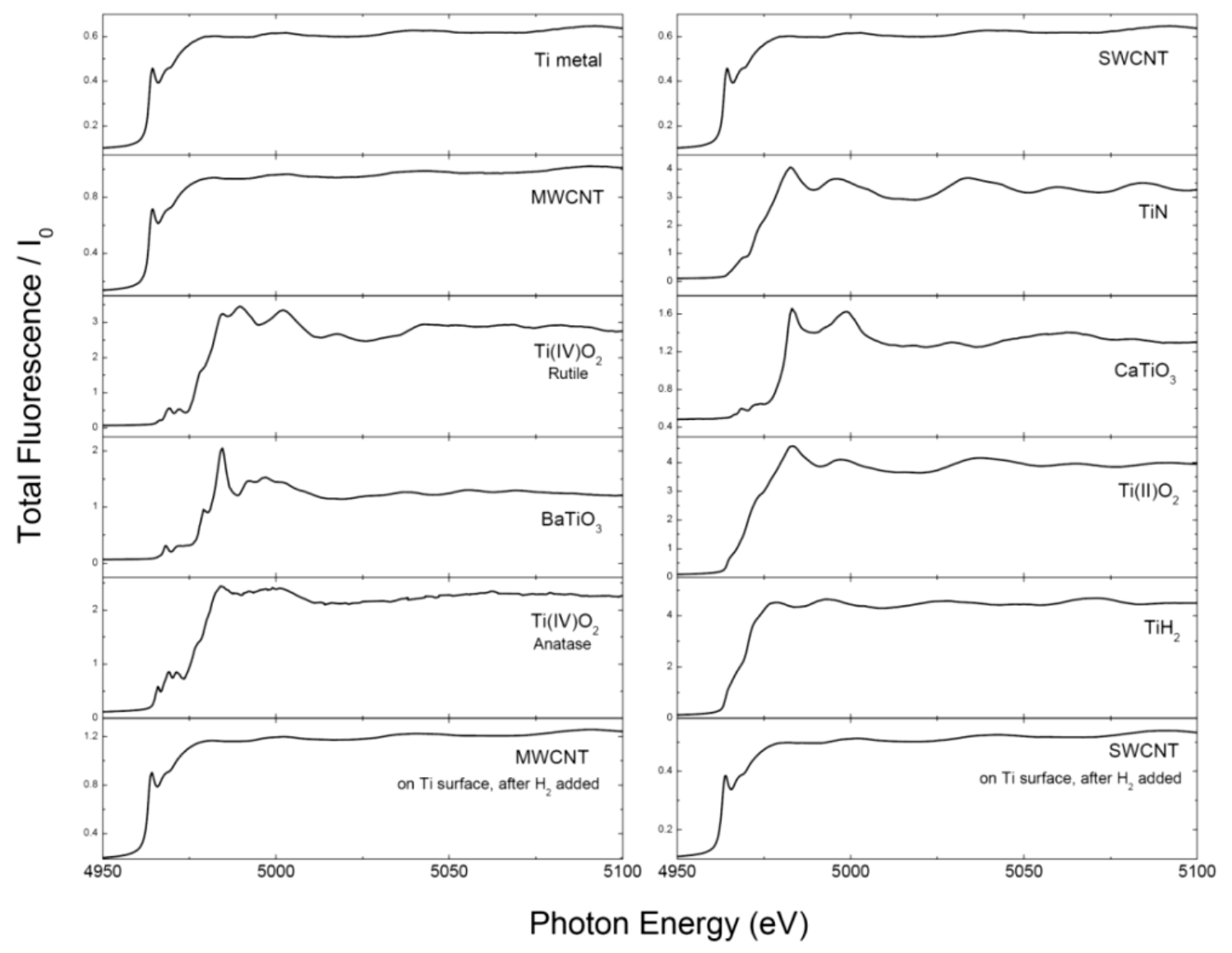

Figure 4. X-ray absorption spectra taken near the Ti $K$-edge for single and multi-walled carbon nano tubes, and reference sample materials.
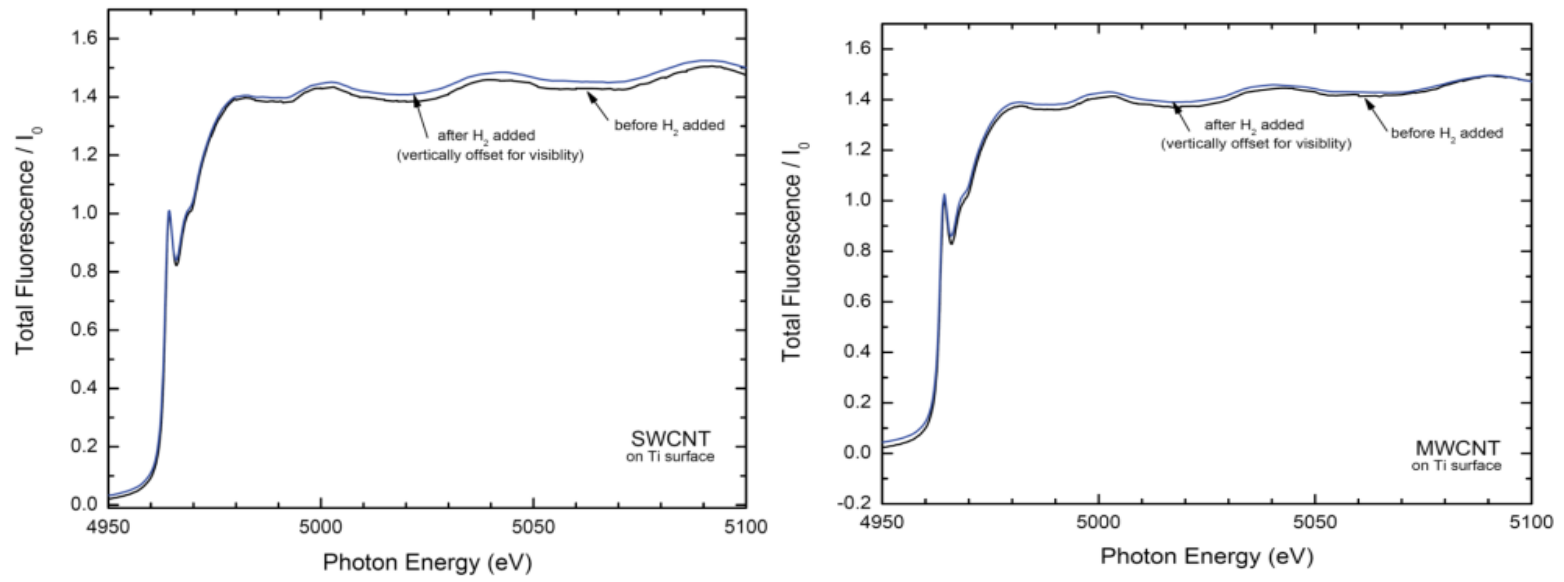

Figure 5. X-ray absorption spectra taken near the Ti $K$-edge of single and multi-walled carbon nano tubes on a titanium surface, taken before and after $\mathrm{H}_{2}$ loading. This indicated that carbon nano tubes were practically invisible to the method, and virtually no change was observed with or without $\mathrm{H}_{2}$ loading. 


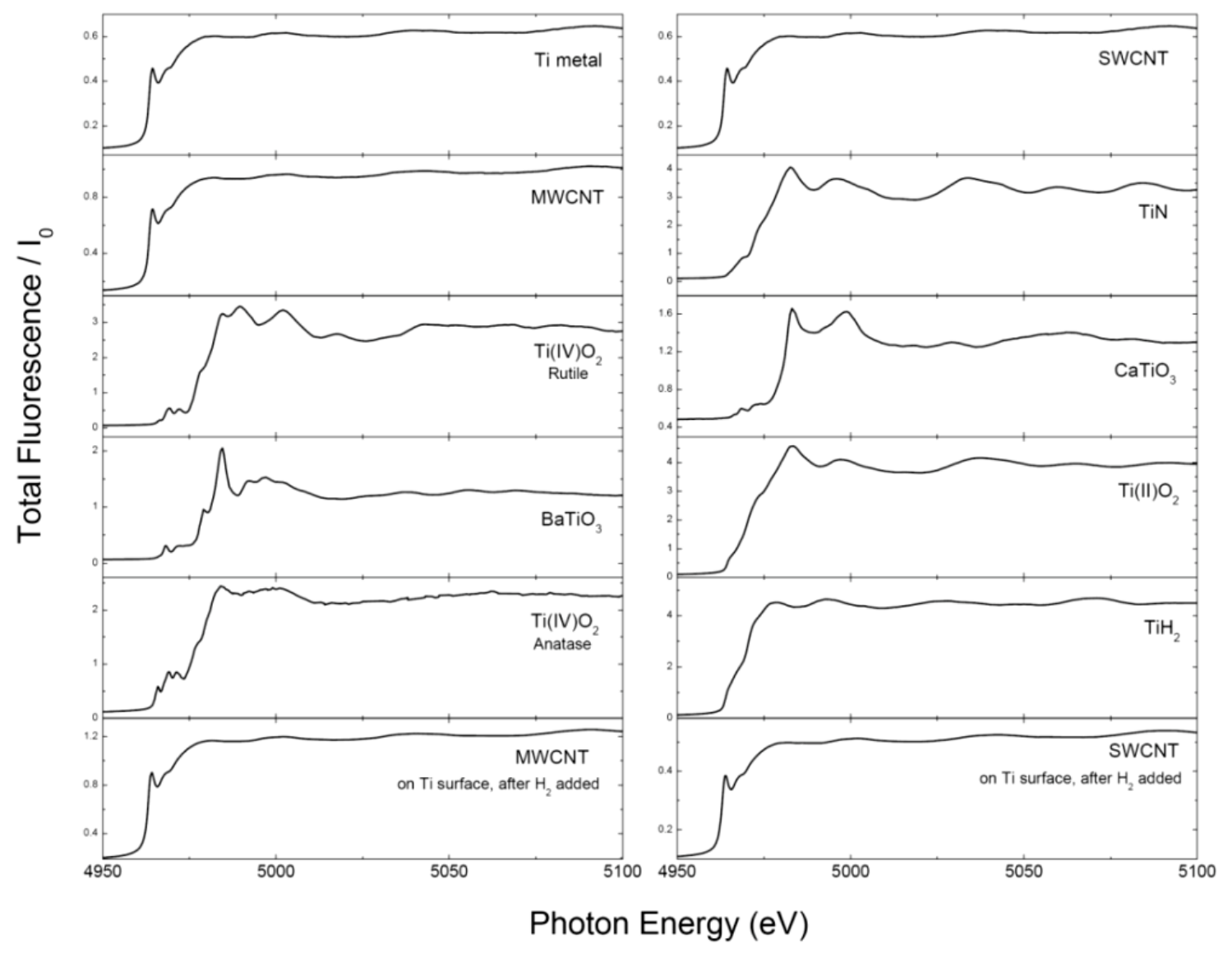

Figure 4. X-ray absorption spectra taken near the Ti $K$-edge for single and multi-walled carbon nano tubes, and reference sample materials.
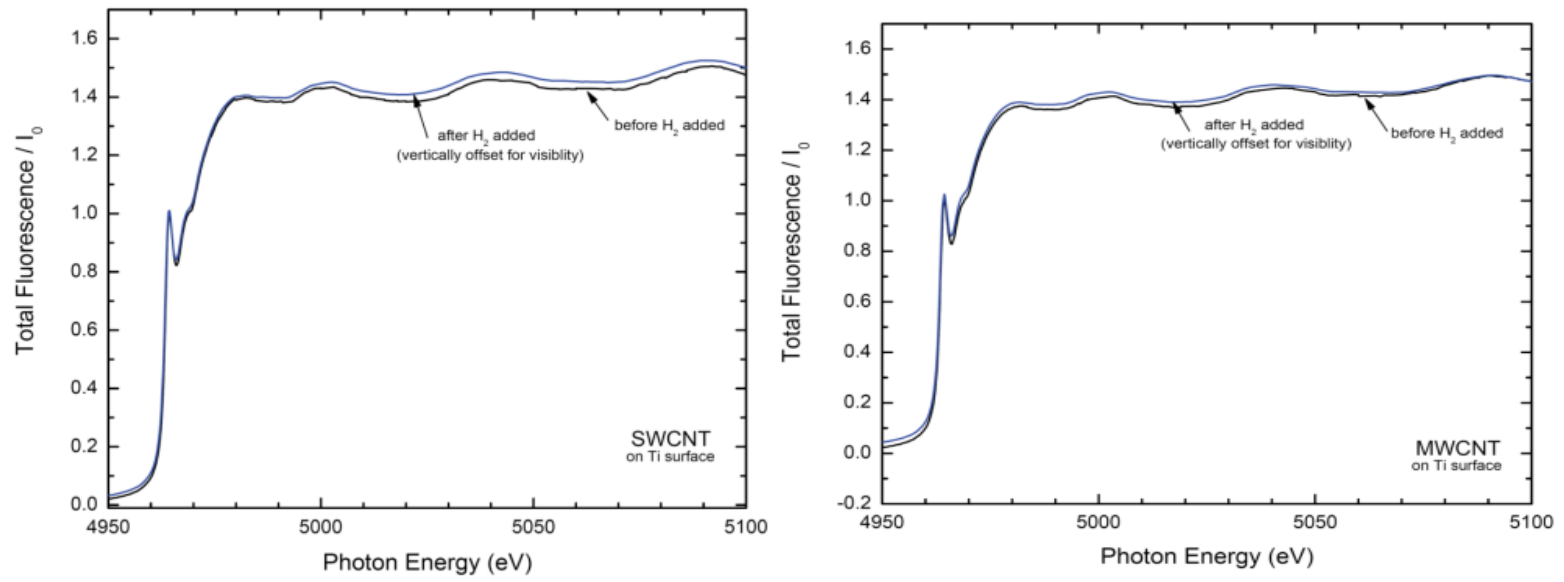

Figure 5. X-ray absorption spectra taken near the Ti $K$-edge of single and multi-walled carbon nano tubes on a titanium surface, taken before and after $\mathrm{H}_{2}$ loading. This indicated that carbon nano tubes were practically invisible to the method, and virtually no change was observed with or without $\mathrm{H}_{2}$ loading. 


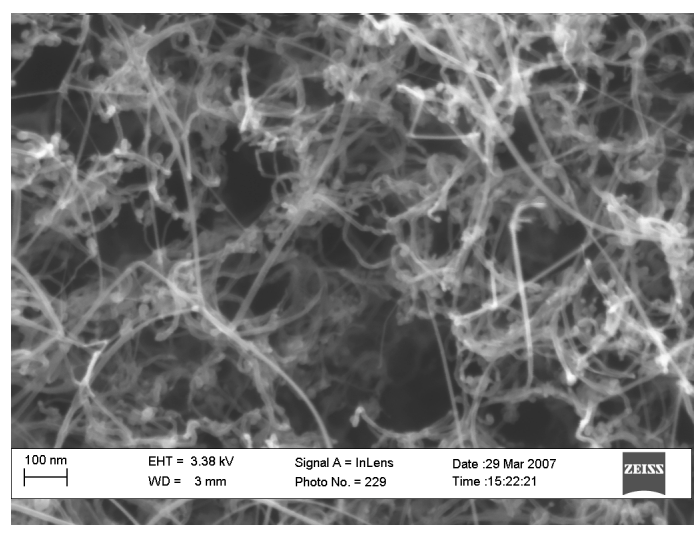

Figure 6. One of the many images taken of single walled carbon nano-tubes on a Ti substrate. Image was taken at the Molecular Foundry using a Zeiss electron microscope, during the October 2006 shutdown of the Advanced Light Source.

Follow up measurements were to be performed on nano-tube materials containing a titanium cap closing the nano-tubes manufactured on both silicon and titanium substrates, unfortunately FCast funding for this sub-task was cancelled before the experiments could be performed. This material was to be created at the University of California Berkeley.

The construction of equipment vital to this experimental proposal and the external collaborations it generated has allowed us to quickly move to the investigation of cadmium based solar cell materials.

- $\quad$ Problems encountered departure from planned methodology and an assessment of their impact on the project results.

1. After the upgrades and construction of the necessary equipment required to perform the proposed experiments had been completed, we encountered extreme difficulties obtaining the necessary carbon nano tube materials. Initially they were to be created by other members as part of the project; unfortunately we were unable to obtain the necessary materials from this source. A delay of approximately 6 months was caused as we searched for groups which could create the nano tube / Ti materials we required. The materials were finally obtained by collaboration with the Materials Science and Technology Division of Lawrence Livermore National Laboratory and some reference materials were provided by LBNL's Center for X-ray Optics.

2. Synchrotron radiation at the Advanced Light Source of Lawrence Berkeley National Laboratory was unavailable during the period October 2006 to January 2007. The impact on the project was a delay in instrumentation testing and actual experiments.

\section{Identify products developed under the award and technology transfer activities.}

a. Publications (list journal name, volume, issue), conference papers, or other public releases of results).

- Poster presented at UNLV Renewable Energy Symposium, Las Vegas Aug 2007: 
"Synchrotron X-Ray Spectroscopy Studies for $\mathrm{H}_{2}$ Storage" by Oliver Hemmers, Kristina Lipinska-Kalita, Dennis Lindle, Iraida Demchenko and Wayne Stolte.

- Poster presented at Users' Association Annual Meeting, Berkeley, CA, 4-6 October 2007, "Synchrotron X-ray spectroscopy studies for $\mathrm{H}_{2}$ storage" by O.Hemmers, $\mathrm{K}$ Lipinska-Kalita, D.W. Lindle I. Demchenko, and W.C. Stolte,.

- Poster presented at ALS Users' Association Annual Meeting, Berkeley, CA, 4-6 October 2007, "Transmission versus fluorescence mode collection in XAS: Ti K-edge of TiN" by I.N. Demchenko, W.C. Stolte, N. Birknern, R. Minikayev, A. Wongjamras, and M. Chernyshova.

b. Web site or other Internet sites that reflect the results of this project;

- None

c. Networks or collaborations fostered;

- We have initiated a collaboration with the Materials Science and Technology Division of Lawrence Livermore National Laboratory, that specializes in fabrication and deposition of CNTs on different substrates.

- A collaboration with the Center for X-Ray Optics (CXRO) at Lawrence Berkeley National Laboratory was initiated

- A collaboration with the Molecular Foundry at Lawrence Berkeley National Laboratory was also initiated

d. Technologies/Techniques;

- An X-Ray Emission Setup was modified for use with solid samples and a new switchable crystal bender was installed to allow the measurement of higher photon energies at beamline 9.3.1 at the Advanced Light Source of Lawrence Berkeley National Laboratory.

- An X-Ray Absorption Setup was modified at beamline 9.3.1 the Advanced Light Source of Lawrence Berkeley National Laboratory.

e. Inventions/Patent Applications, licensing agreements; and

- None

f. Other products, such as data or databases, physical collections, audio or video, software or netware, models, educational aid or curricula, instruments or equipment.

- None 


\section{ATTACHMENT C}

Task 2: Metal Hydrides

PI: A. Cornelius

We were active in publishing our results studying the hydrogen bonding in metal hydrides by determining the equation of state by $P-V$ measurements; namely on $\mathrm{NaBH}_{4}$ and related materials. The results have been presented at 8 conferences and 8 manuscripts have appeared in peer-reviewed journals (listed below). Some of this work has been supported with theoretical work in collaboration with PI Eunja Kim.

Moving beyond $\mathrm{NaBH}_{4}$, we determined the high-pressure crystal structure of Li3AlH6 and $\mathrm{KBH}_{4}$ and $\mathrm{RbBH}_{4}$. The reasons for this are twofold. First we aim to understand the high pressure transitions predicted so that we can validate the theoretical models explaining the phase transition sequence. Second, we believe these studies are important for developing novel mixed hydride systems with suitable catalysts, for which the structural information will provide new insights. Hence, we have performed highpressure x-ray diffraction experiments up to $27 \mathrm{GPa}$ and high temperature $\mathrm{x}$-ray diffraction up to $110^{\circ} \mathrm{C}$. We have also worked with Changfeng Chen and Eunja Kim who have contributed theoretical calculations.

\section{Final List of Publications}

1. "Structural phase transitions in the potential hydrogen storage compound $\mathrm{RbBH}_{4}$ under compression.” Ravhi S. Kumar and Andrew L. Cornelius, J.Alloys and Comp. 476, 5 (2008)

2. "Effect of Pressure and Temperature on Structural Stability of Potential Hydrogen Storage Compound $\mathrm{Li}_{3} \mathrm{AlH}_{6}$.” Ravhi S. Kumar, Xuezhi Ke, Andrew L. Cornelius, and Changfeng Chen, Chem. Phys. Lett. 460, 442 (2008).

3. "In situ observations of temperature- and pressure-induced phase transitions in $\mathrm{TiH}_{2}$ : Angle-dispersive and synchrotron energy-dispersive X-ray diffraction studies.” P.E. Kalita, A.L. Cornelius, K.E. Lipinska-Kalita, Cedric L. Gobin, H. Peter Liermann, J. Phys. Chem. Solid 69, 2240 (2008).

4. "Structural Phase Transitions in the Potential Hydrogen Storage Compound $\mathrm{KBH}_{4}$ under Compression.” R. Kumar, E. Kim, and A.L. Cornelius, J. Phys. Chem. C 112 (2008) 8452.

5. "Pressure-driven phase transitions in $\mathrm{NaBH}_{4}$ : Theory and experiments.” E. Kim, R. Kumar, P.F. Weck, A.L. Cornelius, M. Nicol, S.C. Vogel, J. Zhang, M. Hartl, L. Daemen, and Y. Zhao, J. Phys. Chem. B 111, 11873 (2007).

6. "Pressure-induced structural phase transition in $\mathrm{NaAlH}_{4}$." Ravhi S. Kumar, Eunja Kim, Oliver Tschauner, Andrew L. Cornelius, Martin P. Sulic, and Craig M. Jensen, Phys. Rev. B 75, 174110 (2007).

7. "Bonding changes in single wall carbon nanotubes (SWCNT) on $\mathrm{Ti}$ and $\mathrm{TiH}_{2}$ addition probed by X-ray Raman scattering.” R.S. Kumar, A.L. Cornelius, M.G. Pravica, M.F. Nicol, M.Y. Hu, and P.C. Chow, Diamond and Related Mater. 16, 1136 (2007).

8. “Structural Transitions in $\mathrm{NaBH}_{4}$ Under Pressure.” Ravhi S. Kumar and A.L. Cornelius, Appl. Phys. Lett. 87, 261916 (2005). 


\section{ATTACHMENT D}

Task 3: Synthesize and characterize mesoporous conductive polymer/Pd structures with subsequent evaluation of hydrogen storage performance

PI: David W. Hatchett, 702-895-3509, david.hatchett@unlv.edu,

Project Objective: The objective of this task is to prepare conductive polymer/Pd structures with subsequent evaluation of hydrogen storage performance.

Background: The uptake and storage of hydrogen in conductive polymers is fundamentally interesting due to the high intrinsic electron density and ability to dope the materials with protons, anions, and metal species. Previous studies with conductive polymer systems have been contradictory. In one case the uptake of $7-8 \%$ hydrogen is reported. In another report thepolymers had no ability to store hydrogen. Questions remain regarding the ability of the material to store hydrogen. Additional issues including oxidation state and proton doping must be considered also. The modification of the polymer with Pd metal was proposed to enhance the storage capabilities of the material. There is conflicting literature regarding the incorporation of Pd into polyaniline. The methods used to incorporate the Pd species can be defined by the method of Pd incorporation.

1. Chemical synthesis using either Pd(iv) or Pd(ii) and the monomer/oligomer.

2. Electrochemical synthesis of PANI followed by reduction of Pd(iv) or Pd(ii).

Each method produces different materials with varying composition of Pd. The last year has been focused on evaluating the hydrogen storage properties of the materials prepared. We have obtained independent measurement of the hydrogen sorption of the most promising material and have provided this data for comparison. This report serves as the final annual report for work performed on this project.

Status: We have synthesized the polymer chemically and electrochemically incorporating Pd(ii) and Pd(iv) species in both materials. We have a total of twelve different materials produced using electrochemical and chemical synthesis. Contrary to many materials produced, these materials do not require liquid nitrogen temperatures to observe hydrogen sorption. We have evaluated the reproducibility of the sorption/desorption of hydrogen for all of the materials produced and have identified the two most promising PANI/Pd composites. Hydrogen sorption data is presented in Figure 1. The unsorbed hydrogen elutes in less than one minute with the sorbed hydrogen elution initiated at $~ 3.5$ minutes. The elution of sorbed 
hydrogen indicating fast adsorption kinetics. The adsorption/desorption for PANI/Pd composite materials as a function of desorption temperature and exposure time is presented in Figure 2. The data indicates that the desorption temperature influences the desorption of hydrogen from the composites materials. The percent hydrogen measured at $200{ }^{\circ} \mathrm{C}$ reached a maximum value of $1.7 \%$ by weight. For comparison a value of $0.5 \%$ is obtained for the same composite material at a desorption temperature of $160^{\circ} \mathrm{C}$.
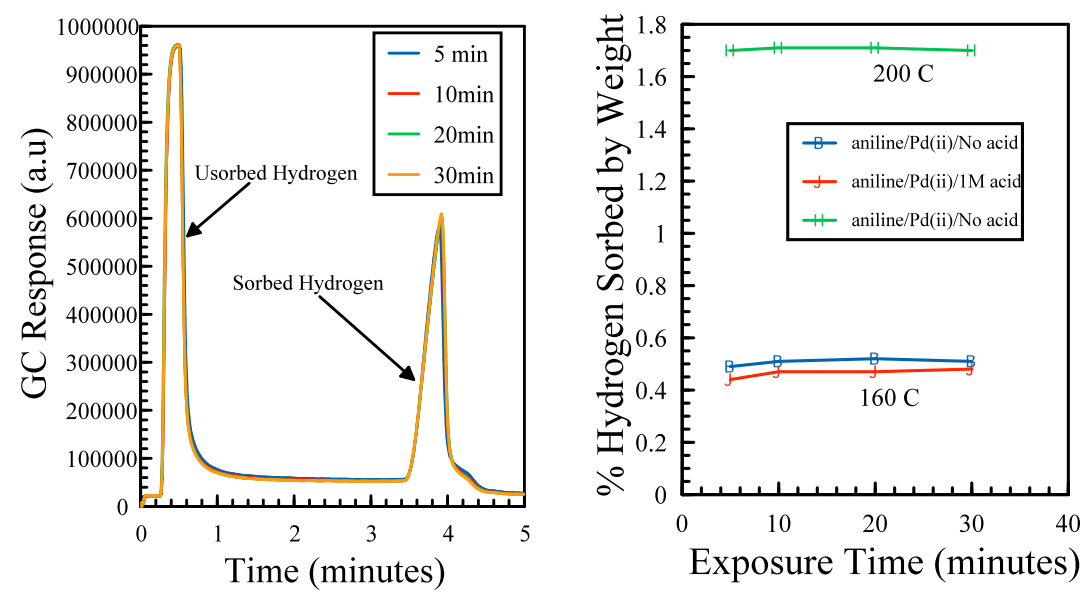

Figure 1. Left: Hydrogen sorption at Aniline/Pd(ii) composite. Right: Percent hydrogen sorption at Aniline/Pd(ii) composite as a function of exposure time at desorption temperatures of $200{ }^{\circ} \mathrm{C}$ and $160{ }^{\circ} \mathrm{C}$.

For comparison the sorption properties of the Aniline/Pd(ii) composite was independently measured at the Southwest Research Institute (SWRI). The institute is designated as the independent measurement facility for hydrogen sorption by the Department of Energy. The results of their measurements are presented in Figure 2.

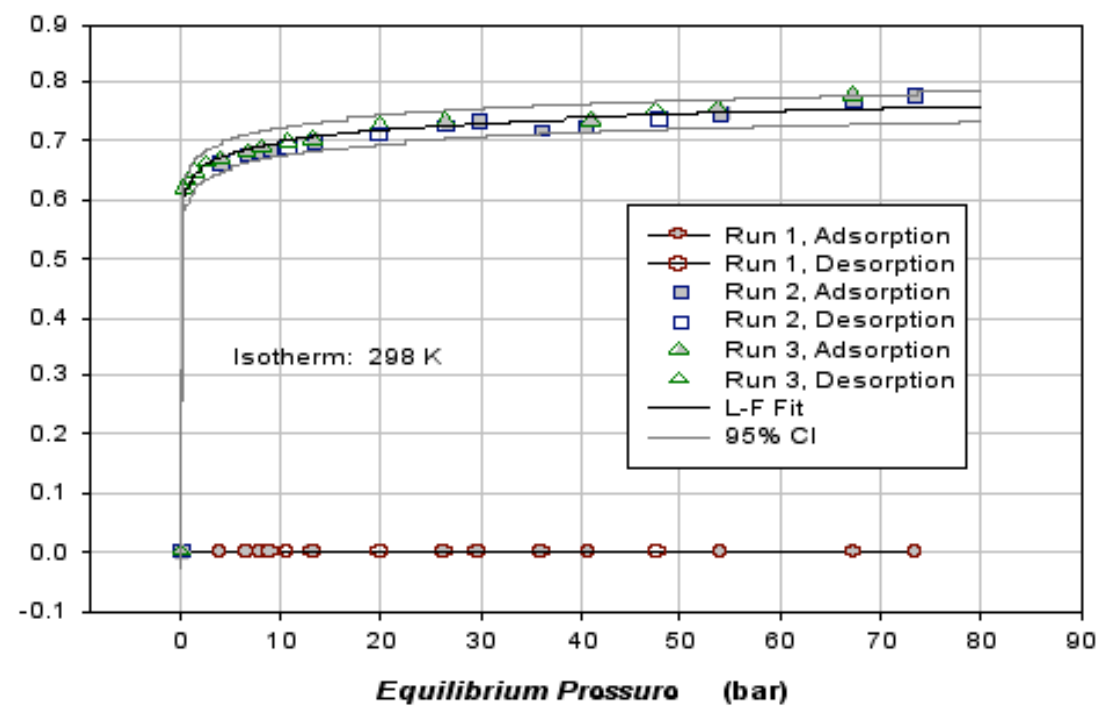

Figure 2. Hydrogen sorption/desorption isotherms for Aniline/Pd(ii) composite measure independently by Southwest Research Institute. 
The data from Figure 2 shows maximum hydrogen sorption of $0.8 \%$ for the composite held at room temperature, as a function of equilibrium pressure. The desorption temperature was 175 ${ }^{\circ} \mathrm{C}$. The temperature is an intermediate temperature compared to Figure 1 and the percent hydrogen measured is in between those values measured in our lab. However, the results verify the sorption values obtained in our laboratory for the composite material. More importantly, the percent hydrogen sorption observed for the composite is high for materials at room temperature based on the literature to date. The data obtained from these studies shows interesting behavior. The composite material provided for independent testing initially showed no hydrogen sorption on the first trial. However in each subsequent trial the sample showed reproducible hydrogen sorption. This implies that the material undergoes a conditioning during the first trial. In addition the adsorption/desorption isotherms for the trials showing hydrogen sorption do not have any appreciable hysteresis indicating the adsorption/desorption is reversible.

Conclusions: Our goals for identifying and evaluating the hydrogen sorption for the PANI/Pd composites were achieved. From the experimental results we have developed a method for converting $\mathrm{Pd} /$ aniline complexes into polymer with $\mathrm{Pd}(0)$ with demonstrated hydrogen sorption properties. We evaluated the sorption using a simple prototype for hydrogen sorption device produced in-house. Finally we had the hydrogen sorption properties of our most promising composite independently verified. 


\section{ATTACHMENT E}

Project Title: Fuel Cells and Storage Technology, Tasks 1, 4, 5

PI: Balakrishnan Naduvalath

The project supported two postdoctoral scholars (Dr. Philippe Weck and Dr. Dhilip Kumar), one Assistant Research Professor (Dr. Tarakeshwar Pilarisetty) and a visiting graduate student from China (Chengang Zhou) who co-authored three papers in 2007/2008. Dr. Weck is currently part of the Radiochemistry program at UNLV and he is leading the computational aspect of the Radiochemistry program. Dr. Dhilip Kumar took up a postdoc position at University of Michigan, Ann Arbor in Dec. 2008. Dr. Pilarisetty moved to Arizona State University in January 2010 as an Assistant Research Professor. Chenggang returned to China in 2007 and took up a teaching position after completing his $\mathrm{PhD}$ work.

The project led to 17 peer-reviewed publications [1-17]. The primary focus of our studies has been on fundamental understanding of the interaction between hydrogen and different nanomaterials. Since this has relevance to both hydrogen storage and heterogeneous catalysis, we explored issues related to both topics.

\section{Task 1 \&4: Hydrogen storage and fuel cell membranes:}

On hydrogen storage, we examined the efficacy of model organometallic compounds consisting of $\mathrm{Sc}, \mathrm{Ti}$, and $\mathrm{V}$ transition metal atoms bound to $\mathrm{CmHm}$ rings $(\mathrm{m}=4-6)$ using density functional theory (DFT). This work was published in J. Chem. Phys. (2007) and it has so far been cited 22 times, including Physical Review Letters and Applied Physics Letters. In a related Work Performed in collaborations with Prof. Boris Yakobson at Rice University and Dr. Hansong Cheng at Airproducts and Chemicals, Inc., we proposed a novel class of 3-D materials consisting of ultra-small diameter single-walled carbon nanotubes covalently functionalized by organic ligands as potential hydrogen storage media. Ab initio Car-Parrinello molecular dynamics (MD) simulations showed that the proposed nanoframeworks are energetically and thermodynamically stable up to at least $600 \mathrm{~K}$. The proposed structure, published in Chem. Phys. Lett. (2007), was experimentally validated in a recent work published by Tour and coworkers at Rice university and NREL (Leonard et al., J. Am. Chem. Soc. 131, 723 (2009)). They demonstrated the utility of similar nanoengineered carbon scaffolds for hydrogen storage. Based on initial measurements, nanoscaffolds with surface areas of $2000 \mathrm{~m}^{2} / \mathrm{g}$ are capable of a hydrogen uptake of $7.4 \mathrm{wt} \%$ at 2 bar. In a related study we investigated the successive adsorption of hydrogen atoms on boron nitride graphitic sheets. Our work showed that hydrogen adsorption on the BN surfaces is stabilized by the formation of contiguous H-H orthodimer structures. This work was featured on the cover of Phys. Chem. Chem. Phys. \{Phys. Chem. Chem. Phys., 10, 5184-5187 (2008)\}. In a collaborative work with FCAST team member and organic chemistry colleague Dr. Chulsung $\mathrm{Bae}$, we investigated the mechanism of proton transport in new polymer-electrolyte membranes based on sulfonated aromatic polyarylenes synthesized in Dr. Bae's group for high temperature fuel cell applications. This work (purely computational) was featured on the cover of J. Phys. Chem. B. \{J. Phys. Chem. B 112, 3283-3286 (2008)\}.

Task 5: Nanocatalysts and clusters: During the course of our work on hydrogen adsorption on titanium decorated organic systems, we realized that there has not been any 
detailed study of electronic structures and reactivity of small titanium clusters. This led us to undertake an extensive investigation of the electronic structures and stability of small Ti clusters. The sequential growth of titanium clusters up to 15 atoms, and for the first time, the dissociative chemisorption of $\mathrm{H}_{2}$ on the corresponding minimum energy clusters were investigated using DFT. Results of these studies were published in J. Phys. Chem. C (2007). The studies showed a strong correlation between the chemisorption energy and cluster size.

In realistic modeling of heterogeneous catalysis involving nanoparticles, one has to take into account the changes in the electronic structure of the catalyst particles due to the interaction with multiple adsorbate molecules. Theoretical studies of chemisorption are generally limited to a single adsorbate molecule interacting with the metal surface or the cluster. To model more realistic catalytic and hydrogen storage systems containing titanium, we explored the effect of hydrogen saturation on the electronic structures of small $\mathrm{Ti}$ clusters. Calculations on $\mathrm{Ti}_{4}, \mathrm{Ti}_{7}$, $\mathrm{Ti}_{13}$, and the larger $\mathrm{Ti}_{55}$ cluster revealed that at high hydrogen concentrations, hydrogen multicenter bonds become prevalent. A key result of our study is that the hydrogen multicenter bonds are characterized by vibrational frequencies in the $1100-1500 \mathrm{~cm}^{-1}$ regime, and that the adsorbed hydrogen could be easily released by selective excitation of vibrational modes. Due its high stability, much of our studies focused on different conformers of $\mathrm{Ti}_{13}$, but we also investigated hydrogen saturation $\mathrm{Sc}_{13}$ and $\mathrm{Zr}_{13}$ clusters. These studies led to a series of papers: J. Phys. Chem. A (2008), J. Chem. Phys. (2008, 2009), and Phys. Rev. B (2009). In a parallel work, in collaboration with Dr. Hansong Cheng, we investigated electronic structures of small clusters of Pd and Pt as well as Pt-Co alloy systems to explore how alloying and doping affect reactivity of small transition metal clusters. We also looked into the growth of small Pt clusters on $\alpha-\mathrm{Al}_{2} \mathrm{O}_{3}$ surface and investigated the nature of cluster-support interaction. Though electronic structures of subnanoclusters of Pt and Pd have been extensively investigated their interaction with support materials has not been well understood. The works on Pd clusters and growth of Pt clusters on $\mathrm{Al}_{2} \mathrm{O}_{3}$ support were published in 2007. The work on $\mathrm{H}_{2}, \mathrm{O}_{2}$, and $\mathrm{CO}$ chemisorption on $\mathrm{Pt}_{4}$ and $\mathrm{Pt}_{3} \mathrm{Co}$ clusters was published in J. Chem. Phys. in 2008.

\section{Publications}

1. P. F. Weck, N. Balakrishnan, J. Brandao, C. Rosa, and W. Wang, Dynamics of the $\mathrm{O}\left({ }_{3} \mathrm{P}\right)+\mathrm{H}_{2}$ reaction at low temperatures: Comparison of quasiclassical trajectory with quantum scattering calculations, J. Chem. Phys. 124, 074308 (2006).

2. T.G. Lee, N. Balakrishnan, R. C. Forrey, P. C. Stancil, D. R. Schultz, and G. J. Ferland, State-to-state rotational transitions in $\mathrm{H}_{2}+\mathrm{H}_{2}$ collisions at low temperatures, J. Chem. Phys. 125,114302 (2006).

3. P. F. Weck, E. Kim, N. Balakrishnan, F. Poineau, C. B. Yeamans, and K. Czerwinski, "First principles study of single-crystal uranium mono-and dinitride", Chem. Phys. Lett. 443, 82-86 (2007).

4. C. Zhou, J. Wu, T. J. Dhilip Kumar, N. Balakrishnan, R. C. Forrey, and H. Cheng, "The Growth Pathway of Pt Clusters on $\alpha$-Al2O3 (0001) Surface", J. Phys. Chem. C 111, 1378613793 (2007).

5. P. F. Weck, E. Kim, N. Balakrishnan, H. Cheng, and B. I. Yakobson, "Designing carbon nanoframeworks tailored for hydrogen storage", Chem. Phys. Lett. 439, 354-359 (2007). 
6. T. J. Dhilip Kumar, P. F. Weck and N. Balakrishnan, "Evolution of small Ti clusters and the dissociative chemisorption of H2 on Ti”, J. Phys. Chem. C 111, 7494-7500 (2007).

7. P. F. Weck, T. J. Dhilip Kumar, E. Kim, and N. Balakrishnan, "Computational study of hydrogen storage in organometallic compounds”, J. Chem. Phys. 126, 094703 (2007).

8. C. Zhou, C. Luo, J. Wu, T. J. Dhilip Kumar, N. Balakrishnan, R. C. Forrey, and H. Hansong, "First principles study of small palladium cluster growth and isomerization", Int. J. Quant. Chem. 107, 1632-1641 (2007).

9. P. F. Weck, E. Kim, S. S. Lepp, N. Balakrishnan, and H. R. Sadeghpour, "Dimerinduced stabilization of $\mathrm{H}$ adsorbate cluster on BN(0001) surface", Phys. Chem. Chem. Phys., 10, 5184-5187 (2008) [Cover article, issue 34, 2008].

10. T. J. Dhilip Kumar, P. Tarakeshwar, and N. Balakrishnan, "Structural, energetic and electronic properties of hydrogenated titanium clusters", J. Chem. Phys. 128, 194714 (2008).

11. T. J. Dhilip Kumar, C. Zhou, H. Cheng, R. C. Forrey, and N. Balakrishnan, "Effect of Co doping on the catalytic activity of small Pt clusters", J. Chem. Phys. 128, 124704 (2008).

12. E. Kim, P. F. Weck, N. Balakrishnan, and C. Bae, "Nanoscale building blocks for the development of novel proton-exchange membrane for fuel cells", J. Phys. Chem. B 112, 3283-3286 (2008) (Cover article, March 20, 2008 issue).

13. P. Tarakeshwar, T. J. Dhilip Kumar, and N. Balakrishnan, "Nature of hydrogen interaction and saturation on small Titanium clusters", J. Phys. Chem. C 112, 2846-2854 (2008).

14. P. Tarakeshwar, T. J. Dhilip Kumar, and N. Balakrishnan, "Hydrogen multicenter bonds and reversible hydrogen storage”, J. Chem. Phys. 130, 114301 (2009).

15. T. J. Dhilip Kumar, P. Tarakeshwar, and N. Balakrishnan, "Geometric and Electronic Structures of Hydrogenated Transition Metal ( $\mathrm{M}=\mathrm{Sc}, \mathrm{Ti}, \mathrm{Zr})$ clusters”, Phys. Rev. B 79, 205415 (2009).

16. P. Tarakeshwar and N. Balakrishnan, "Low-cost alloy clusters for nanocatalysis", (manuscript in preparation).

17. H. Mai, P. Tarakeshwar and N. Balakrishnan, "Charge and Adsorbate Saturation Effects on the Energies, Geometries, and Transition States of Doped Platinum Clusters", (manuscript in preparation).

\section{Presentations}

- Balakrishnan Naduvalath, Molecular adsorption and dissociation on transition metal clusters and alloys, presentation to representatives from United Technology Corporation, 03/28/07, UNLV.

- T. J. Dhilip Kumar, P. F. Weck, and Balakrishnan Naduvalath, Evolution of small Ti clusters and the dissociative chemisorption of $\mathrm{H}_{2}$ on Ti, APS March Meeting, March 5-9, 2007, Denver, CO. 
- T. J. Dhilip Kumar, C. Zhou, and Balakrishnan Naduvalath, First principles study of adsorption and dissociation of $\mathrm{H}_{2}, \mathrm{O}_{2}$, and $\mathrm{CO}$ on $\left\langle-\mathrm{Al}_{2} \mathrm{O}_{3}\right.$ (0001) supported Pt-Co alloy, APS March Meeting, March 5-9, 2007, Denver, CO.

- P. F. Weck, E. Kim, N. Balakrishnan, and H. Cheng, Design of tailored carbon nanostructures for hydrogen storage, APS March Meeting, March 5-9, 2007, Denver, CO.

- T. J. Dhilip Kumar, C. Zhou, and Balakrishnan Naduvalath, R. C. Forrey and H. Cheng, First principles study of adsorption and dissociation of $\mathrm{H}_{2}, \mathrm{O}_{2}$, and $\mathrm{CO}$ on $\mathrm{Pt}_{4}$ and $\mathrm{Pt}_{3} \mathrm{Co}$ clusters, DAMOP Meeting, June 5-9, 2007, Calgary, Canada.

- Balakrishnan Naduvalath, Hydrogen storage and catalysis, Presentation to Jesse Adams, DOE program Manager, during his site visit to UNLV on 03/14/08.

- P. Tarakeshwar, T. J. Dhilip Kumar, and N. Balakrishnan, Hydrogen Multicenter Bonds on Small Metal Clusters, APS March Meeting, March 10-14, 2008, New Orleans, Louisiana.

- T. J. Dhilip Kumar, P. Tarakeshwar, and N. Balakrishnan, Sequential Dissociative Chemisorption of $\mathrm{H}_{2}$ on Ti13 Cluster, APS March Meeting, March 10-14, 2008, New Orleans, Louisiana.

- P. F. Weck, E. Kim, C. Bae, and N. Balakrishnan Nanoscale building blocks for the development of novel proton-exchange membranes fuel cells: A first-principles study, APS March Meeting, March 10-14, 2008, New Orleans, Louisiana.

- Euja Kim, Philippe Weck, Balakrishnan Naduvalath, Hansong Cheng, Boris Yakobson, First-Principles Study of Carbon Nanoframeworks Tailored for Hydrogen Storage, Hydrogen Multicenter Bonds on Small Metal Clusters, APS March Meeting, March 10-14, 2008, New Orleans, Louisiana.

- P. F. Weck, E. Kim, N. Balakrishnan, H. Cheng, and B. Yakobson, Lithium-coated carbon nanoframeworks tailored for hydrogen storage, International symposium on material issues in a hydrogen economy, November 12-15, 2007, Richmond, Virginia.

- Balakrishnan Naduvalath, Fundamental studies of hydrogen storage and catalysis, UNLV Energy Symposium, August 16, 2007, UNLV.

- Tarakeshwar Pilarisetty, Dhilip Kumar Thogluva Janardhanan, and N. Balakrishnan, Hydrogen storage and catalytic properties of transition metal clusters: Bonding changes and saturation effects, 236th American Chemical Society National Meeting, Philadelphia, PA, August 17-21, 2008.

- T. J. Dhilip Kumar, P. Tarakeshwar, and N. Balakrishnan, Geometric and Electronic Structures of Ti-Al and Hydrogenated Ti-Al Nanoclusters, American Chemical Society Western Regional Meeting, Las Vegas, NV, September 24-28, 2008.

- T. J. Dhilip Kumar, P. Tarakeshwar, and N. Balakrishnan, The Effect of Alloying on CO Tolerance of Pt Nanoparticles, American Chemical Society Western Regional Meeting, Las Vegas, NV, September 24-28, 2008.

- Huy Mai, T. J. Dhilip Kumar, P. Tarakeshwar, and N. Balakrishnan, Computational Studies of $\mathrm{H}_{2}, \mathrm{O}_{2}$, and $\mathrm{CO}$ Adsorption on Pt and Pt-Ru Catalyst Clusters for Hydrogen Fuel Cell Applications, American Chemical Society Western Regional Meeting, Las Vegas, NV, September 24-28, 2008. 


\section{Grant proposals submitted:}

- Surface coverage and catalytic properties of metal nanoclusters and nanoparticles, Balakrishnan Naduvalath (PI), Tarakeshwar Pilarisetty (co-PI), submitted to National Science Foundation, 07/01/08-06/30/11, \$389,309 (declined).

- Hydrogen multicenter bonds and hydrogen storage, Balakrishnan Naduvalath (PI), Tarakeshwar Pilarisetty (co-PI), submitted to DOE BES, 07/01/08-06/30/11, \$435,384 (declined).

- Unraveling the role of adsorbate-metal interactions on the catalytic properties of transition metal clusters, Tarakeshwar Pilarisetty (PI), Balakrishnan Naduvalath (co-PI), submitted to American Chemical Society Petroleum Research Fund, 09/01/08-08/31/10, $\$ 89,750$ (declined).

- Effects adsorbate saturation and external fields on the catalytic activity of metal nanoclusters Balakrishnan Naduvalath (PI), Tarakeshwar Pilarisetty (co-PI), submitted to DOE BES, 07/01/09-06/30/12, \$272,786 (declined).

- Surface coverage and catalytic properties of titanium and aluminum containing nanoclusters and nanoparticles, Balakrishnan Naduvalath (PI), Tarakeshwar Pilarisetty (co-PI), submitted to National Science Foundation, 07/01/09-06/30/12, \$289,219 (declined).

- Collective effects of adsorbed and co-adsorbed molecules on the kinetics and reactivity of metal nanoclusters, Balakrishnan Naduvalath (PI), Tarakeshwar Pilarisetty (co-PI), submitted to National Science Foundation, 07/01/10-06/30/13, \$300,103 (pending). 


\section{ATTACHMENT F}

\section{Task 4 (co-PI: Chulsung Bae)}

\section{Objectives}

- Develop new hydrocarbon-based polymer electrolyte membranes that can cost less and perform better than Nafion especially at high temperature $\left(120^{\circ} \mathrm{C}\right)$ and low humidity

\section{Technical Barriers}

This project addresses the following technical barriers from the Fuel Cell section of the Hydrogen, Fuel Cells and Infrastructure Technologies Program Multi-Year Research, Development and Demonstration Plan:

(A) Durability of Polymer Electrolyte Membrane (PEM)

(B) Costs of PEM

(C) Performance of PEM

\section{Technical Targets}

This project aims to develop of new sulfonated fuel cell membranes that can outperform the state-of-the-art membrane, Nafion, at high temperature $\left(120^{\circ} \mathrm{C}\right)$ and low relative humidity. The successful development of such membranes will address the following DOE 2010 technical targets.

- Membrane conductivity: $0.1 \mathrm{~S} / \mathrm{cm}$ (at $120^{\circ} \mathrm{C}$ ), $0.07 \mathrm{~S} / \mathrm{cm}$ (at room temperature)

\section{Accomplishments}

- Developed new sulfonated hydrocarbon-based PEMs that perform better than Nafion in proton conductivity and have acceptable water uptake

\section{Introduction}

Most ionomer materials currently being tested in polymer electrolyte membrane fuel cells demonstration units are based on perfluorosulfonic acid polymers, such as Nafion. However, shortcomings of these materials seriously limit wide application of fuel cells as power sources. The objective of this project is to develop new hydrocarbon-based high-performance sulfonated PEMs that can be manufactured at low cost.

\section{Approach}

Aromatic polyamides are known to have excellent chemical stability and mechanical properties that are critical features for an effective polymer electrolyte membrane (PEM) in fuel cell application. Initially sulfonated aromatic poly(ether amide)s were designed to be prepared. Although some of them showed promising proton conductivity, comparable to that of Nafion at $80^{\circ} \mathrm{C}$ and $100 \%$ relative humidity, their stability in water became an issue when ion exchange capacity (IEC) was increased to improved the proton conductivity further.[1] To improve proton conductivity of the membrane further while preventing excessive water uptake, synthetic efforts to incorporate fluorinated moiety along the polymer chains have been taken.

\section{Results}

Polycondensation reactions of various diamines, terephthalic acid (TA), and sulfonated terephthalic acid (STA) produced high-molecular-weight sulfonated aromatic poly(ether amide)s (Figure 1; DA-SCPA-xx (Na), where xx indicates the mol\% of STA in the polymer). By 
changing the ratio of TA and STA, sulfonated polymers with different degrees of sulfonation could be obtained. Based on intrinsic viscosity measurement results, all sulfonated polymers were found to have high molecular weights (intrinsic viscosity $>1.0 \mathrm{dL} / \mathrm{g}$ ).

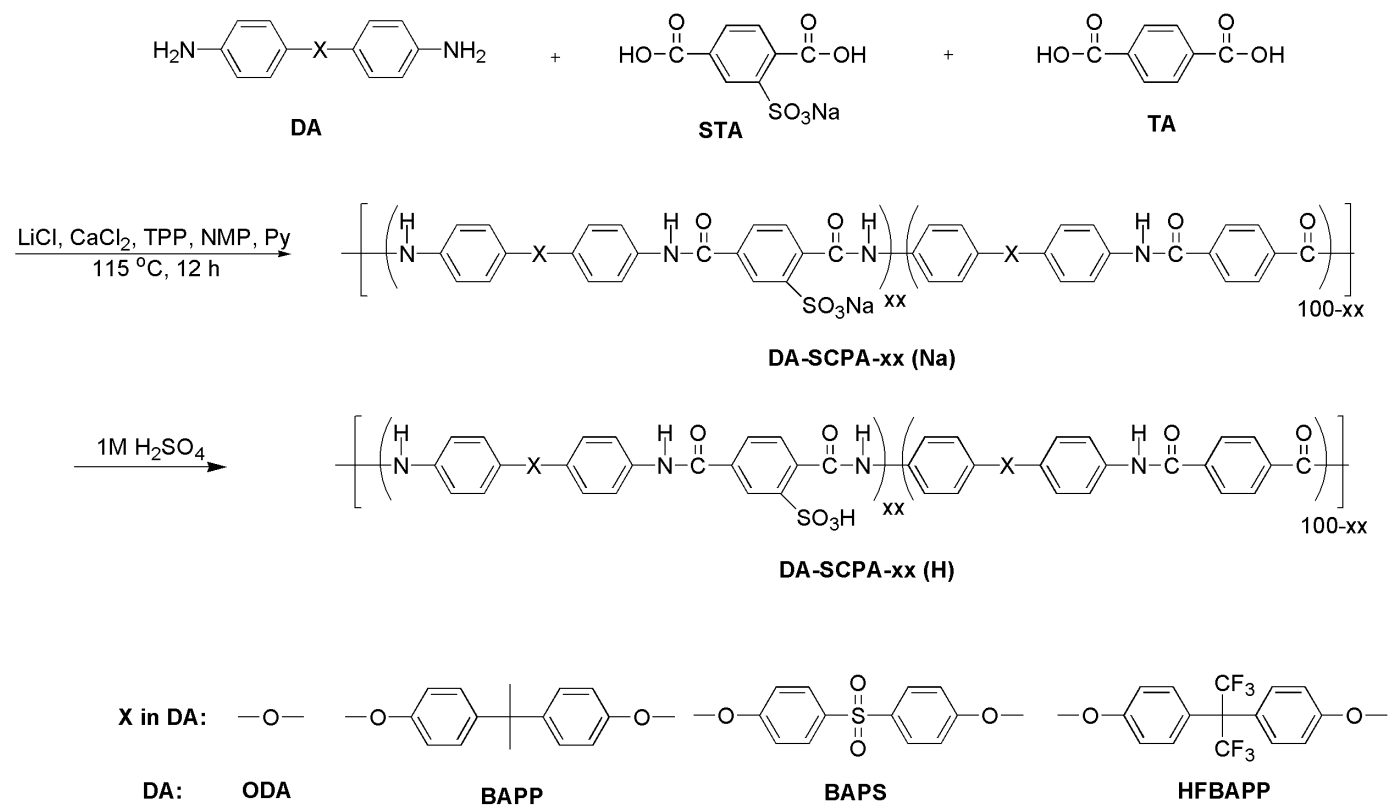

Figure 1. Synthesis of sulfonated aromatic poly(ether amide)s.

Thin film of DA-SCPA ( $\mathrm{Na}$ ) was prepared and conversion of the ionomers to $\mathrm{SO}_{3} \mathrm{H}$ form was conducted in aqueous sulfuric acid solution. Membrane properties of DA-SCPA $(\mathrm{H})$ were evaluated. As can be seen from the results of Table 1, the experimental IEC values measured from the titration agree well with the calculated ones of the theoretical polymer structures that were derived from the molar ratio of TA to STA. This result clearly indicates that the targeted polymer structures were successfully synthesized and all sodium sulfonate form of the sulfonated polymers was quantitatively converted to the corresponding sulfonic acid form. The sulfonic acid form membranes with IEC $=1.00-1.80$ mequiv/g show water uptake values in the range of 1032 weight $\%$, which are relatively lower compared with those of other hydrocarbon based proton exchange membranes having similar IECs. To evaluate oxidative (chemical) stability in a condition similar to fuel cell environment, Fenton test was conducted by soaking the membranes in Fenton's reagent $\left(3 \% \mathrm{H}_{2} \mathrm{O}_{2}\right.$ containing $2 \mathrm{ppm}$ of $\left.\mathrm{FeSO}_{4}\right)$. The oxidative stability was evaluated by measuring the amount time for the membranes to become brittle $\left(\mathrm{T}_{1}\right)$ and to start to dissolve into the reagent $\left(\mathrm{T}_{2}\right)$.

Table 1. Ionic exchange capacity (IEC), water uptake, oxidative stability of sulfonated aromatic poly(ether amide)s.

\begin{tabular}{|c|c|c|c|c|c|}
\hline \multirow{2}{*}{ Sulfonated polymers } & \multicolumn{2}{|c|}{$\begin{array}{c}\text { IEC } \\
\text { (mequiv/g) }\end{array}$} & \multirow{2}{*}{$\begin{array}{c}\text { Water } \\
\text { uptake }(\%)^{\mathrm{d}}\end{array}$} & \multicolumn{2}{|c|}{ Oxidative stability $^{\mathrm{e}}$} \\
\hline & $\operatorname{expt}^{\mathrm{a}}$ & calcd $^{b}$ & & $\mathrm{~T}_{1}(\mathrm{~min})$ & $\mathrm{T}_{2}(\mathrm{~min})$ \\
\hline ODA-SCPA-40 & 1.10 & 1.05 & 17 & 50 & 110 \\
\hline ODA-SCPA-50 & 1.34 & 1.33 & 23 & 45 & 100 \\
\hline ODA-SCPA-60 & 1.58 & 1.56 & 24 & 45 & 95 \\
\hline ODA-SCPA-70 & 1.80 & 1.83 & 33 & 55 & 120 \\
\hline
\end{tabular}




$\begin{array}{cccccc}\text { BAPP-SCPA-70 } & 1.17 & 1.06 & 17 & 65 & 150 \\ \text { BAPS-SCPA-70 } & 1.13 & 1.11 & 10 & 70 & 165 \\ \text { HFBAPP-SCPA-70 } & 0.99 & 0.94 & 13 & 135 & 260\end{array}$

${ }^{a}$ Experimental IEC measured by titration. ${ }^{b}$ Calculated IEC based on structures of sulfonated polyamides. ${ }^{\mathrm{C}}$ Calculated equivalent weight based on structures of sulfonated polyamides. ${ }^{\mathrm{d}}$

Water uptake $(\%)=\frac{W \text { wet }-W \text { dry }}{W \text { dry }} * 100 \%$, where, $W_{\text {dry }}$ and $W_{\text {wet }}$ are the weights of dried and wet membranes, respectively. ${ }^{\mathrm{e}}$ Oxidative stability measured by Fenton test where $\mathrm{T}_{1}$ is the elapsed time for the membrane to start to become brittle and $T_{2}$ is the time for the membrane to start to dissolve in the solution.

Thermal stability of the sulfonated poly(ether amide)s in sulfonic acid form was measured with thermo gravimetric analysis (TGA) in an inert gas atmosphere. The TGA curves of ODA containing polymers are shown in Figure 2 (a). When compared with the thermal stability of non-sulfonated polyamide [ODA-SCPA-0 of Figure 2 (a)], prepared from polycondensation reaction of TA and ODA, the incorporation of sulfonic acid groups into the polymer main chain induced decreased thermal stability of the sulfonated polymer. The first degradation step of the ODA based sulfonated polymers was observed around $300{ }^{\circ} \mathrm{C}$ because of the cleavage of $\mathrm{C}-\mathrm{S}$ bond and the desulfonation of $\mathrm{SO}_{3} \mathrm{H}$. Among the series of ODA based polymers, as the DS increases, the weight loss of the membrane resulting from the desulfonation increases [Figure 2 (a)]. Thermal stabilities of DA-SCPAs derived from different diamine monomers were also studied. As shown in Figure 2 (b), when the four different DA-SCPAs with the same $70 \%$ DS were examined, the dependence of thermal stability on the diamine monomer structure was observed. Similar to the oxidative stability data, HFBAPP-SCPA-70 exhibited the lowest weight loss in TGA.
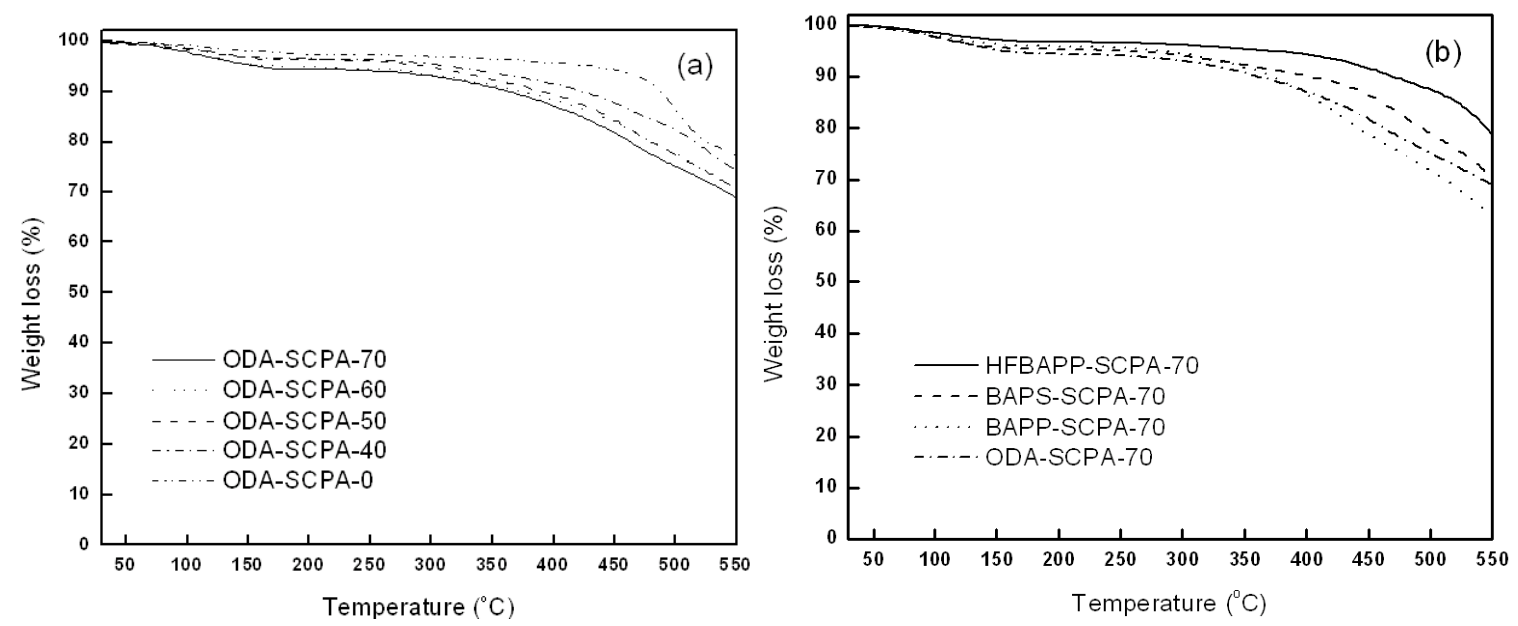

Figure 2. TGA curves of (a) a series of ODA based sulfonated copolyamides in their acid form and (b) a series of $70 \%$ sulfonated copolyamides that were prepared from different diamine monomers.

Proton conductivities of DA-SCPA $(\mathrm{H})$ were measured at various temperatures under $100 \%$ relative humidity using a BekkTech membrane proton conductivity measurement system (Figure 
3). Among membranes tested, ODA-SCPA-70 showed high proton conductivity $(105 \mathrm{mS} / \mathrm{cm})$ at $80{ }^{\circ} \mathrm{C}$, which was comparable to that of Nafion $(104 \mathrm{mS} / \mathrm{cm})$.

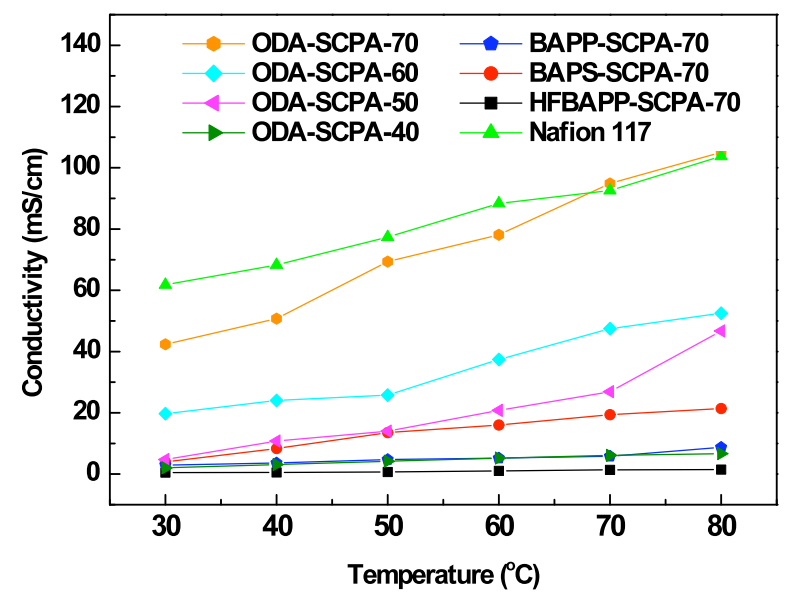

Figure 3. Proton conductivity of sulfonated copolyamides at different temperatures under $100 \%$ relative humidity.

When higher than $70 \mathrm{~mol} \%$ of STA was introduced into the sulfonated polymers to increase IEC and the resulting proton conductivity, the highly sulfonated polymer became unstable in water. Thus, we have prepared fluorine substituted sulfonated poly(ether amide)s (ODA-PFSA$\mathrm{xx}$ and ODA-TFIPA-xx of Figure 4, where $\mathrm{xx}$ is the degree of sulfonation) using a similar polycondensation method above and investigated their membrane properties. The fluorine substitution in the sulfonated polymers resulted in a significant reduction of water uptake level compared to that of non-fluorinated sulfonated polymers reported earlier. Even polymers with 90 mol\% sulfonation level (i.e., ODA-PFSA-90 and ODA-TIFF-90 in Figure 4) exhibited acceptable levels of water uptake (30-40 wt $\%$ in Table 2). Accordingly, we were able to incorporate higher concentration of sulfonic acid moiety into the polymer and synthesize PEMs with higher ionic exchange capacity (IEC $=1.7-2.1$ mequiv $/ \mathrm{g}$ ). The fluorine substituted ODAPFSA and ODA-TFIPA series ionomers showed higher conductivity than the non-fluorinated polymer, ODA-SCPA-70, and Nafion when measured at 100\% relative humidity and different temperature (see Figure 5). The highest conductivity was obtained from ODA-TFIPA-90 (207 $\mathrm{mS} / \mathrm{cm}$ at $\left.80{ }^{\circ} \mathrm{C}\right)$.

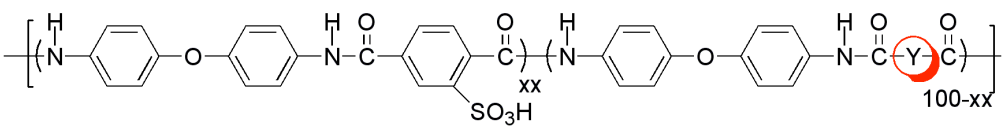

ODA-PFSA-xx, ODA-TFIPA-xx, ODA-SCPA-xx

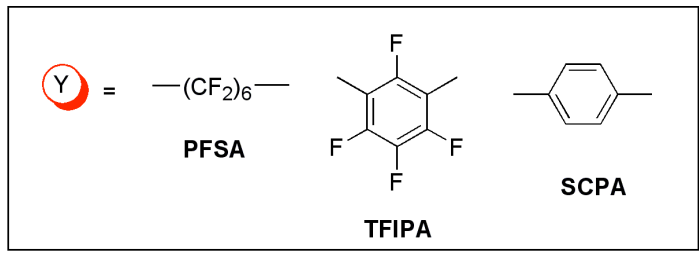

Figure 4. Synthesis of fluorinated sulfonated poly(ether amide)s. 
Table 2. Membrane properties of fluorine substituted sulfonated poly(ether amide)s.

\begin{tabular}{ccccccc}
\hline \multirow{2}{*}{ Sulfonated Polymer } & \multirow{2}{*}{$\begin{array}{c}\text { Thickness } \\
(\mu \mathrm{m})\end{array}$} & IV $(\mathrm{dl} / \mathrm{g})^{\mathrm{a}}$ & $\begin{array}{c}\text { Water } \\
\text { Uptake } \\
(\%)^{\mathrm{b}}\end{array}$ & \multicolumn{2}{c}{ IEC (mequiv/g) } \\
\cline { 5 - 7 } & & & $\operatorname{Exp}^{\mathrm{c}}$ & Calc $^{\mathrm{d}}$ \\
\hline ODA-PFSA-80 & 55 & 1.24 & 8 & 1.74 & 1.72 \\
ODA-PFSA-90 & 50 & 1.50 & 31 & 1.99 & 2.00 \\
ODA-TFIPA-70 & 50 & 1.45 & 20 & 1.61 & 1.65 \\
ODA-TFIPA-80 & 85 & 1.37 & 39 & 1.81 & 1.87 \\
ODA-TFIPA-90 & 50 & 1.35 & 41 & 2.05 & 2.09 \\
\hline
\end{tabular}

${ }^{a}$ Intrinsic viscosity measured from N,N-dimethylacetamide solution with $0.1 \mathrm{M} \mathrm{NaI}$.

${ }^{\mathrm{b}}$ Water uptake $=$ weight of wet membrane/weight of dry membrane $\mathrm{x} 100$.

${ }^{c}$ Experimental ionic exchange capacity measured by titration.

${ }^{d}$ Theoretically calculated ionic exchange capacity estimated from the molar ratio of sulfonated and non-sulfonated monomers.

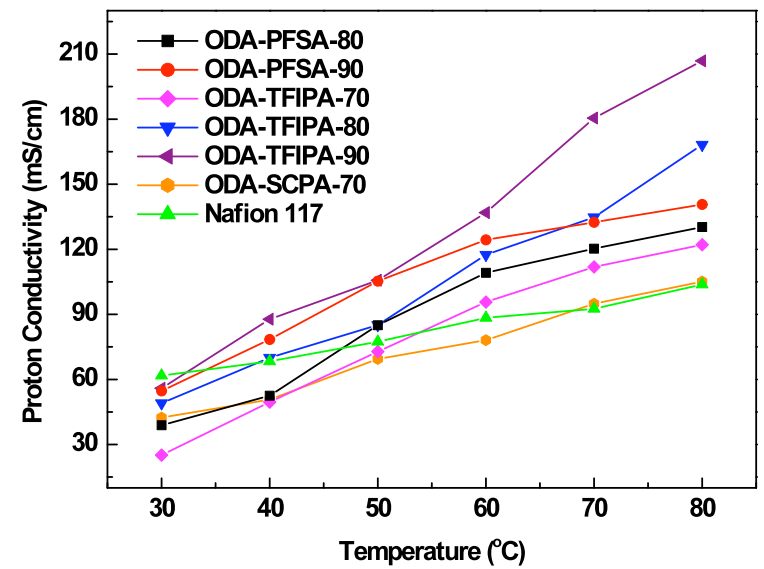

Figure 5. Proton conductivities of fluorine substituted sulfonated poly(ether amide)s and Nafion at $100 \%$ relative humidity and different temperature.

\section{Conclusions and Future Directions}

(A) Highly proton conductive fluorine containing sulfonated PEMs are developed

(B) Regarding to future direction, durability test of the new developed PEMs in membrane electrode assembly (MEA) and incorporation of fluorine substituents into sulfonic acid moiety instead of polymer main chain are suggested.

\section{Special Recognitions \& Awards/Patents Issued}

\section{Publications/Presentations}


1. T. S. Jo, C. H. Ozawa, B. R. Eagar, L. V. Brownell, D. Han, C. Bae, "Synthesis of Sulfonated Aromatic Poly(ether amide)s and Their Application to Proton Exchange Membrane Fuel Cells", J. Polym. Sci., Part A: Polym. Chem. 2009, 47, 485-496.

2. T. S. Jo, C. Bae "Synthesis of Ionic Conducting Polymers for Fuel Cell Applications and Biodiesel Productions" $42^{\text {nd }}$ Western Regional Meeting of the American Chemical Society, Las Vegas, NV, September 23-27, 2008.

3. C. Bae "New Functional Polymers for Alternative Energy Application" UNLV Renewable Energy Symposium, Las Vegas, NV, August 20, 2008.

4. E. Kim, P. Weck, N. Balakrishnan, C. Bae, "Nanoscale Building Blocks for the Development of Novel Proton Exchange membrane Fuel Cells", J. Phys. Chem. B. 2008, 112, 3283-3286

\section{References}

1. T. S. Jo, C. H. Ozawa, B. R. Eagar, L. V. Brownell, D. Han, C. Bae, "Synthesis of Sulfonated Aromatic Poly(ether amide)s and Their Application to Proton Exchange Membrane Fuel Cells", J. Polym. Sci., Part A: Polym. Chem. 2009, 47, 485-496.

\section{Acronyms}

PEM Polymer electrolyte membrane

IEC Ionic exchange capacity 


\section{ATTACHMENT G}

\section{P.I.: Tao Pang}

Project: Quantum Monte Carlo simulation of hydrogen in a host matrix We have reproduced the simulation results for a hydrogen molecule in a spheroid box. We have also carried out preliminary simulation results for a hydrogen molecule in a double-sphere box with its volume fixed with that of the corresponding optimized spheroid box under the same bond length. Our results show that a hydrogen molecule has a higher energy in a double-sphere box. This means that a hydrogen molecule stored in a matrix with a cavity similar to a doublesphere box is easier to be extracted.

Dr. Eunja Kim, Dr. Philippe F. Weck, and I have submitted a proposal to the DOE for continuing our study on the platinum surface and other catalysts in hydrogen fuel cells after the FCAST program.

Dr. Hongmei Wang developed simulation that calculates pair-correlation function and structure factor of a uniform quantum system. Periodic boundary condition has been implemented in the simulation in dealing with a bulk material and the Fourier transform with Filon's integration formula is adopted in constructing the structure factor.

Dr. Hongmei Wang has stabilized the program for calculating radial distribution function and structure factor of the liquid and solid phases of a uniform quantum system. She has also introduced a guiding wave function for a quantum solid and found the preliminary collective excitations of the system in both the liquid and solid phases.

The most recent work of Dr. Hongmei Wang concentrates on the structure factor and the collective excitations of the solid helium-4. She has found a similar longwavelength limit of the excitations that is similar to the liquid case. The question we are still trying to answer is whether there is a stable roton region in the solid case.

\section{Publications:}

T. Pang, "An Introduction to Computational Physics, 2nd Edition" (Cambridge University Press, Cambridge, UK, 2006), 402 pp.

H. Ma and T. Pang, "Path-integral quantum Monte Carlo study of a mixture of Bose-Einstein Condensates," Physics Letters A 351, 92-96 (2006).

E. Kim, T. Pang, W. Utsumi, V.L. Solozhenko, and Y. Zhao, "Cubic phases of BC2N: A first-principles study," Physical Review B 75, 184115 (2007).

\section{Presentations/Posters:}

E. Kim and T. Pang, "First-principles study of the oxygen-reduction reaction on the $\operatorname{Pt}(100)$ surface," American Physical Society Annual Meeting, New Orleans, Louisiana, March 10-14, 2008. 


\section{ATTACHMENT H}

PI: Eunja Kim

\section{Objectives:}

- Design materials for hydrogen storage and fuel cell application;

- Quantify reaction barrier and hydrogen adsorption/dissociation energies;

- Understand physical/chemical changes under various conditions such as pressure and metallic doping;

- Optimize hydrogen wt \% for hydrogen storage applications and operating temperatures and durability of hydrogen fuel cell membranes.

\section{Technical Barriers:}

The technical barriers in current hydrogen storage and fuel cell membrane research are: Hydrogen Storage:

(i) $\mathrm{H}$ Weight \% and Volumetric Weight to meet DOE Target ;

(ii) Lack of understanding of $\mathrm{H}$ physisorption/chemisorption;

(iii) Recycling hydrogenation/dehydrogenation processes.

Fuel cell membrane: Durability at high temperature.

\section{Technical Targets}

- Development of carbon nanostructures tailored for hydrogen storage;

- Development of high temperature polymer membranes for the fuel cells.

\section{Accomplishments}

- Designed carbon 3D-nanoframeworks tailored for hydrogen storage using density functional theory based calculations;

- Investigated hydrogenation of single-wall carbon nanotubes using polyamine reagents in collaboration with experimental study;

- Verified the mechanism of fullerene hydrogenation by polyamines;

- Examined successive adsorption of hydrogen atoms on a sp2-bonded boron nitride graphitic sheet;

- Demonstrated phase changes of metal hydrides ( $\mathrm{MXH4}$ : $\mathrm{M}=\mathrm{Na}, \mathrm{K}, \mathrm{X}=\mathrm{Al}, \mathrm{B}$ ) under pressure;

- Designed nano-scale building blocks for the development of novel proton exchange membrane fuel cells;

- Presented research outcomes at scientific meetings and conferences;

- Published 14 papers in high-impact journals, producing 2 cover articles as shown below. 

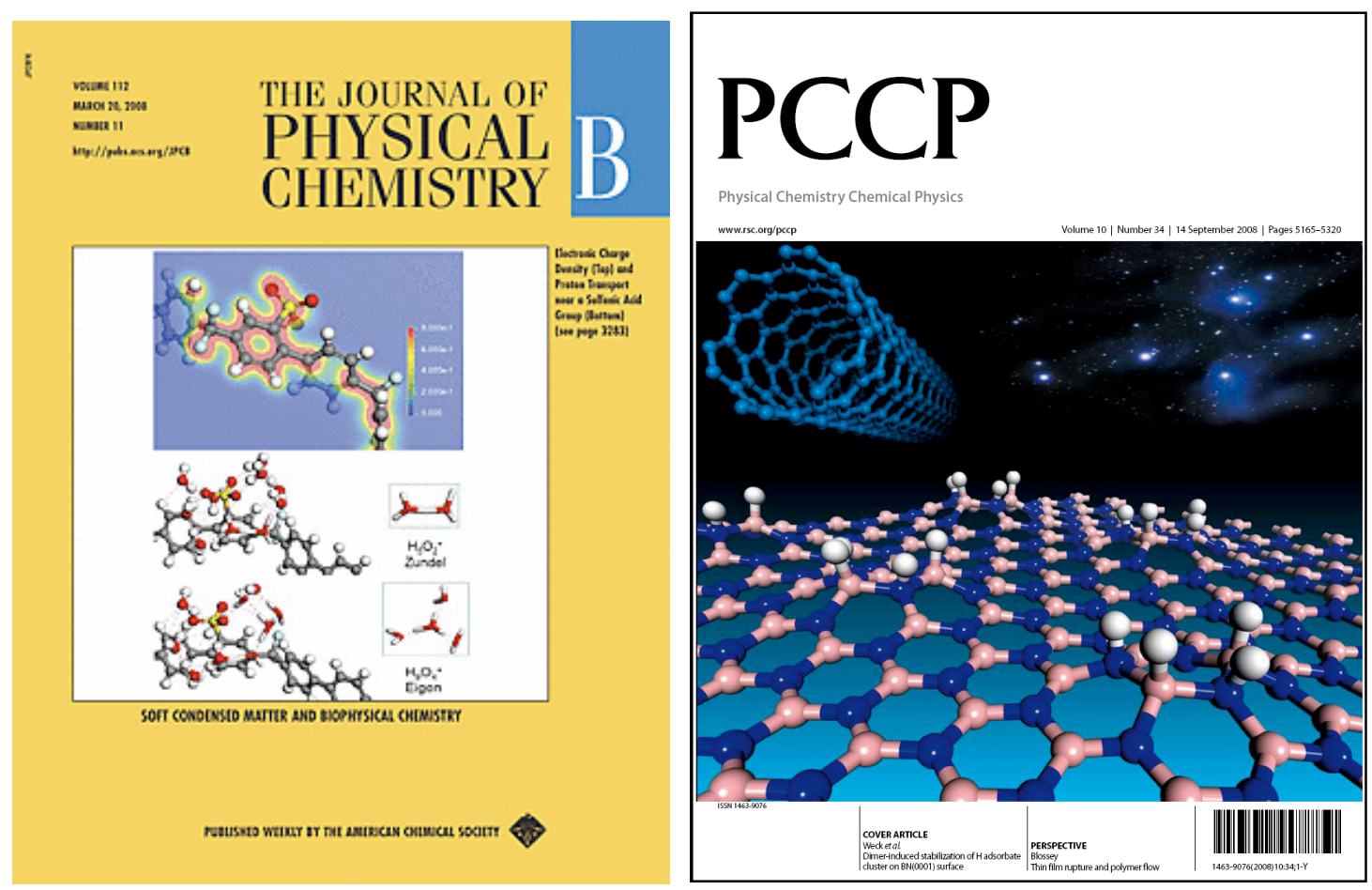

\section{Results:}

My theoretical research effort has been focused on development of materials suitable for hydrogen storage and fuel cell membranes as discussed below. Main collaborators involved in this project are Prof. P. Weck (UNLV), Prof. B. Yakobson (Rice), Prof. N. Balakrishnan (UNLV), Prof. D. Tomanek, and Prof. C. Bae (UNLV).

\section{(1) Design and characterization of carbon nanostructures tailored for hydrogen storage}

\section{(i) Designing carbon nanoframeworks tailored for hydrogen storage:}

Based on first-principles calculations, we propose a novel class of 3-D materials consisting of small diameter single-walled carbon nanotubes (SWCNTs) functionalized by organic ligands as potential hydrogen storage media. Specifically, we have carried out density functional theory calculations to determine the stable structures and properties of nanoframeworks consisting of $(5,0)$ and $(3,3)$ SWCNTs constrained by phenyl spacers. Valence and conduction properties, as well as normal modes, of pristine nanotubes are found to change significantly upon functionalization, in a way that can serve as experimental diagnostics of the successful synthesis of the proposed framework structures. Ab initio molecular dynamics simulations indicate that such systems are thermodynamically stable for onboard hydrogen storage. 

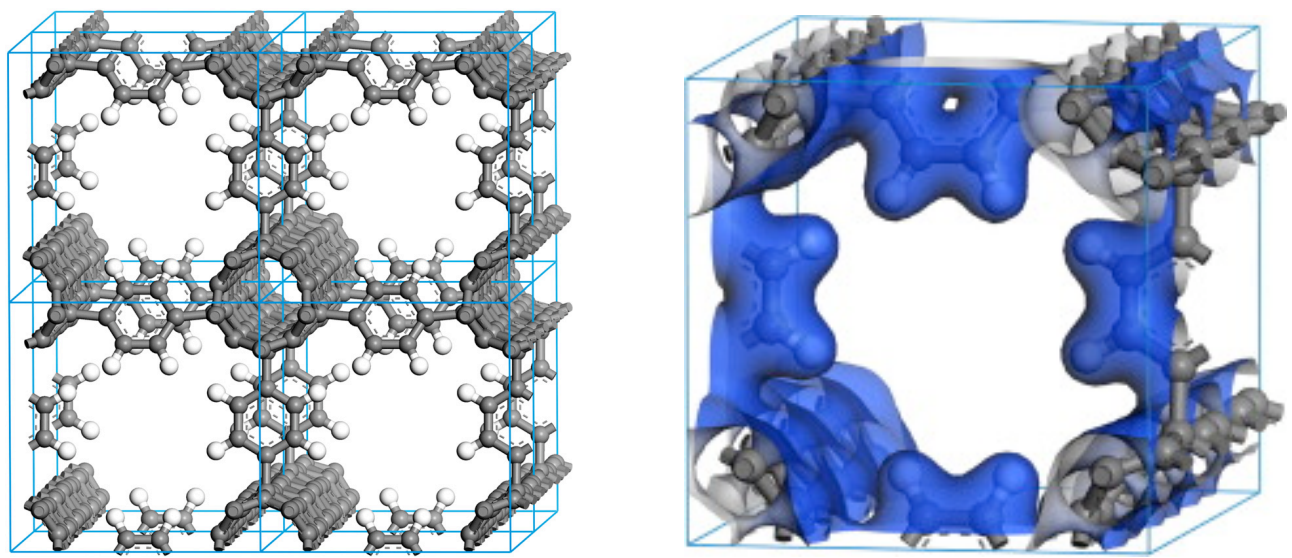

Fig. 1. Optimized nanoframework structure (left) and its electronic charge density (right) consisting of $(5,0)$ SWCNTs constrained by phenyl spacers. The unit cell is shown in blue.

Further reading is available at G. Miller, J. Kintigh, E. Kim et al., J. Am. Chem. Soc. 1302296 (2008).

\section{(ii) Hydrogenation of Single-Wall Carbon Nanotubes Using Polyamine Reagents: Combined Experimental and Theoretical Study}

We combine experimental observations with ab initio calculations to study the reversible hydrogenation of single-wall carbon nanotubes using high boiling polyamines as hydrogenation reagents. Our calculations characterize the nature of the adsorption bond and identify preferential adsorption geometries at different coverages. We find the barrier for sigmatropic rearrangement of chemisorbed hydrogen atoms to be about $1 \mathrm{eV}$, thus facilitating surface diffusion and formation of energetically favored, axially aligned adsorbate chains. Chemisorbed hydrogen modifies the structure and stability of nanotubes significantly and increases the inter-tube distance, thus explaining the improved dispersability in solvents like methanol, ethanol, chloroform, and benzene.
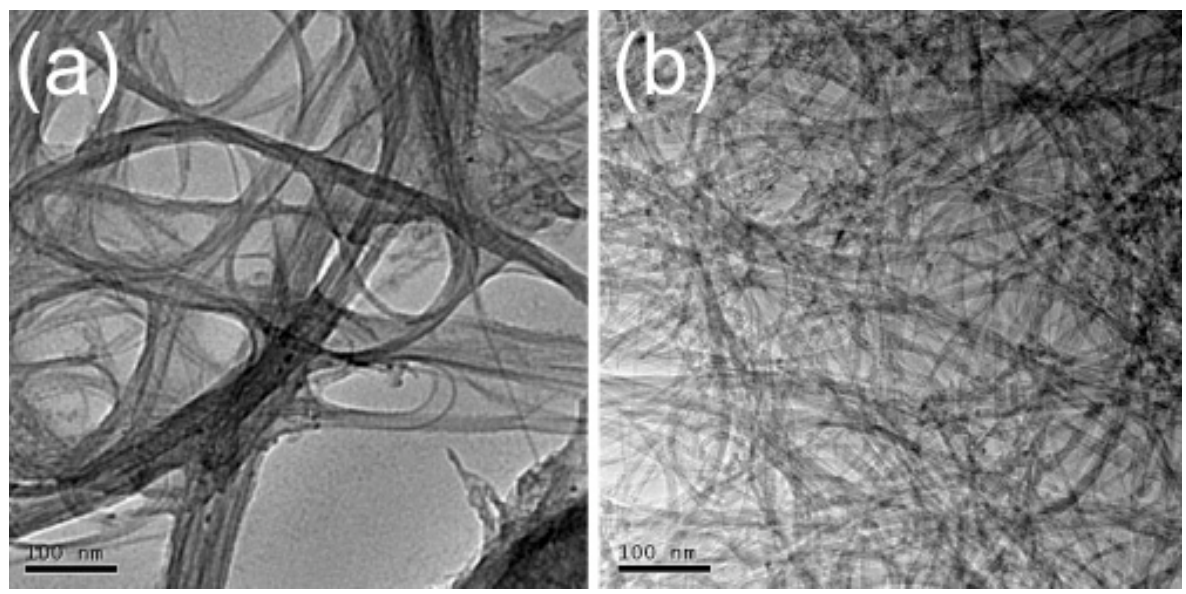

Fig. 2. Transmission Electron Microscope (TEM) images of (a) bundled SWNTs before hydrogenation and (b) debundled H-SWNTs after polyamine hydrogenation. 
Further reading is available at G. Miller, J. Kintigh, E. Kim et al., J. Am. Chem. Soc. 1302296 (2008).

(iii) Mechanism of fullerene hydrogenation by polyamines:

We use ab initio density functional calculations to study the microscopic mechanism underlying the recently demonstrated hydrogenation of the $\mathrm{C}_{60}$ fullerene by diethylenetriamine reagent. Our results indicate that the optimal monoaddition reaction is exothermic, involving an about $0.5 \mathrm{eV}$ high activation barrier associated with the simultaneous docking of the polyamine functional group and $\mathrm{H}$ transfer to $\mathrm{C}_{60}$. Our calculated vibrational frequencies can be used to experimentally confirm the presence of hydroamination adducts as a necessary prerequisite for successful hydrogenation of $\mathrm{C}_{60}$.
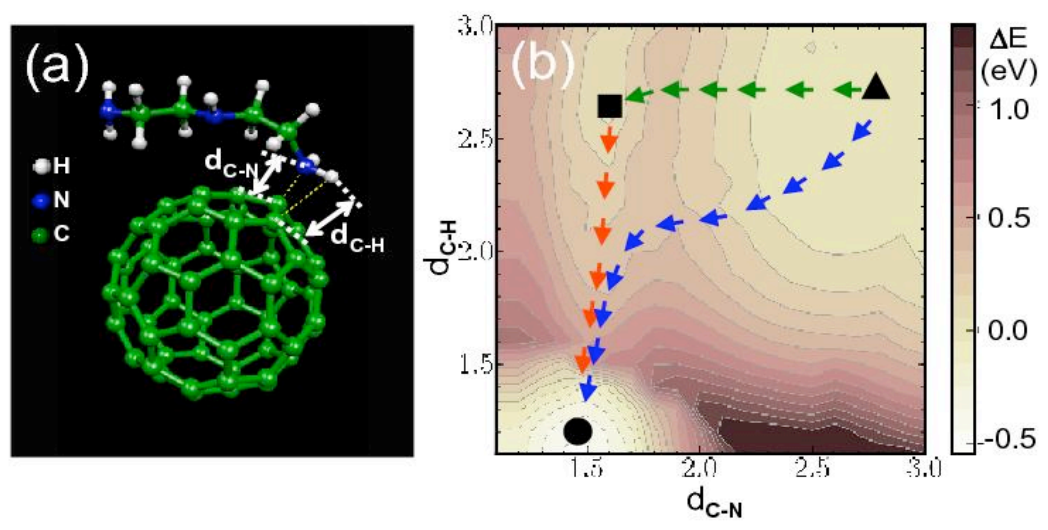

Fig. 3 (a) Schematic of the DETA-C60 geometry associated with the simultaneous docking and H transfer process and (b) Potential energy surface of the DETA-C60 complex .

Further reading is available at E. Kim et al., Phys. Rev. B. 78, 113404 (2008).

(iv) Dimer-induced stabilization of $\mathrm{H}$ adsorbate cluster on $\mathrm{BN}(0001)$ surface:

Using first-principles calculations, we have studied successive adsorption of hydrogen atoms on a $s p^{2}$-bonded boron nitride graphitic sheet. Our calculations show that clustering proceeds through the creation of contiguous $\mathrm{H}-\mathrm{H}$ orthodimer structures stabilizing the $\mathrm{H}$ adsorbate cluster on the $\mathrm{BN}(0001)$ surface, leading eventually to the formation of hydrogen-contiguous boat-shaped quartets.
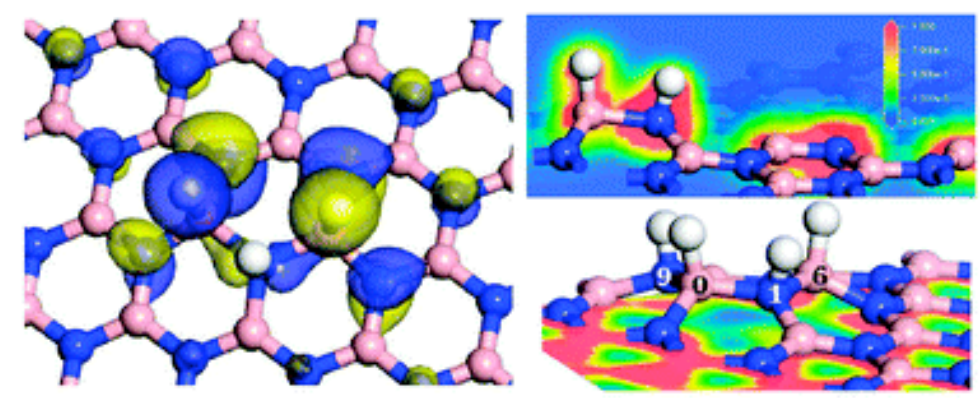
Fig. 4 Highest occupied molecular orbital (HOMO) of $\mathrm{H}$ triplet (left) and electronic charge density cross section of the most stable $\mathrm{H}-\mathrm{H}$ pair (right-top) and $\mathrm{H}$ quartet (right-bottom) adsorbed on BN(0001).

Further reading is available at P. Weck and E. Kim et al., Phys. Chem. Chem. Phys. 10 (Communication), 5184 (2008). [September 14, 2008 Cover Page].

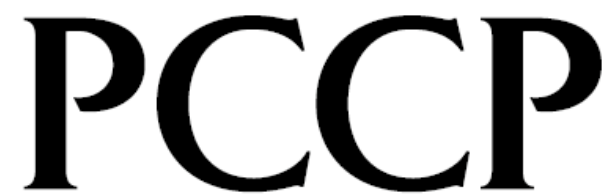

Physical Chemistry Chemical Physics

www.rsc.org/pecp

Volume 10 | Number 34 | 14 September 2008 | Pages 5165-5320

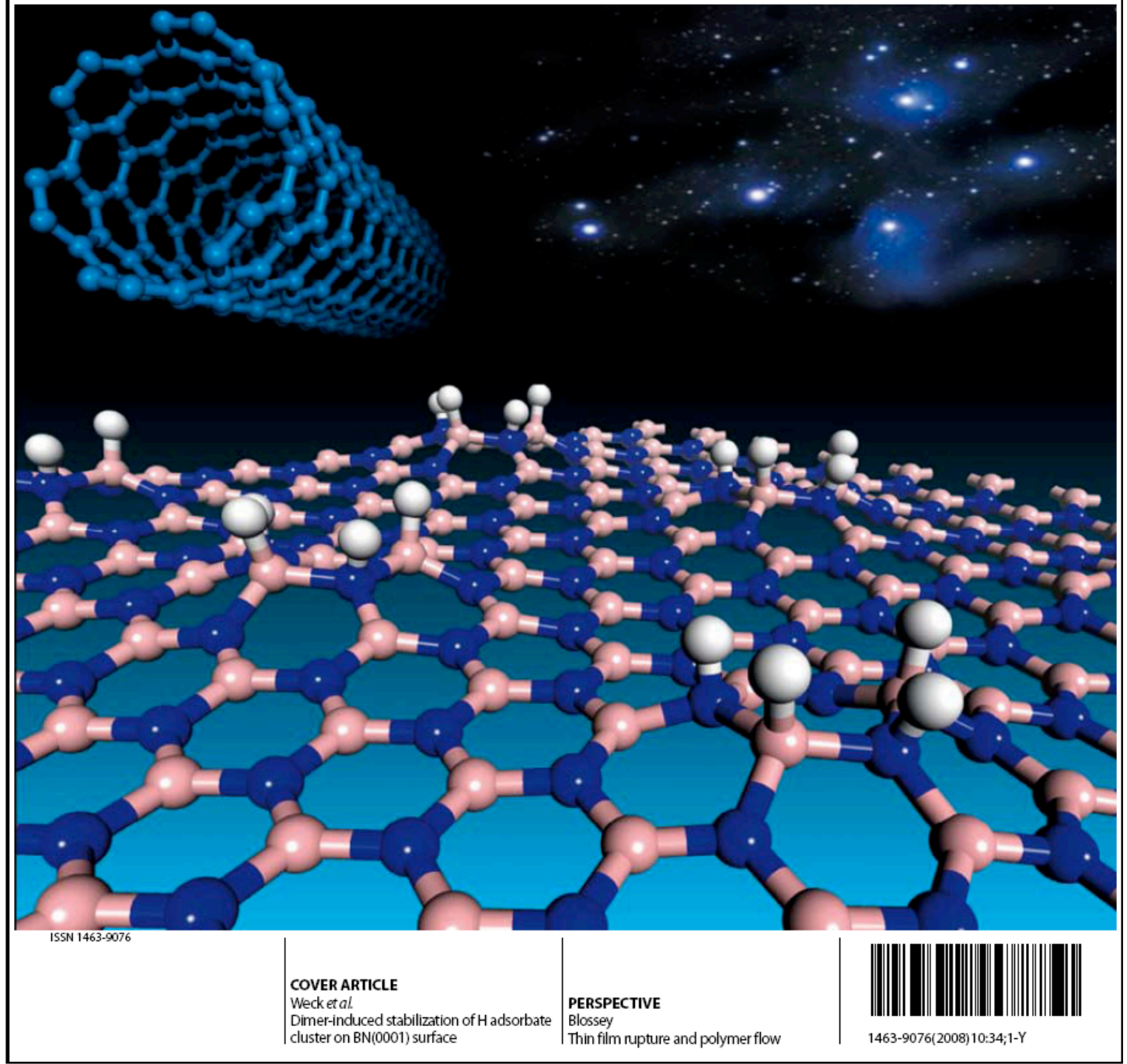


(2) Development of metal hydrides for hydrogen storage materials : $\mathrm{MXH}_{4}(\mathrm{M}=$ $\underline{\mathrm{Li}, \mathrm{Na}, \mathrm{X}=\mathrm{Al}, \mathrm{B})}$

Our collaborative efforts between theory and experiments to seek materials suitable for hydrogen storage have been extended to complex metal-hydrides such as $\mathrm{NaBH}_{4}$, $\mathrm{NaAlH}_{4}$, and $\mathrm{KBH}_{4}$. Structural characterization performed experimentally and theoretically shows that alkali-borohydrides such as $\mathrm{NaBH}_{4}$ and $\mathrm{KBH}_{4}$ tend to follow the generic behavior in pressure induced structural changes. They undergo transitions from the cubic phase to the tetragonal phase, followed by another transition to the orthorhombic phase, while $\mathrm{NaAlH}_{4}$ undergoes a structural transition from tetragonal to monoclinic phases under pressure. High pressure phase was observed by x-ray diffraction and Raman scattering measurements and confirmed by ab initio calculations using the local density approximation (LDA) and the generalized gradient approximation (GGA). Structural studies at high pressure are necessary and important to understand the synthesized new compounds using ball-milling methods to enhance the dehydrogenation process. A collaborative effort between theory and experiment enable us to predict the structural stability and to understand the phase diagram of this class of compounds, which will help us to tune the properties suitable for storing/releasing hydrogen in the system whenever it is necessary. Three manuscripts are published in peer-reviewed journals.
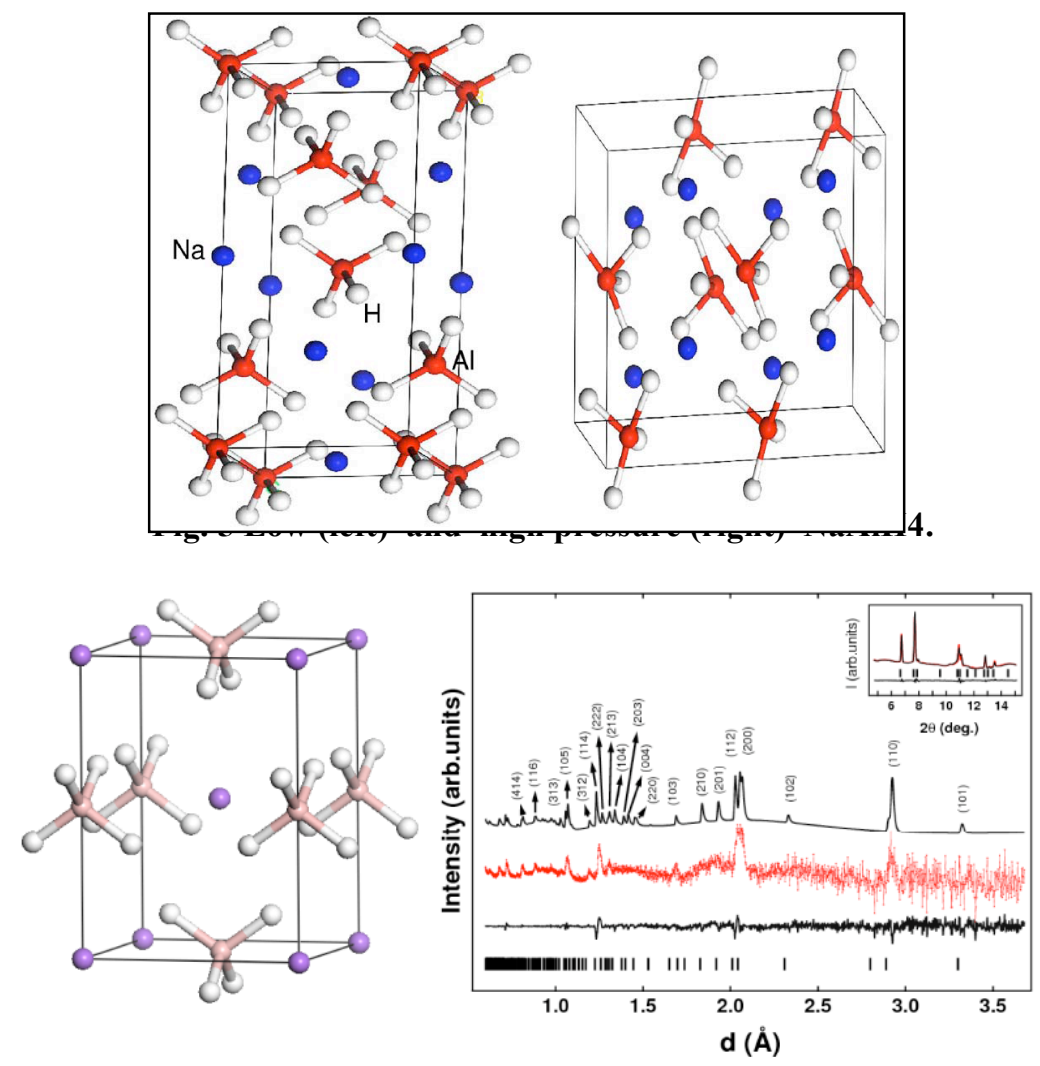

Fig. 6 The intermediate tetragonal phase: Crystalline structure (left) and neutron diffraction pattern (right). 
Further reading is available at E. Kim et al., J. Phys. Chem. B 111 (Letter), 13873 (2007).

\section{(3) Nanoscale Building Blocks for the Development of Novel Proton Exchange Membrane Fuel Cells}

Developing efficient, reliable, environmentally friendly energy sources is one of the most challenging tasks of the 21 st century. Among available alternative energy options, fuel cells are recognized as an ideal energy solution for many applications, including transportation and portable electronics. The proton exchange membrane (PEM), which separates the fuel from the oxidant but allows for proton transport from the anode to the cathode, is the most important component of PEMFCs. We propose a new type of sulfonated aromatic polyarylenes as candidate building blocks for proton exchange membranes. Density functional theory calculations and ab initio molecular dynamics simulations suggest that desulfonation is limited at high temperatures, owing to the strong aryl- $\mathrm{SO}_{3} \mathrm{H}$ bond induced by the electron-deficient aromatic ring, and that the proposed polymers exhibit good thermomechanical stability due to the robust aromatic main-chain repeating unit. Simulations also emphasize the importance of the Grotthuss-type mechanism, with interconversion between Eigen $\left(\mathrm{H}_{9} \mathrm{O}_{4}{ }^{+}\right)$and $\mathrm{Zundel}$ cations $\left(\mathrm{H}_{5} \mathrm{O}_{2}{ }^{+}\right)$as limiting structures, for the hydrated proton transport in the vicinity of the sulfonic acid groups.

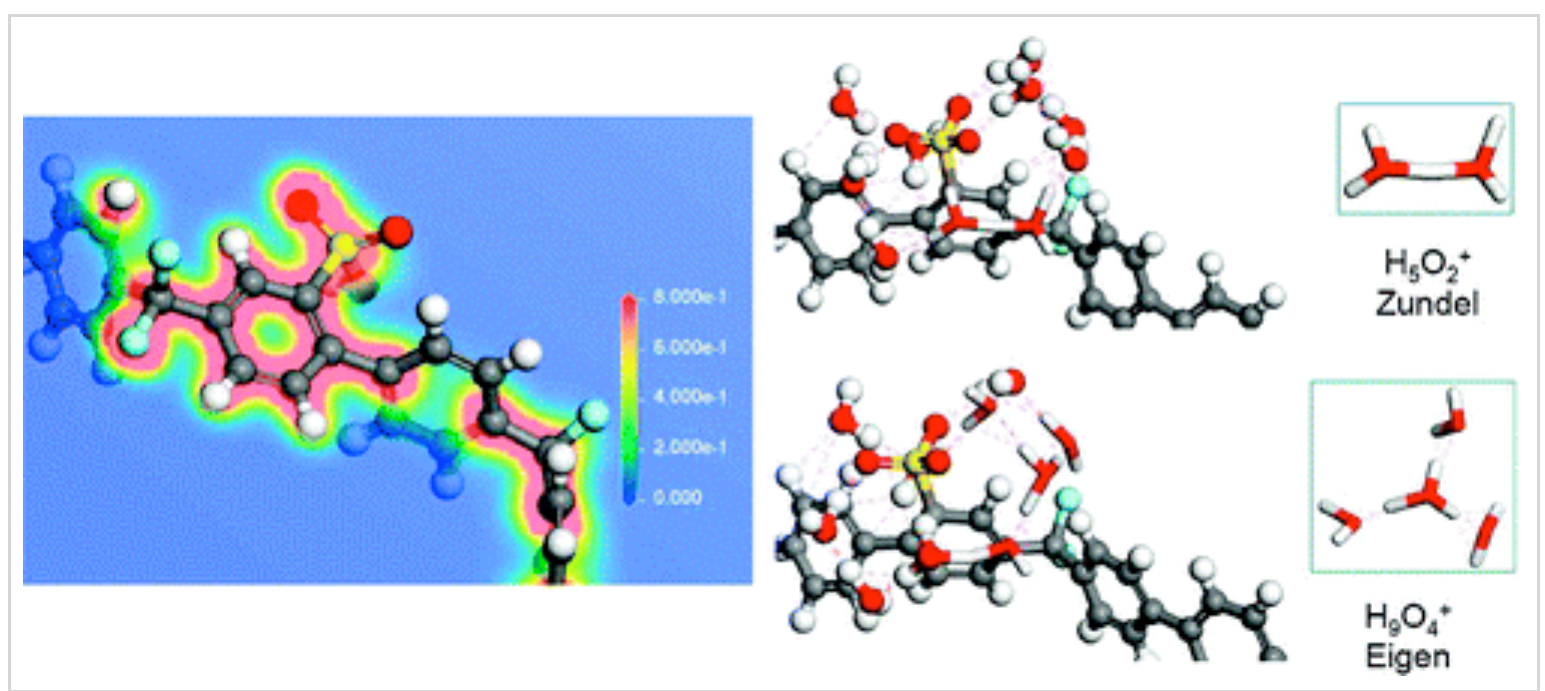

Fig. 7 Projected electronic charge densities of SPA-1 and (b) Snapshots of molecular dynamics simulations showing the proton transport mechanisms in the vicinity of hydrated sulfonic acid protogenic groups of the SPA-1 fragment at $300 \mathrm{~K}$. The snapshots correspond to (a) $0.1 \mathrm{ps,} \mathrm{(b)} 0.7 \mathrm{ps,}$ and (c) $1.1 \mathrm{ps}$. The dashed lines depict the hydrogen-bonded network of water molecules.

Further reading is available at P. Weck and E. Kim et al., Phys. Chem. Chem. Phys. 10 (Communication), 5184 (2008). [March 20, 2008 Cover Page]. 


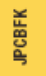

VOLUME 112

MARCH 20, 2008

NUMBER 11

http://pubs.acs.org/JPCB

\section{THE JOURNAL OF PHYSICAL CHEMISTRY}
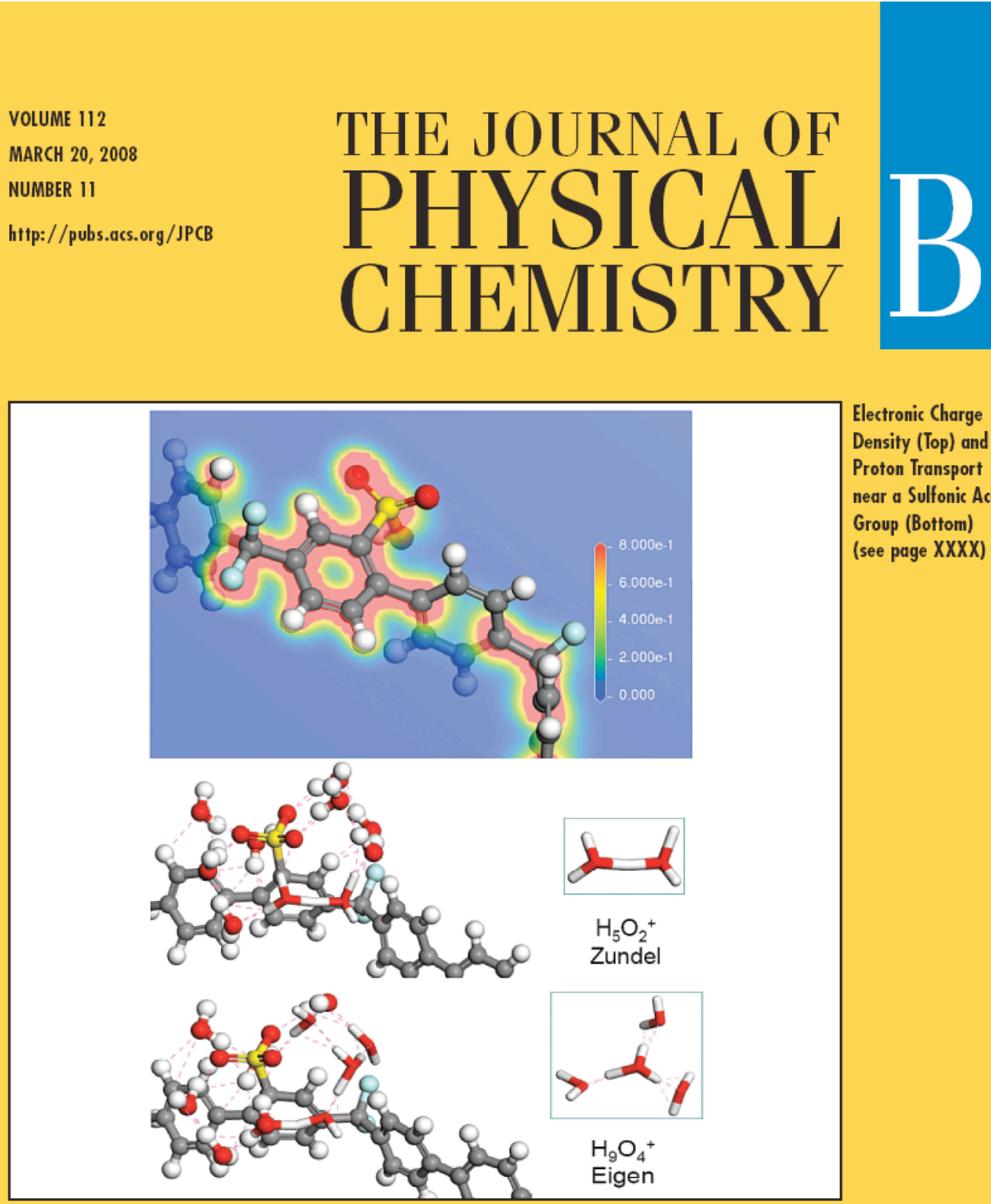

Electronic Charge Density (Top) and Proton Transport near a Sulfonic Acid Group (Bottom) (see page XXXX)

SOFT CONDENSED MATTER AND BIOPHYSICAL CHEMISTRY

PUBLISHED WEEKLY BY THE AMERICAN CHEMICAL SOCIETY 
1. K. Chang, D. Tomanek, E. Kim, P. F. Weck, "Hydrogen storage in ammonia borane: Ab initio study of the de- and rehydrogenation mechanisms", 2009 APS March Meeting, Pittsburgh Denver, CO, March 16-20, 2009.

2. E. Romano, S. Tkachev, M. Pravica, E. Kim, and P. Weck, "Raman spectrum of 1,3,5,7-cyclooctatetraene as a function of pressure", 2009 APS March Meeting, California Section, Cal. State University, CA, October 17-18, 2008.

3. E. Kim, R. Kumar, A. Cornelius, M. Nicol, S. Vogel, J. Zhang, M. Hartl, A. Stowe, L. Daemen, and Y. Zhao, "Structural characterization of sodium borohydrides: Theory and Experiments”, International Symposium on Materials Issues in a Hydrogen Economy, November 12-15 (2007), Richmond, VA.

4. R. Kumar, E. Kim, and A. Cornelius, "Structural changes in RbBH4 under pressure investigated by Synchrotron powder X-ray diffraction and theoretical studies", International Symposium on Materials Issues in a Hydrogen Economy, November 12-15 (2007), Richmond, VA.

5. P. Weck, E. Kim, N. Balakrishnan, H. Cheng, and B. Yakobson, "Lithiumdecorated carbon nanoframeworks tailored for hydrogen storage", International Symposium on Materials Issues in a Hydrogen Economy, November 12-15 (2007), Richmond, VA.

6. E. Kim, D. Stucke, and T. Pang, "Oxidation of Pt (100) surface: Ab initio studies", 2007 APS March Meeting, Denver, CO, March 5-9, 2007.

7. E. Kim, R. Kumar, A. Cornelius, N. Micol, S. C. Vogel, J. Zhang, M. Hartl, A. C. Stowe, L. Daemen, and Y. Zhao, "Pressure-induced structural transitions in $\mathrm{NaBH}_{4}$ ", SMEC 2007 meeting, Miami, FL, April 15-20, 2007.

8. H. Giefers, C. Gobin, B. Keifer, E. Kim, Y. Lee, M. Nicol, J. Orwig, M. Pravica, O. Tschauner, B. Yulga, 'X-ray diddraction and spectroscopy at high pressures: Recent studies', SRMS-5 Conference, Chicago, July 30- Aug. 2, 2006.

9. P. F. Weck, E. Kim, N. Balakrishnan, H. Cheng, "Design of tailored carbon nanostructures for hydrogen storage”, 2007 APS March Meeting, Denver, CO, March 5-9, 2007.

10. E. Kim, "Theoretical study of solid sodium borohydrides under Pressure", MRS Fall meeting, Boston, MA, November 26 - 28, 2006.

11. E. Kim, "Instability of carbon nanotubes: Molecular-dynamics Simulations", International Symposium on Materials Issues in Hydrogen Production and Storage, Santa Barbara, CA, August 20-25, 2006.

12. P. F. Weck, T.J. Dhilip Kumar, E. Kim, and N. Balakrishnan, "Computational study of hydrogen storage in organometallic compounds", International Symposium on Materials Issues in Hydrogen Production and Storage, Santa Barbara, CA, August 20$25,2006$. 